\title{
SIMULACIÓN DE MOVIMIENTOS FUERTES DEL TERRENO MEDIANTE FUNCIONES DE GREEN EMPÍRICAS. APLICACIÓN EN EL CÁLCULO SÍSMICO DE ESTRUCTURAS
}

\author{
MÁSTER UNIVERSITARIO DE ESTRUCTURAS
}

CURSO 2011-2012

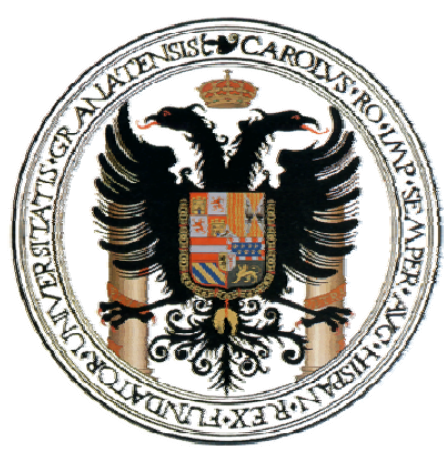

UNIVERSIDAD DE GRANADA

Autor:

Francisco Antonio García Villena 


\title{
SIMULACIÓN DE MOVIMIENTOS FUERTES DEL TERRENO MEDIANTE FUNCIONES DE GREEN EMPÍRICAS. APLICACIÓN EN EL CÁLCULO SÍSMICO DE ESTRUCTURAS
}

\author{
MÁSTER UNIVERSITARIO DE ESTRUCTURAS \\ CURSO 2011-2012
}

DEPARTAMENTO DE MECÁNICA DE ESTRUCTURAS E INGENIERÍA HIDRÁULICA

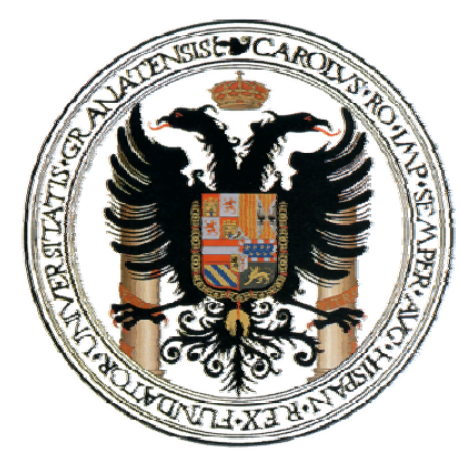

UNIVERSIDAD DE GRANADA

Autor:

Francisco Antonio García Villena

Ingeniero de Caminos, Canales y Puertos

\section{Tutores:}

Dr. Gerardo Alguacil de la Blanca Dr. Francisco Vidal Sánchez

Departamento de Física Teórica y del Cosmos

$e$

Instituto Andaluz de Geofísica

UNIVERSIDAD DE GRANADA 


\section{ÍNDICE}

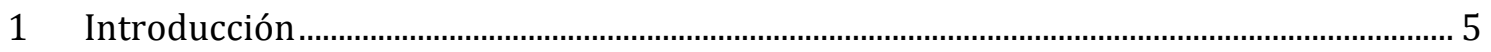

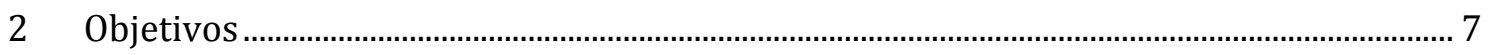

3 Contextualización en el cálculo estructural .......................................................................... 9

3.1 La acción sísmica en el cálculo estructural. ....................................................................... 9

3.1.1 Caracterización mediante espectros elásticos de respuesta. .............................10

3.1.2 Caracterización mediante espectros de input de energía....................................10

3.1.3 Caracterización mediante acelerogramas.............................................................11

3.2 La acción sísmica en el marco normativo.........................................................................13

3.2.1 Aceleración sísmica de cálculo..............................................................................14

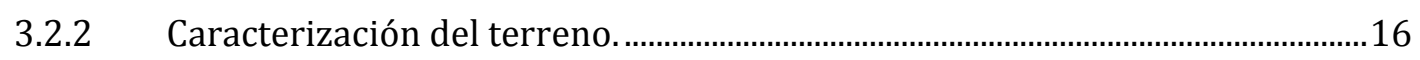

3.2.3 Espectros de respuesta elástica según la NCSE-02 ...............................................17

3.2.4 Espectros de respuesta elástica según la NCSP-07............................................18

4 Simulación de Terremotos. Fundamentos ................................................................................21

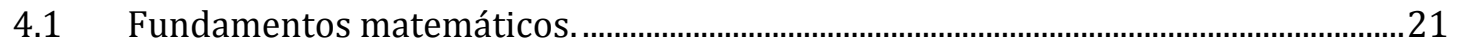

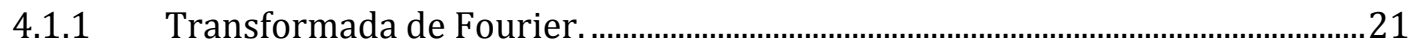

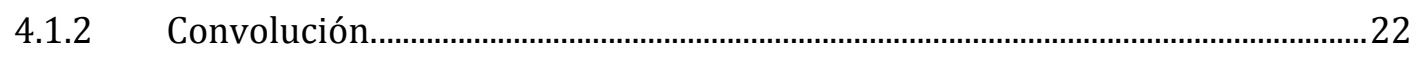

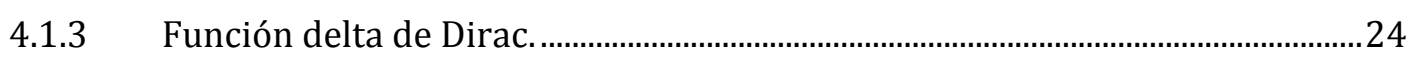

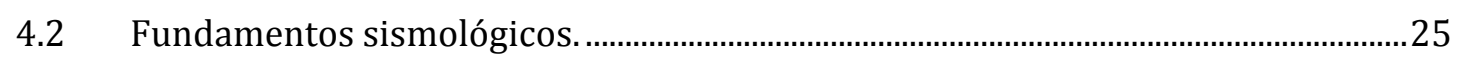

4.2.1 Fallas, Tensor momento y Mecanismo de los terremotos. .................................25

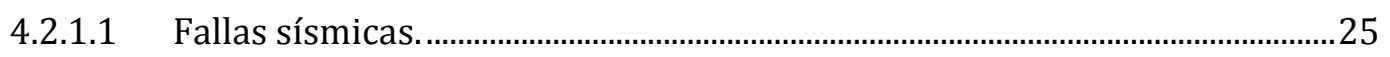

4.2.1.2 Tensor momento y Momento sísmico escalar. ..................................................28

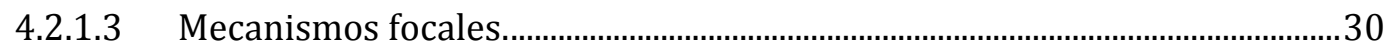

4.2.2 Magnitud de un terremoto................................................................................

4.3 Teorema de representación. La función de Green..........................................................32

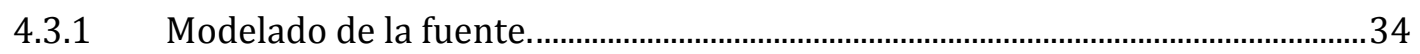

4.3.1.1 Modelo de Haskell. Parámetros.............................................................................. 34

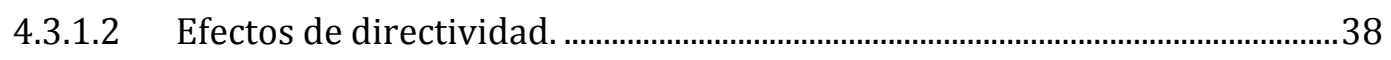

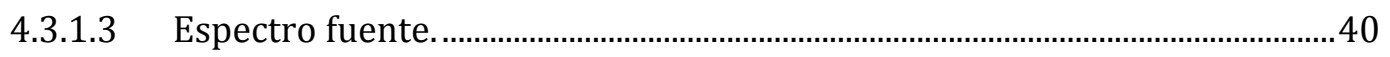

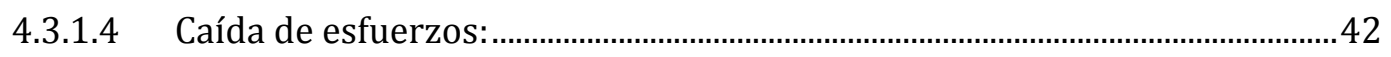

4.4 Enfoque teórico a la simulación de movimientos intensos del terreno.....................44

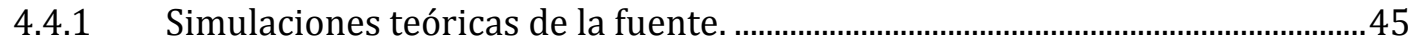

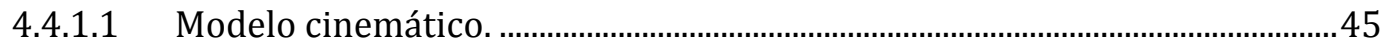

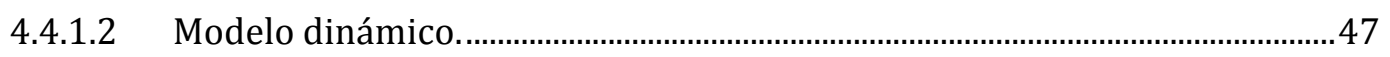


4.4.2 Simulación teórica de los efectos del camino y del sitio.........................................48

4.5 Enfoque semiempírico de la simulación de movimientos intensos. ...........................49

4.5.1 Relaciones de escala en la fuente. ……………………………………………...... 50

5 Enfoque semiempírico. Modelado Estadístico............................................................................53

5.1 Método O.S.A..............................................................................................................

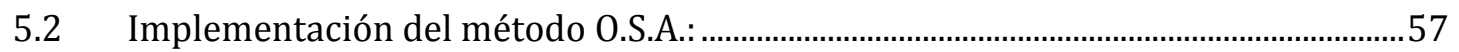

5.2.1 Datos del medio y de la función de Green empírica. ..............................................57

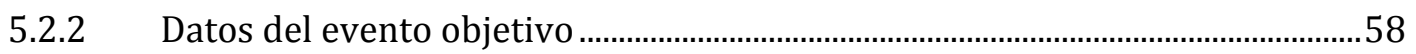

5.2.3 Obtención de la función temporal equivalente de la fuente. ..............................58

5.3 Simulaciones con el método O.S.A. ……………………………………………………....60

6 Enfoque semiempírico. Modelado Cinemático de la Ruptura ...................................................69

6.1 Método de modelado cinemático basado en los postulados de Irikura.....................71

6.2 Implementación del método de modelado cinemático..................................................75

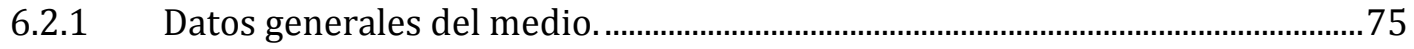

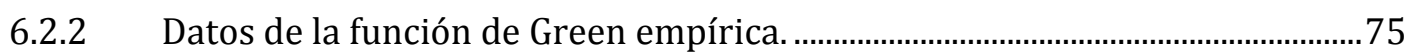

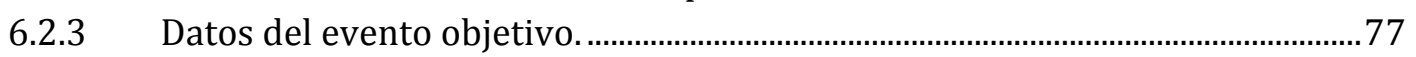

6.2.4 Función de Filtrado.........................................................................................

6.2.5 Obtención de la función temporal equivalente de la fuente. ..............................79

6.3 Simulaciones con el método de Irikura.............................................................................. 79

$7 \quad$ Aplicación al Cálculo estructural. .............................................................................................. 89

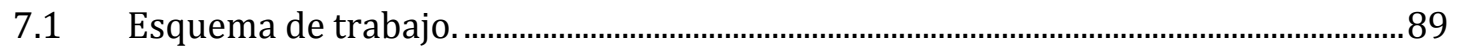

7.2 Simulación de terremotos en la zona de Granada.........................................................91

7.2.1 Contexto geológico y sismotectónico. ..................................................................... 91

7.2.2 Acción sísmica en un edificio de importancia normal ubicado en la zona universitaria de Cartuja (Granada) .................................................................................... 93

7.2.2.1 Espectros de respuesta elástica de la normativa.................................................93

7.2.2.2 Simulación por el modelo estadístico.............................................................95

7.2.2.3 Simulación por el modelo cinemático: .............................................................98

7.2.3 Acción sísmica en un edificio de importancia normal ubicado en la zona de la Facultad de Ciencias de la Universidad de Granada ......................................................... 101

7.2.3.1 Espectros de respuesta elástica de la normativa............................................. 101

7.2.3.2 Simulación por el modelo estadístico:_................................................................ 103

7.2.3.3 Simulación por el modelo cinemático. ……...................................................... 106

7.2.4 Acción sísmica en un edificio de importancia normal ubicado en Santa Fé

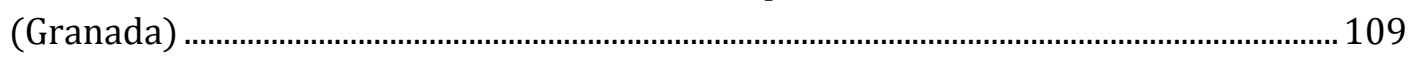

7.2.4.1 Espectros de respuesta elástica de la normativa:...........................................109

7.2.4.2 Simulación por el modelo estadístico:_..............................................................111

7.2.4.3 Simulación por el modelo cinemático. ..............................................................114

7.3 Discusión sobre la aplicación práctica de los modelos:...............................................117

8 Conclusiones y Futuras líneas de investigación. ....................................................................119 


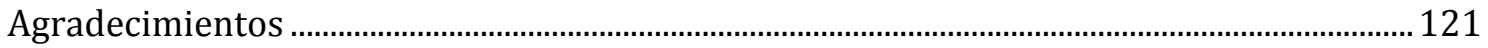

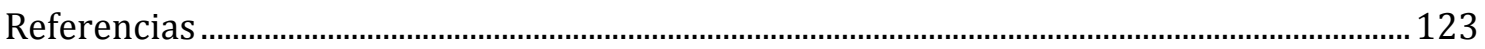

Anejo 1: Catalogo de terremotos utilizados para las simulaciones............................................127

Terremoto del 24 de Febrero de 1997, Agrón ............................................................................... 127

Terremoto del 5 de Enero de 1997, Sierra Elvira........................................................................... 129 


\section{INTRODUCCIÓN}

A lo largo de los estudios de ingeniería en la ETSICCP de Granada y sobre todo a en los primeros años donde me impartieron clase los profesores Gerardo Alguacil y Francisco Vidal, me ha llamado la atención la poca importancia relativa que se le daba al cálculo sísmico de estructuras, en estabilidad de taludes, etc. acudiendo siempre a unos parámetros simplificados de la normativa, para obtener una aceleración base y de ahí una acción sísmica de cálculo, totalmente estática.

Es decir, que con un parámetro estático solucionamos un problema dinámico, sin tener en cuenta lo que es en sí la acción de un terremoto que es eminentemente dinámica. Por eso, cuando se plateó la posibilidad de realizar este Trabajo fin de Máster sobre la acción sísmica, le vi rápidamente la utilidad. Esta tesina de Máster trata de encontrar una manera lo más sencilla posible de obtener una representación del sismo de proyecto en el dominio del tiempo para usarla en el cálculo estructural.

Lo primero que hay que pensar es qué posibilidades de representación de la acción sísmica tenemos, y cómo esta acción sísmica se recoge en la normativa. Luego hay que conocer y comprender qué aspectos de la sismología hay que tener en cuenta para representar un terremoto y qué trabajos hay publicados sobre el sobre la simulación y la predicción de movimientos sísmicos, para finalmente poder desarrollar herramientas que nos lleven a lograr una representación de la acción sísmica potencial en un determinado lugar.

Pues este camino descrito es el que nos llevará, en este documento, desde una contextualización de la acción sísmica en el cálculo estructural, a una explicación somera de los conocimientos, modelos y magnitudes de los sismos hasta llegar a los métodos de simulación de terremotos.

De los modelos de simulación el más útil es el enfoque semiempírico, que utiliza registros de eventos pequeños, a modo de función de Green empírica, para generar registros de terremotos mayores, con hipocentro en la misma zona que los eventos pequeños, y que se registra en la misma estación.

Se explicará con extensión el porqué de ser el más útil;, solo apuntar que los modelos con enfoque puramente teórico utilizan simplificaciones para conseguir calcular numéricamente los efectos de camino y de sitio. Con el enfoque semiempírico, estos efectos ya vienen introducidos con el uso de eventos pequeños, por lo que solo hay que desarrollar una función que recree la fuente de manera equivalente para la simulación. 
Como también se verá, los métodos que desarrollamos son dos: 1) los que generan una fuente equivalente de manera estadística, y 2) los que desarrollan la fuente equivalente a través de modelos de fuente cinemática.

El modelado estadístico que explicaremos es el de Ordaz, Singh y Arboleda (1995) que desarrollaron un esquema de suma estocástica a través de una función de distribución de probabilidad de los tiempos de retardo.

Y el modelado cinemático se basa en los postulados de Irikura, y su evolución a lo largo de estos años por otros autores, en los que se modeliza la función temporal equivalente de la fuente a través de variaciones consecuentes de la velocidad de ruptura en el plano de falla durante el evento sísmico.

Estos modelados se han programado en códigos del programa MATLAB, y de los cuales se pueden generar eventos objetivo a partir de registros de eventos pequeños, y de fijar las características del evento que queremos simular.

Con estos modelos, se generan los acelerogramas simulados, que podemos usar para el cálculo estructural, siguiendo un esquema de trabajo ordenado. Téngase en cuenta que la introducción de acelerogramas en programas de cálculo basados en el método de los elementos finitos, por ejemplo, no se pueden usar para el predimensionamiento de la estructura, pero si como comprobación de un modelo estructural ya fijado. Por ejemplo introduciendo la formación de rótulas plásticas, se puede ver cómo se comporta la estructura ante una acción sísmica modelizada con acelerogramas sintéticos. 


\section{OBJETIVOS}

El objetivo principal es el de generar acelerogramas sintéticos lo más realistas posibles ante la carencia de acelerogramas reales para la zona de estudio aplicables al cálculo dinámico directo de estructuras ante acciones sísmicas. Esto es de gran importancia en zonas sísmicas moderadas y bajas, debido a escasez de registros de fuerte movimiento de terremotos de magnitud media y alta.

En una zona de sismicidad moderada como la del sur-este de España, ocurre este problema. Tenemos actividad sísmica eventual, pero cuando ocurre genera graves daños, como se demuestra con solo repasar la sismicidad histórica.

Como solo se ha contado con una red de sismógrafos sólida en las últimas décadas, no se tienen registros de los terremotos históricos. Se sabe que los hay, pero no los hemos registrado instrumentalmente. De ahí la importancia que recae en este trabajo de investigación, el objetivo es difícil, simular los terremotos que pueden ocurrir en zonas de riesgo.

Para el logro del objetivo principal, plateemos unos objetivos parciales a desarrollar a lo largo de todo este documento.

> Contextualizar el objetivo principal en el ámbito del cálculo estructural, preguntándonos cuáles son las formas de representar la acción sísmica cuando se realiza el proyecto de una estructura.

$>$ Fundamentar el conocimiento en sismología, contestando preguntas como sobre los terremotos, como qué lo provoca, cómo se representa, cómo se mide y se compara, cómo se puede modelizar la fuente y los efectos de la propagación de las ondas, etc.

$>$ Estudiar los diferentes enfoques que se le puede dar a la simulación de terremotos.

Desarrollar modelos que sean capaces de generar registros de terremotos, de la manera más sencilla posible, para que sean una herramienta de utilidad.

Aplicación de estos modelados de terremotos al cálculo estructural, cómo conseguir "escalarlos" de manera orientativa a partir de la normativa vigente. 


\section{CONTEXTUALIZACIÓN EN EL CÁLCULO ESTRUCTURAL}

Aunque este documento está muy influenciado por los conceptos físicos de la sismología, no hay que perder de vista nuestro objetivo principal, que no es más, que el de encontrar una representación de la acción sísmica en el domino del tiempo para realizar el cálculo estructural.

No obstante, es claro que habrá que tener un conocimiento amplio de los procesos naturales que llevan a generar este tipo de acciones, pero también contextualizar todo dentro del campo del cálculo de estructuras y del marco normativo que lo rige.

Los terremotos son catástrofes naturales que no se pueden evitar, ni tampoco predecir, si pensamos en predicción como conocer que se van a producir en una zona, en un tiempo y con una magnitud determinados. Sin embargo, sí se conocen las zonas con más riesgo en las que se puede producir una sacudida sísmica de una determinada intensidad para un periodo de retorno dado, con lo cual se pueden mitigar los efectos destructores o dañinos con un adecuado diseño sismorresistente en las estructuras de esa zona.

Este diseño está regulado por normativas que tienen por objetivo proporcionar los criterios que se han de cumplir a la hora de considerar la acción sísmica en el proyecto. Y la dinámica de estructuras nos da las herramientas para realizar el diseño.

\subsection{La acción sísmica en el cálculo estructural.}

Es necesario medir de alguna manera el potencial destructivo de los terremotos para así poder conocer las acciones que se van a realizar sobre la estructura, y una vez conocidas, proyectar las estructuras para que, una vez construidas, sean capaces de resistir un escenario de esfuerzo máximo previsto a lo largo de su vida útil.

Como describe el profesor Amadeo Benavent en su libro "Estructuras Sismorresistentes", hay tres formas básicas de representar un terremoto a efectos de cálculo:

- mediante espectros elásticos de respuesta

- mediante espectros de input de energía

- mediante acelerogramas 


\subsubsection{Caracterización mediante espectros elásticos de respuesta.}

Los espectros elásticos de respuesta que se utilizan para caracterizar al terremoto de proyecto para una determinada región son la envolvente de los valores máximos de las diferentes respuestas ante un conjunto de sismos esperables en esa región con el periodo de retorno fijado.

El método de cálculo que usa estos espectros elásticos de respuesta se le conoce como el método del análisis modal espectral. Este método utiliza el desacoplamiento modal del método de superposición modal, pero, a diferencia de este, no proporciona una respuesta exacta para cada instante del tiempo, sino que obtiene la respuesta máxima de la estructura para cada modo de vibración y después realiza una combinación de estas mediante reglas aproximadas (la regla de la raíz cuadrada de la suma de los cuadrados, o la regla de la combinación cuadrática completa), con lo que se consigue una estimación del valor máximo total.

Los espectros elásticos de respuesta de proyecto proporcionan por tanto la pseudoaceleración absoluta máxima de respuesta que experimenta un sistema elástico de un grado de libertad sometido al terremoto de proyecto. Si se multiplica por la masa, se obtiene la fuerza de inercia máxima, que tiene que ser compensada por la estructura que se opone al movimiento. La estructura tiene que desarrollar una fuerza elástica opuesta a esta fuerza de inercia, por tanto, caracterizar un terremoto de proyecto por medio de espectros elásticos de respuesta, es caracterizarlo en términos de fuerza asumiendo que la estructura se mantendrá en régimen elástico y lineal.

Para tener completamente definido el terremoto de proyecto mediante el espectro elástico de respuesta es necesario conocer:

- la forma del espectro de proyecto normalizado, para el tipo de suelo sobre el que se pretende apoyar la estructura.

- el nivel del terremoto respecto al cual se ha normalizado el espectro y que normalmente viene expresado en términos de aceleración máxima del suelo.

- la fracción de amortiguamiento de la estructura.

Esta forma de caracterizar los terremotos de proyecto es la común en la normativa nacional y europea, por lo que se tratará más adelante cuando relacionemos la acción sísmica dentro del marco normativo.

\subsubsection{Caracterización mediante espectros de input de energía.}

El espectro de respuesta elástica, aunque es una importante medida con grandes aplicaciones, tiene limitaciones a la hora de cuantificar el daño potencial de un movimiento del terreno. Por ejemplo, además de otras limitaciones, al calcular la estructura caracterizando el sismo mediante el espectro elástico de respuesta no se incluyen los efectos de una respuesta inelástica estructural, la cual está generalmente relacionada con el daño. 
Esta deficiencia en la caracterización de la acción sísmica se puede solventar si vemos el terremoto como una cantidad de energía liberada en la fuente, transmitida por el terreno y que llega hasta la estructura, es decir, un input de energía en la estructura.

El input de energía sísmica para un sistema inelástico de un grado de libertad se puede cuantificar del siguiente modo:

$$
E=E_{H}+E_{K}+E_{S}+E_{\xi}
$$

Es decir, que es la suma de la energía histerética disipada, $E_{H}$, la energía cinética, $E_{K}$, la energía elástica (recuperable), $E_{S}$, y la energía disipada por el amortiguamiento, $E_{\xi}$.

El primer miembro de la ecuación, $E$, sería el efecto de la carga del terremoto expresada en términos de energía, y el segundo miembro, $E_{H}+E_{K}+E_{S}+E_{\xi}$, la capacidad sismoresistente de la estructura.

El input de energía total se suele expresar mediante la pseudo-velocidad equivalente, $V_{E}$, que se define como:

$$
V_{E}=\sqrt{\frac{2 \cdot E}{M}}
$$

donde M es la masa total del sistema.

Realizando los espectros de energía de entrada a partir de acelerogramas registrados se puede obtener una envolvente que sea el espectro de input de energía de proyecto para el cálculo sismorresistente de estructuras aplicando los métodos de energéticos basados en la teoría de Housner - Akiyama. Estos métodos se basan en la ecuación de balance energético expresada antes, y establecen la condición

$$
E \leq E_{H}+E_{K}+E_{S}+E_{\xi}
$$

para que la estructura sobreviva al terremoto de proyecto.

Como se verá cuando se explique el marco normativo, estos métodos energéticos aun no se contemplan como forma de caracterizar la acción sísmica en la estructura, por tanto son de gran importancia para la comprensión del comportamiento de la energía del sismo en las partes estructurales, pero de uso limitado por no estar incluido su cálculo en la normativa.

\subsubsection{Caracterización mediante acelerogramas.}

En los dos apartados anteriores se han descrito dos maneras de caracterizar la acción sísmica mediante espectros, es decir, en el domino de la frecuencia. Son espectros de proyecto en la normativa, por lo que son envolventes de espectros de registros que cubren la mayoría de los posibles eventos esperados en un lugar para un determinado periodo de 
ocurrencia. No se trata de una envolvente de todos los registros, por tanto, se trata de unas magnitudes orientativas para poder realizar el cálculo de la acción sísmica mediante métodos más o menos aproximados que sean válidos para la mayor parte de los casos.

La manera más objetiva de medir la acción sísmica dentro de la estructura es la de aplicar un cálculo dinámico directo a la misma, introduciéndole un acelerograma. El cálculo directo nos da la respuesta exacta del sistema sometido a las aceleraciones del suelo representadas por los acelerogramas a lo largo del tiempo que dura el evento. Además, si se consideran sistemas elastoplásticos, la única manera de conocer la respuesta dinámica exacta ante la acción sísmica es mediante el uso de estos cálculos dinámicos directos, pero para ello se requiere un importante esfuerzo de cálculo.

Por tanto, para proyectar adecuadamente una estructura mediante cálculos dinámicos directos nos surgen dos problemas a considerar. El primero, la cantidad y dificultad de cálculos a realizar y el segundo problema, es el de los acelerogramas a utilizar.

El tiempo de cálculo hace unos años era un gran impedimento que llevaba a solo usar este tipo de cálculos directos para estructuras singulares, pero hoy día con un conocimiento de métodos de cálculo, como el método de los elementos finitos, un conocimiento de la mecánica estructural y con un software adecuado, se pueden realizar este tipo de cálculos directos para cualquier tipo de estructura.

Respecto a los acelerogramas a utilizar, el problema se centra en la cantidad y en la calidad. Conseguir un conjunto de acelerogramas representativos de la peligrosidad sísmica de la zona donde se proyecta una determinada estructura para un periodo de tiempo determinado no es una labor fácil, y más aún si nos centramos en la problemática de nuestro entorno. Es aquí donde se palpa el objetivo de esta investigación: conseguir acelerogramas en la cantidad y calidad necesarias para realizar un cálculo estructural mediante el cálculo dinámico directo, de manera que se cumpla la normativa y se considere la peligrosidad sísmica lo más realista posible dentro de la zona donde se proyecta la estructura.

En un territorio como España y más concretamente en la parte sur-este que es una zona de sismicidad moderada, pero la zona española donde más peligrosidad existe, el problema para el uso de acelerogramas para el cálculo estructural es básicamente la inexistencia de registros que representen los terremotos destructivos que históricamente se conoce que han sucedido.

Pero volviendo a una perspectiva descriptiva, podemos considerar que los acelerogramas a usar pueden ser acelerogramas naturales, es decir, acelerogramas de eventos sísmicos ya ocurridos en la zona, y acelerogramas sintéticos, registros simulados de eventos plausibles en la zona. El uso de acelerogramas naturales se puede describir como enfoque empírico de simular posibles terremotos con registros pasados, en el cual, se espera que suceda lo que ya ha sucedido para realizar el cálculo estructural. Mientras que la simulación de una manera sintética, puede clasificarse en dos conjuntos. Un primer enfoque puramente teórico, en el que se utilizan los conocimientos teóricos de la fuente sísmica, los efectos del camino de las ondas y los efectos de sitio, para generar un registro sintético. 0 bien, usar 
registros de eventos más pequeños para generar eventos mayores a través de un escalado correcto. A esto se le conoce como enfoque semi-empírico, ya que utiliza tanto conocimientos empíricos como conocimientos teóricos. El mayor beneficio de este enfoque, es que solo hay que modelizar una fuente equivalente, ya que los efectos del camino y del sitio vienen implícitos en el registro del evento pequeño. Este enfoque es la columna vertebral de todo este trabajo, y se desarrollará con amplitud en los puntos siguientes.

La metodología para realizar el cálculo dinámico directo se podría secuenciar en los siguientes pasos:

1. Seleccionar los acelerogramas necesarios para representar la peligrosidad sísmica de la zona y cumplir la normativa.

2. Modelizar la estructura de manera lineal o no (elástica o elastoplástica) y calcular la respuesta de los acelerogramas seleccionados.

3. Se promedian las solicitaciones en la estructura para los acelerogramas, lo cual nos dará las solicitaciones de cálculo, y se compara con las resistencias asignadas a la estructura en el modelo para ver si la estructura cumple con las expectativas esperadas en el proyecto.

Para realizar este procedimiento, es necesario que previamente se haya realizado un dimensionamiento de la estructura, por tanto buscamos el comportamiento de la estructura ante los acelerogramas, se puede tomar como una puesta a prueba de lo previamente dimensionado.

\subsection{La acción sísmica en el marco normativo.}

La normativa sísmica de aplicación en España de manera obligatoria está constituida por:

- $\quad$ Norma de Construcción Sismorresistente: Parte general y edificación (NCSE-02)

- $\quad$ Norma de Construcción Sismorresistente: Puentes (NCSP-07)

Además de la normativa nacional de obligado cumplimiento en su ámbito de aplicación dentro del territorio nacional, existe la normativa europea del Eurocódigo 8, redactado por el Comité Europeo de Normalización (CEN) con la pretensión de unificar criterios y normativas nacionales. Como la normativa de obligado cumplimiento en el estado español son la NCSE-02 y la NCSP-07, nos centraremos en ellas para definir la acción sísmica tal y como la contemplan.

En la normativa, la acción sísmica vendrá dada o bien por acelerogramas, o bien por espectros de respuesta. De hecho, los sismos de proyecto se caracterizan normalmente por su espectro de respuesta elástica, siendo la aceleración máxima del terreno el valor de referencia para formar el espectro. 
El objetivo final de este documento es conseguir acelerogramas lo más realistas posibles para caracterizar el terremoto de proyecto, por lo que nos interesa conocer que restricciones impone la normativa de obligado cumplimiento para el uso de esta modalidad de caracterización.

El movimiento sísmico puede caracterizarse mediante conjuntos de acelerogramas horizontales y verticales compatibles con los espectros de respuesta de proyecto. En el apartado 3.7 Acelerogramas, del la NCSP-07, se puede leer:

"Los acelerogramas deberán ser elegidos entre los registrados, o generados artificialmente, de forma que se ajusten al espectro de respuesta elástica para un amortiguamiento del 5\%, [...]. Deberán, además, tener una duración representativa del movimiento esperable en cada caso.

Cuando el cálculo sísmico se realice utilizando acelerogramas, debido a su variabilidad, será necesario calcular la estructura con diferentes conjuntos de acelerogramas. En general, serán necesarios pares coherentes de acelerogramas horizontales y, cuando sea preciso considerar la componente vertical del sismo, tríos coherentes formados por dos acelerogramas horizontales y una vertical.

Los acelerogramas generados pertenecientes al mismo conjunto (par de dos acelerogramas horizontales o trío de dos horizontales y un vertical) deberán ser estadísticamente independientes."

La norma NCSE-02, en su apartado 3.6.1., viene a decir prácticamente lo mismo, salvo algunas matizaciones como que "Los cálculos deberán realizarse a partir de un número representativo de terremotos diferentes, que como mínimo serán 5, adoptando como solicitación de cálculo el promedio de los valores característicos obtenidos en cada uno".

Por tanto, para caracterizar el terremoto de proyecto de una determinada zona mediante acelerogramas, es necesario conocer el espectro de proyecto determinado por la normativa. Este espectro de proyecto se caracteriza por la clasificación del suelo y por la aceleración sísmica ce cálculo. A continuación determinaremos esa aceleración de cálculo a través de la información sísmica que nos dan las dos normativas, así como la caracterización del terreno, y de ello podremos obtener los espectros de respuesta elástica.

\subsubsection{Aceleración sísmica de cálculo.}

La aceleración sísmica de cálculo, $a_{c}$, se define como el producto:

donde

$$
a_{c}=S \cdot \rho \cdot a_{b}
$$

- $a_{b}$ es la aceleración sísmica básica, definida como el valor característico de la aceleración horizontal de la superficie del terreno en relación al valor de la aceleración de la gravedad. Este valor de $a_{b}$, y el del coeficiente de contribución, $K$, 
vienen detallados en el mapa de peligrosidad sísmica nacional (Fig. 3.1.) que se puede ver a continuación y en el Anejo 1 de la NCSE-02, donde se recoge un listado por municipios españoles con estos valores correspondientes a un periodo de retorno de 500 años.

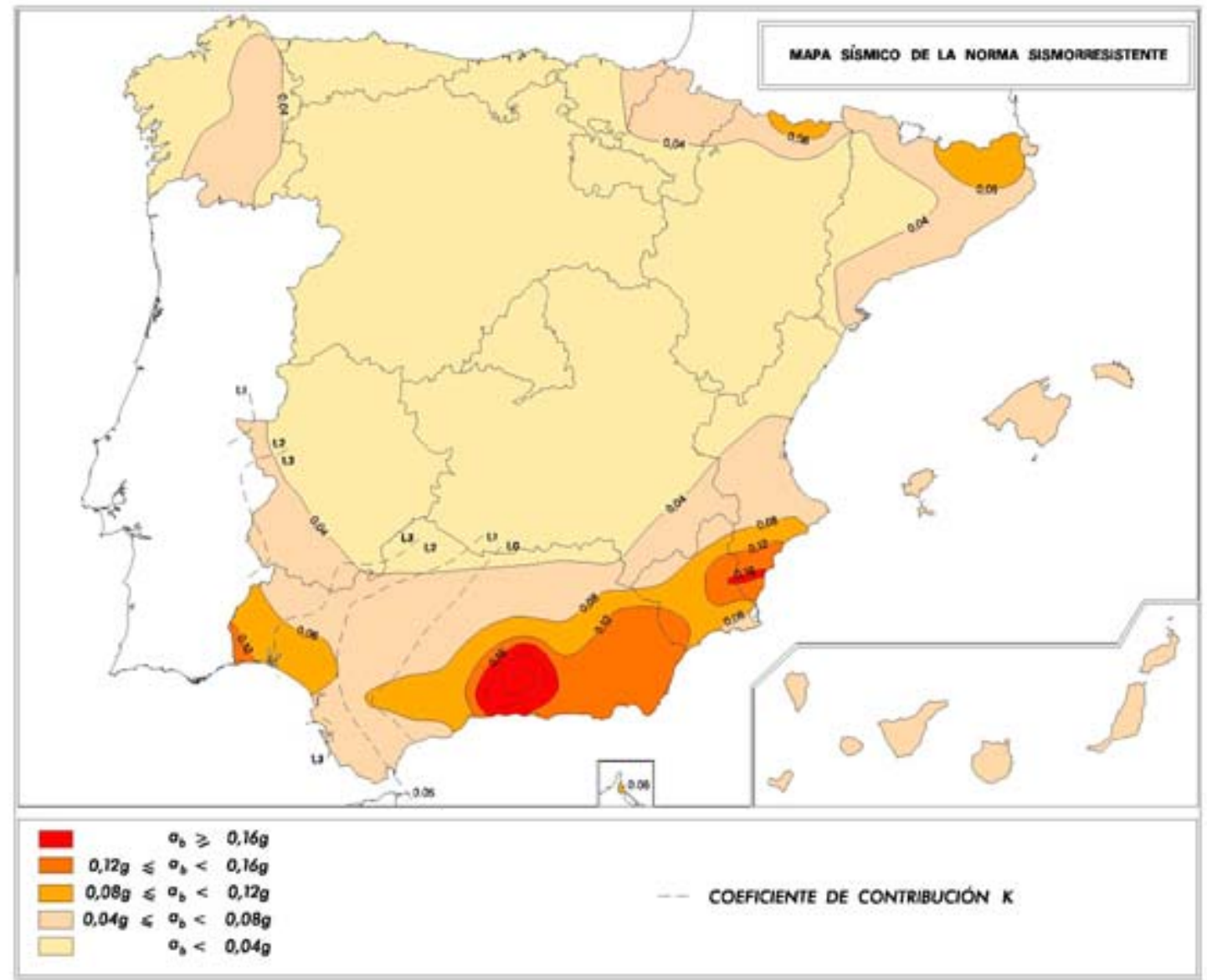

Figura 3.1. Mapa de peligrosidad sísmica. NCSE-02.

- $\quad \rho$ es un coeficiente adimensional de riesgo, función de la probabilidad aceptable d que se exceda $a_{c}$ en el periodo de vida para el que se proyecta la construcción. Toma los siguientes valores para la NCSE-02:

o para construcciones de importancia normal, $\rho=1.0$.

o para construcciones de importancia especial, $\rho=1.3$

Mientras para la NCSP-07, el valor viene determinado como producto de dos factores:

$$
\rho=\gamma_{I} \cdot \gamma_{I I}
$$

siendo $\gamma_{I}$, el factor de importancia, igual a 1 para importancia normal e igual a 1.3 para importancia especial, y $\gamma_{I I}$, un factor modificador para considerar un periodo de retorno diferente de 500 años. El producto representa la aceleración sísmica horizontal correspondiente a un periodo de retorno $P_{R}$. A falta de un estudio de peligrosidad específico de la zona de emplazamiento, se puede suponer que: 


$$
\gamma_{I I}=\left(P_{R} / 500\right)^{0.4}
$$

- $\quad S$ es el coeficiente de amplificación del terreno, que toma los siguiente valores:

0 para $\rho \cdot a_{b} \leq 0.1 g$,

$$
S=\frac{C}{1.25}
$$

o para $0.1 g \leq \rho \cdot a_{b} \leq 0.4 g$,

$$
S=\frac{C}{1.25}+3.33\left(\frac{\rho \cdot a_{b}}{g}-0.1\right) \cdot\left(1-\frac{C}{1.25}\right)
$$

o para $0.4 g \leq \rho \cdot a_{b}$

$$
S=1.0
$$

siendo $C$ el coeficiente del terreno que definiremos a continuación.

\subsubsection{Caracterización del terreno.}

Los movimientos del suelo provocados por un terremoto están influidos por el tipo de terreno. Por ello, es necesario llevar a cabo las investigaciones necesarias para identificar el tipo de terreno de acuerdo con las indicaciones recogidas. Como se puede ver en la Tabla 3.1., a cada tipo de terreno se le asigna un valor de coeficiente $C$. 
Tabla 3.1. Caracterización del terreno

\begin{tabular}{|c|c|c|}
\hline $\begin{array}{c}\text { Tipo de } \\
\text { terreno }\end{array}$ & Descripción & Coeficiente \\
I & $\begin{array}{c}\text { Roca compacta, suelo cementado o granular muy denso. } \\
\text { Velocidad de propagación de las ondas elásticas transversales } \\
\text { Vs }>750 \mathrm{~m} / \mathrm{s}\end{array}$ & 1,0 \\
\hline II & $\begin{array}{c}\text { Roca muy fracturada, suelos granulares densos o cohesivos duros. } \\
\text { Velocidad de propagación de las ondas elásticas transversales: } \\
750 \mathrm{~m} / \mathrm{s}>\text { Vs }>400 \mathrm{~m} / \mathrm{s}\end{array}$ & 1,3 \\
\hline III & $\begin{array}{c}\text { Suelo granular de compacidad media, o suelo cohesivo de } \\
\text { consistencia firme a muy firme. Velocidad de propagación de las } \\
\text { ondas elásticas transversales: } 400 \mathrm{~m} / \mathrm{s}>\text { Vs }>200 \mathrm{~m} / \mathrm{s}\end{array}$ & 1,6 \\
\hline IV & $\begin{array}{c}\text { Suelo granular suelto, o suelo cohesivo blando. Velocidad de } \\
\text { propagación de las ondas elásticas transversales o de cizalla } \\
\text { Vs }<200\end{array}$ & 2,0 \\
\hline
\end{tabular}

Este coeficiente $C$ depende de las características de los primeros 30 metros bajo la superficie, y de los espesores y rigideces de las capas de suelo superficial existentes en cada punto de la zona de emplazamiento, por lo que hay que tener en cuenta esta variación a la hora de usar un valor concreto de este parámetro.

\subsubsection{Espectros de respuesta elástica según la NCSE-02.}

Esta norma establece un espectro normalizado de respuesta elástica en la superficie libre del terreno, para aceleraciones horizontales, correspondiente a un oscilador lineal simple con un amortiguamiento de referencia del $5 \%$ respecto al crítico (Fig. 3.2), definido por los siguientes valores

$-\quad T<T_{A}$

$$
\alpha(T)=1+1.5 \cdot \frac{T}{T_{A}}
$$

- $\quad T_{A} \leq T \leq T_{B}$

$$
\alpha(T)=2.5
$$

- $\quad T<T_{B}$

$$
\alpha(T)=K \cdot \frac{C}{T}
$$

donde $\alpha(T)$ es el valor del espectro normalizado de respuesta elástica, $\alpha(T)=S_{a} / a_{c}$, $T$ es el periodo propio del oscilador en segundos, $K$ es el coeficiente de contribución, que tiene en cuenta la influencia de los distintos tipos de terremotos esperados en la peligrosidad 
sísmica de cada punto, $C$ es el coeficiente del terreno, y $T_{A}$ y $T_{B}$, los periodos característicos del espectro de respuesta, cuyos valores son:

$$
\begin{aligned}
T_{A} & =K \cdot \frac{C}{10} \\
T_{B} & =K \cdot \frac{C}{2.5}
\end{aligned}
$$

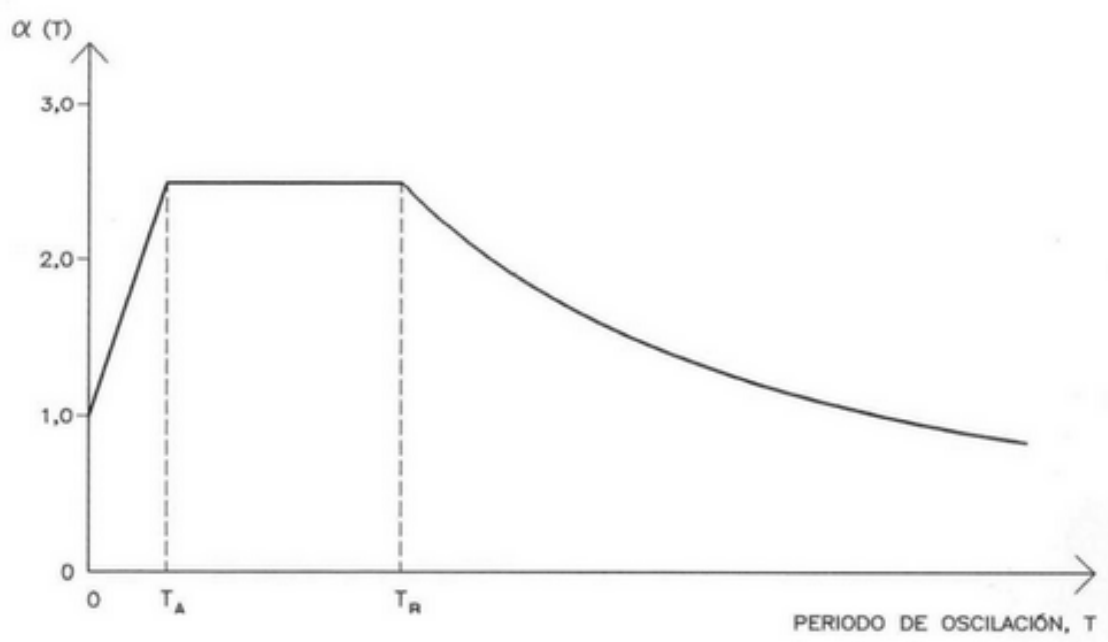

Figura 3.2. Espectro de elástico de respuesta característico de la normativa.

Para valores de amortiguamiento de la estructura diferentes del 5\% del crítico, los valores de $\alpha(T)$ para periodos $T \geq T_{A}$, se multiplicarán por el factor

$$
v=(5 / \Omega)^{0.4}
$$

donde $\Omega$ es el amortiguamiento de la estructura expresado como porcentaje del crítico. Para periodos $T<T_{A}$, las ordenadas espectrales se interpolarán linealmente entre los valores correspondientes a $T=0$ y $T=T_{A}$.

Cuando sea preciso considerar los movimientos verticales, se adoptará un espectro de respuesta elástica cuyas ordenadas espectrales sean el $70 \%$ de los valores correspondientes para movimientos horizontales.

\subsubsection{Espectros de respuesta elástica según la NCSP-07.}

Para las componentes horizontales de la acción sísmica, se considerará el siguiente espectro de respuesta elástica de aceleraciones $S_{a}(T)$, correspondiente a un oscilador lineal simple.

$$
-\quad 0 \leq T<T_{A}
$$

$$
S_{a}(T)=\left(1+\frac{T}{T_{A}}(2.5 v-1)\right) \cdot a_{c}
$$


- $\quad T_{A} \leq T \leq T_{B}$

$$
S_{a}(T)=2.5 v \cdot a_{c}
$$

$-T_{B} \leq T \leq T_{C}$

$$
S_{a}(T)=2.5 v \cdot \frac{T_{B}}{T} \cdot a_{c}
$$

$-\quad T<T_{C}$

$$
S_{a}(T)=2.5 v \cdot \frac{T_{B} \cdot T_{C}}{T} \cdot a_{c}
$$

donde $a_{c}$ es el valor de la aceleración de cálculo, $v$ el factor corrector del amortiguamiento que se obtiene de la expresión

$$
v=(5 / \zeta)^{0.4} \geq 0.55
$$

válida para $\zeta>1 \%$, siendo $\zeta$ el índice de amortiguamiento, en porcentaje, cuyos valores aparecen en el apartado 4.2.3.3. de esta norma para cada tipo de sismo y de estructura.

$T_{A}, T_{B}$ y $T_{C}$ son los valores de los periodos que delimitan el espectro, dependen del tipo de sismo de cálculo y del tipo de terreno de cimentación del puente.

Se define el sismo último de cálculo como el sismo con un periodo de retorno de 500 años, que resulta de multiplicar la acción del sismo básico por un factor $\gamma_{I}$, el factor de importancia, que es igual a 1 para importancia normal e igual a 1.3 para importancia especial. Se denomina sismo frecuente de cálculo, al sismo con un periodo de retorno de 100 años que resulta de multiplicar la acción del sismo frecuente por el factor $\gamma_{I}$. Se denomina sismo de construcción, al sismo con un periodo de retorno no menor a cinco veces la duración de la etapa constructiva.

Dadas estas definiciones previas, tenemos que para el sismo último de cálculo:

$$
\begin{gathered}
T_{A}=K \cdot \frac{C}{10} \\
T_{B}=K \cdot \frac{C}{2.5} \\
T_{C}=K \cdot(2+C)
\end{gathered}
$$

y para el sismo frecuente de cálculo y para el sismo de construcción:

$$
\begin{gathered}
T_{A}=K \cdot \frac{C}{20} \\
T_{B}=K \cdot \frac{C}{5} \\
T_{C}=K \cdot(1+0.5 \cdot C)
\end{gathered}
$$


con $K$ y $C$ el coeficiente de contribución y el coeficiente de terreno respectivamente.

A falta de estudios más detallados, el espectro correspondiente a la componente vertical podrá obtenerse simplificadamente a partir del horizontal multiplicando por un factor igual a 0.7 . 


\section{SIMULACIÓN DE TERREMOTOS. FUNDAMENTOS}

Como se ha expresado en el apartado anterior de contextualización, la utilidad del uso de registros sintéticos lo más realistas posibles para el cálculo estructural por medio del cálculo directo para una zona donde existe peligrosidad sísmica pero sin registros, es clara.

En esta sección nos centraremos en los fundamentos necesarios para conocer los enfoques teórico y semi-empírico de la simulación de terremotos. Incidir en el enfoque teórico, que se escapa del objetivo marcado, es para tener una idea clara de las ventajas que nos arrojan los métodos semi-empíricos, basados en el uso de registros de eventos menores a modo de funciones de Green empíricas, y así poder tener la ventaja de que los efectos del camino y del sitio vayan implícitos dentro del registro del evento menor. Pero antes de entrar en los distintos enfoques teórico y semi-empírico, es necesario ahondar en la base matemática y sismológica en los cuales se desarrollan.

\subsection{Fundamentos matemáticos.}

A continuación se realiza un resumen de conceptos relativos a los fundamentos matemáticos de series temporales que se van a usar a lo largo de los siguientes apartados.

\subsubsection{Transformada de Fourier.}

Se define la Transformada de Fourier como:

$$
F(u(t))=u(\omega)=\int_{-\infty}^{+\infty} u(t) e^{i \omega t} d t
$$

Y también se define la Transformada Inversa de Fourier como:

$$
F^{-1}(u(\omega))=u(t)=\frac{1}{2 \pi} \int_{-\infty}^{+\infty} u(\omega) e^{-i \omega t} d \omega
$$

La función $u(t)$ se dice que está en el dominio del tiempo, mientras que su correspondiente función $u(\omega)$ está en el dominio de la frecuencia. A partir de estas definiciones, se pueden deducir estas relaciones: 
- Regla de escala:

$$
\begin{gathered}
F(u(t / a))=|a| u(a \omega) \\
u(t / a)=|a| F^{-1}(u(a \omega))
\end{gathered}
$$

- Regla de diferenciación:

$$
u(t)^{\prime}=-i \omega u(\omega)
$$

- Teorema del cambio:

$$
F(u(t+a))=u(\omega) e^{-i \omega a}
$$

\subsubsection{Convolución.}

Consideradas dos series temporales $f(t)$ y $g(t)$., se define la convolución de estas funciones como

$$
f(t) * g(t)=\int_{-\infty}^{+\infty} f(\tau) \cdot g(t-\tau) d \tau
$$

A continuación (Fig. 4.1) se puede ver una explicación visual de la convolución:

o Se expresa cada función en términos de una variable $\tau$.

o Se refleja una de las funciones:

$$
g(\tau) \rightarrow g(-\tau)
$$

o Se añade un tiempo de desplazamiento t, lo que permite que $g(t-\tau)$ se deslice a lo largo del eje $\tau$.

o Se hace $t$ igual a $-\infty$ y se va deslizando hasta llegar a $+\infty$. Siempre que las dos funciones se corten, se hace la integral de su producto. En otras palabras, calcular el promedio ponderado desplazado de la función $f(\tau)$, donde la función peso es $g(-\tau)$. La forma de onda resultante es la convolución de las funciones $f(t)$ y $g(t)$.

La convolución con pulsos simples por lo general se traduce en una suavización de la serie temporal original. Por ejemplo, la convolución con una función rectangular produce el mismo resultado que un promediado entre datos adyacentes. Si $f(t)$ es un impulso unitario (en el siguiente punto se definirá como delta de Dirac), el resultado de este proceso es simplemente $g(t)$, que se denomina por tanto la respuesta del impulso. 
Propiedades de la convolución son:

- Conmutatividad:

$$
f * g=g * f
$$

- Asociatividad:

$$
\begin{gathered}
f *(g * h)=(f * g) * h \\
a(f * g)=a f * g=f * a g
\end{gathered}
$$

con $a$ un escalar

- Distributividad:

$$
f *(g+h)=(f * g)+(f * h)
$$

- Derivación:

$$
(f * g)^{\prime}=f^{\prime} * g=f * g^{\prime}
$$

- Teorema de convolución (transformadas de Fourier y Lapalce):

$$
F(f * g)=F(f) \cdot F(g)
$$
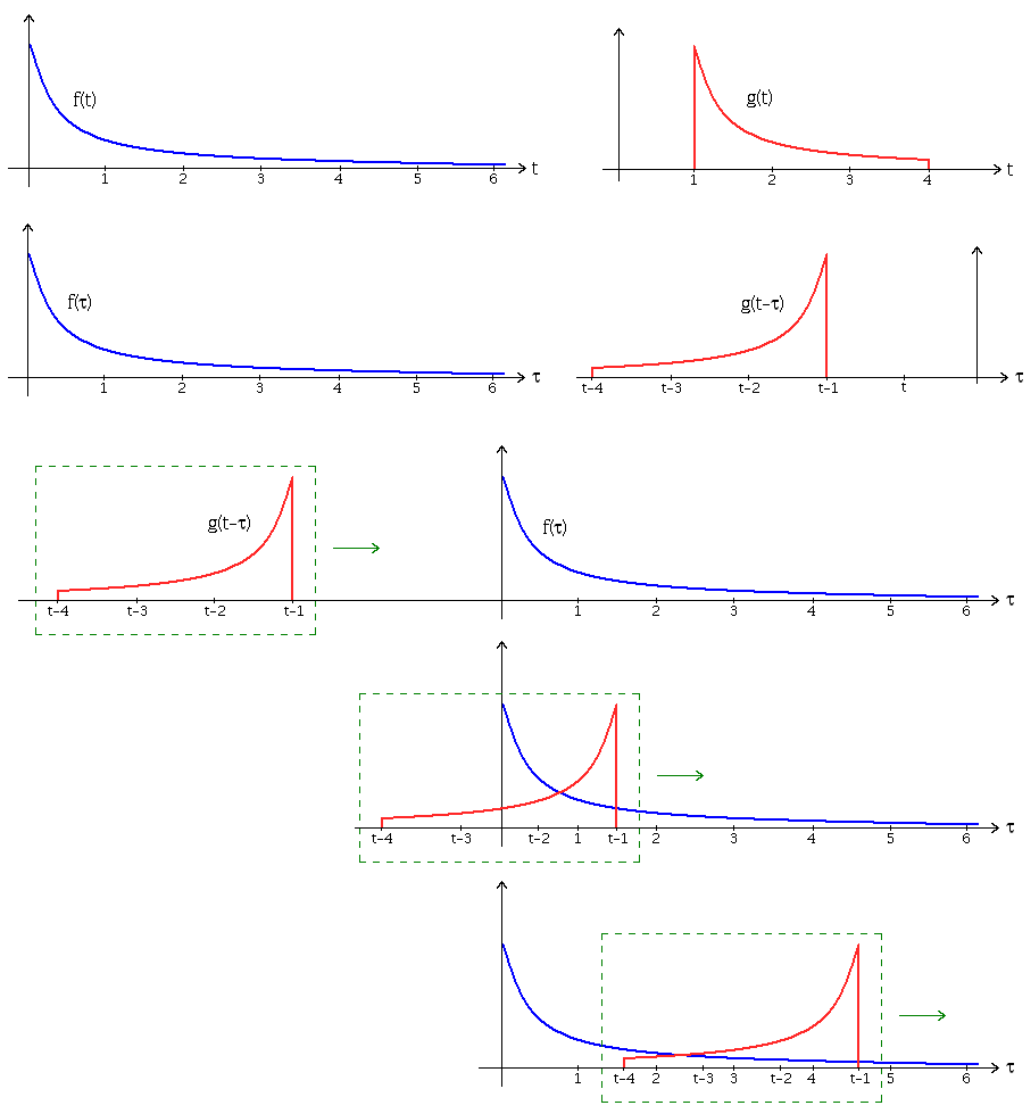

Figura 4.1. Representación gráfica del proceso de convolución. 


\subsubsection{Función delta de Dirac.}

La función delta de Dirac, $\delta(t)$, se define como:

$$
\begin{gathered}
\delta(t)=0 \text { para } t \neq 0 \\
\delta(t)=\infty \text { para } t=0 \\
\int_{-\infty}^{+\infty} \delta(t) d t=1
\end{gathered}
$$

Esta es la expresión de la delta de Dirac para una variable $t$ continua. Pero para nuestro uso, con los cálculos numéricos, es muy complicado trabajar con variables continuas y si muy habitual el uso de variables discretas, en las cuales se conocen las variables cada una determinado frecuencia de paso. Por tanto es mejor conocer la delta de Dirac para una variable $t$ discreta.

Partiendo de la propiedad anterior en la que la integral de función en todo $\mathcal{R}$ vale la unidad, e imaginándonos que se trata de un impulso infinito en torno al 0 , es fácil ver que esa función en un espacio discreto se podría ver como un impulso de valor la unidad en el 0 . A esto se le conoce como función impulso unitario, y su expresión para una variable $t$ discreta seria:

$$
\begin{gathered}
\delta(t)=0 \text { para } t \neq 0 \\
\delta(t)=1 \text { para } t=0 \\
\sum_{-\infty}^{+\infty} \delta(t)=1
\end{gathered}
$$

Para tener un impulso unitario en un tiempo determinado $t_{o}$, en vez de un impulso unitario en el cero, solo basta introducir una nueva variable $t-t_{o}$, con lo que conseguimos introducir un retardo a la función impulso unitario de valor $t_{o}$.

La transformada de Fourier de la delta de Dirac vendrá dada por:

$$
F\left(\delta\left(t-t_{o}\right)\right)=\int_{-\infty}^{+\infty} \delta\left(t-t_{o}\right) e^{i \omega t} d t=e^{-i \omega t_{o}}
$$

con lo que si se tratara de la delta de Dirac centrada en cero, $\delta(t)$, su valor seria la unidad. Por tanto, la convolución de una serie temporal con la función delta nos devuelve la función original sin ningún cambio.

$$
u(t) * \delta(t)=u(t)
$$


Si se quiere aplicar un retardo a una serie dada, por las propiedades mostradas, la función delta puede ser utilizada para provocar un desplazamiento temporal de la serie original un tiempo $t_{o}$.

$$
u(t) * \delta\left(t-t_{o}\right)=u\left(t-t_{o}\right)
$$

\subsection{Fundamentos sismológicos.}

En esta sección nos centraremos en resumir la base científica relativa al conocimiento de los terremotos, que nos hará falta para comprender los métodos de simulación de acelerogramas. ${ }^{1}$ Nos centramos en conceptos sismológicos referentes a la fuente de las ondas sísmicas y de como la energía radiada está relacionada con las propiedades físicas de la fuente. Todo esto es de una gran amplitud, por lo que solo cogeremos los aspectos que más falta hacen para poder comprender y desarrollar los métodos de simulación que más adelante se enunciarán.

\subsubsection{Fallas, Tensor momento y Mecanismo de los terremotos.}

Antes de profundizar en cómo se producen unos desplazamientos a una distancia determinada del epicentro de un terremoto, es más lógico pensar en que los genera, cómo representar esa generación y cómo poder comparar los eventos sísmicos para poder decir que uno es mayor que otro. Para ello en este epígrafe se describen las fallas como generadoras de terremotos, y se explican los conceptos de tensor momento, de momento sísmico y de magnitud momento para poder medir y comparar terremotos en función de su "tamaño".

\subsubsection{Fallas sísmicas.}

Los movimientos intensos del terreno, como lo son las sacudidas sísmicas, pueden ser debidos a explosiones, actividad volcánica, a derrumbamientos en cavidades subterráneas, y, los más importantes, a la actividad tectónica en una región, que produce rupturas en las discontinuidades llamadas fallas. Son estas últimas las que solo vamos a considerar como fuentes sísmicas en el ámbito de este trabajo. Por tanto solo vamos a considerar los terremotos como movimientos provocados por la ruptura a través de un plano de falla con una orientación arbitraria (Fig. 4.2). Por lo que hay que poder describir y orientar este mecanismo de manera numérica para poder usar estos datos para describir el evento sísmico.

\footnotetext{
${ }^{1}$ Para este apartado nos basamos en tres libros divulgativos de suma importancia en esta parte de la ciencia. "Introduction to Seismology" de Peter M. Shearer, "Quantitative Seismology" de Aki y Richards, y "Seismic Ray Theory" de Vlastislav Cervený. Para ahondar más en los conceptos que se exponen, se recomienda acudir a estos textos donde se explican con mucha mayor amplitud.
} 
El plano de falla se define por medio de los siguientes parámetros:

- Acimut, $\phi$, orientación de la línea de falla respecto del norte (strike).

- Buzamiento, $\delta$, ángulo respecto el plano horizontal (dip).

La dirección y la magnitud de la ruptura se representan por medio del vector deslizamiento contenido en el plano de falla (Fig. 4.2). El vector deslizamiento (cuya modulo se conoce como $D$ o $\Delta u$ ) (slip), también conocido como salto de falla, se define como el movimiento del bloque de arriba (techo) respecto del bloque de abajo (muro).

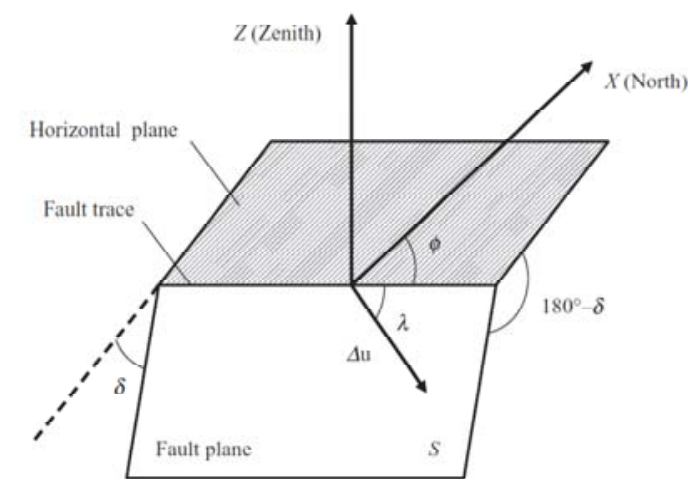

Figura 4.2. Parámetros que describen la orientación, buzamiento y dirección de deslizamiento en una falla

El ángulo del vector deslizamiento puede medirse en el plano vertical, salto vertical, que será el movimiento en altura entre los dos bloques (plunge), y en el plano de falla, que se denota como $\lambda$ (rake), que es al ángulo formado por la línea de falla y el vector deslizamiento.

Un movimiento hacia arriba del techo, es decir, un movimiento caracterizado por la compresión entre los bloques, es el que tiene una falla inversa. Siendo la falla normal, la de un estado tensional de tracción, donde el techo se mueve hacia abajo (Fig. 4.3). Por tanto, la forma más básica de definir el mecanismo de ruptura de una falla es por medio del acimut, $\phi$, buzamiento, $\delta$, el modulo del vector deslizamiento, $D$, y su ángulo respecto del plano horizontal $\lambda$. 


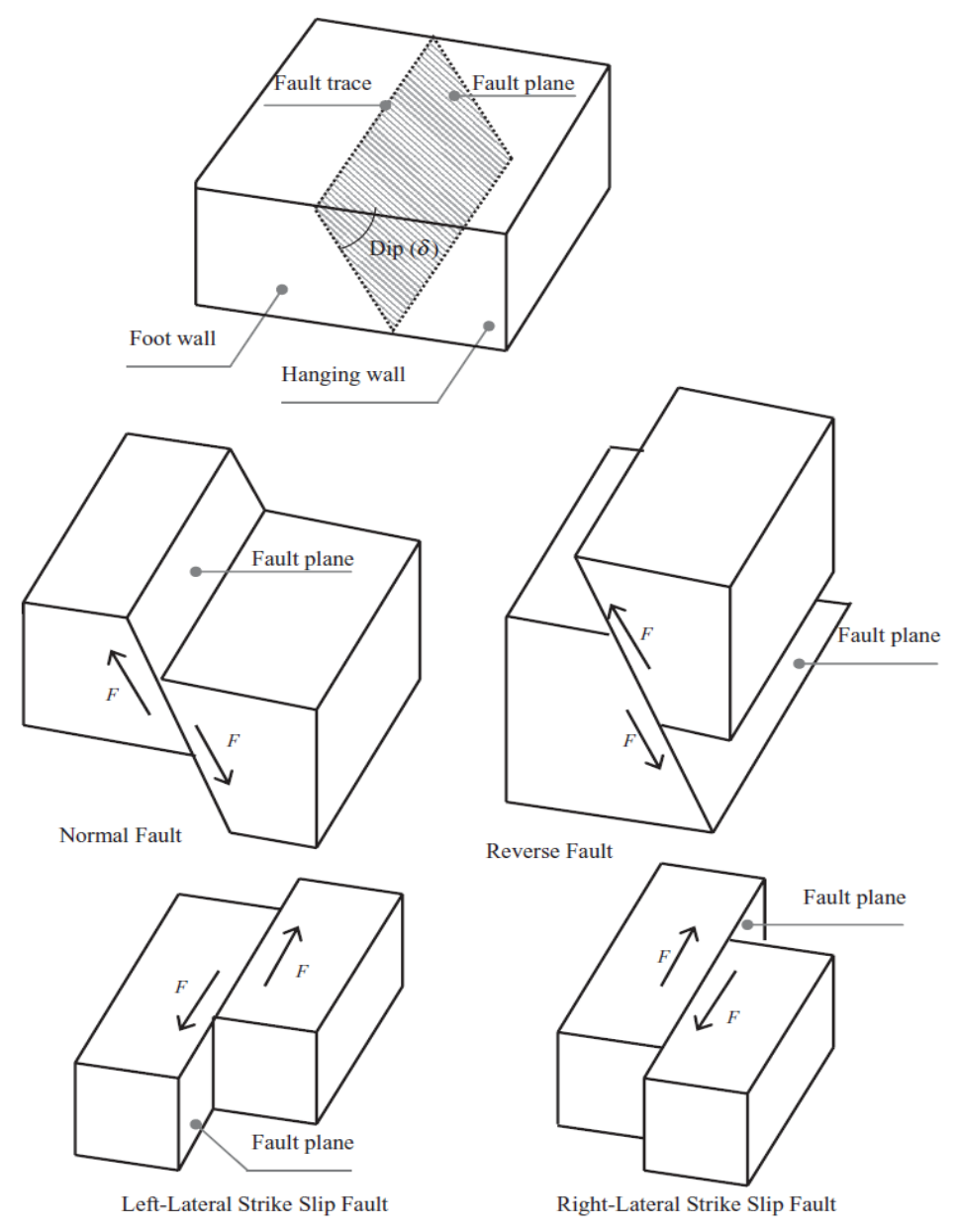

Figura 4.3. Clasificación de las fallas según el movimiento

Otros parámetros que hay que tener en cuenta en la definición de la fuente sísmica son la profundidad focal, el hipocentro, el epicentro, la distancia epicentral (epicentro-estación) e hipocentral (entre foco y el sitio de observación), (Fig. 4.4).

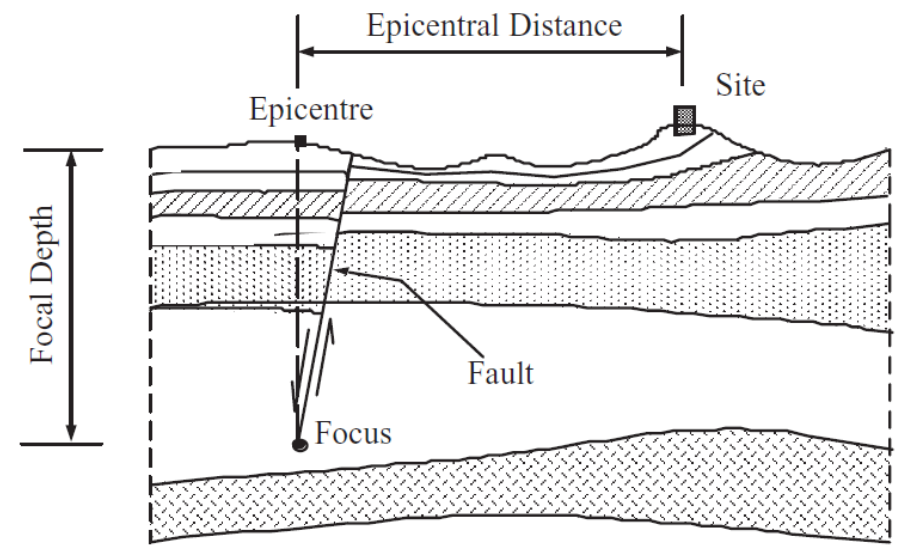

Figura 4.4. Distancias entre epicentro, hipocentro y estación 


\subsubsection{Tensor momento y Momento sísmico escalar.}

Un terremoto se suele modelar como un deslizamiento a través de una superficie de discontinuidad en el interior de un medio elástico. Este deslizamiento tiene que venir provocado por un estado de tensiones que se liberan cuando se produce la ruptura, es decir, el terremoto. Echando mano de la mecánica vectorial, y considerando que la fuente de la ruptura es puntual, la distribución de fuerzas puede ser equiparada a otro sistema de fuerzas equivalente que produce exactamente el mismo campo de desplazamientos para el modelo de falla.

Las fuerzas internas resultantes de una explosión o de la liberación de tensión en una falla deben actuar en direcciones opuestas con el fin de que haya un equilibrio de fuerzas y de momentos. Podríamos tener dos vectores de fuerza de magnitud $\mathrm{F}$, apuntando en direcciones opuestas, separados por una distancia d, a esto se lo denomina un par de fuerzas. Alternativamente, los vectores pueden estar separados en una dirección perpendicular a la orientación fuerza, en este caso el equilibrio de momentos no se conserva, a menos que también existe un par complementario que lo equilibre (Fig. 4.5).
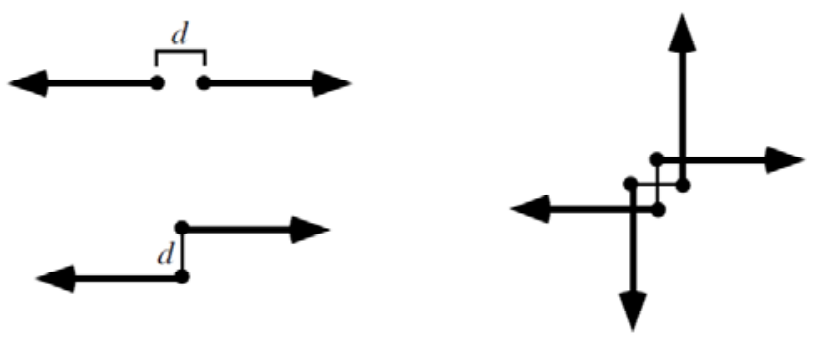

Figura 4.5. Pares de fuerzas separados una distancia determinada. Izqda) Modelo de par simple. Dcha) Modelo de par doble.

Se define el momento $M_{i j}, i, j=1,2,3$, en un sistema de coordenadas cartesianas como el par de fuerzas apoyadas en una dirección $i$ y separadas en la dirección $j$. El valor de $M_{i j}$ viene dado por el producto $f \cdot d$, y si $d$ tiende a cero en la fuente puntual, tenemos el tensor de momentos en ese punto.

$$
M_{i j}=\left(\begin{array}{lll}
M_{11} & M_{12} & M_{13} \\
M_{21} & M_{22} & M_{23} \\
M_{31} & M_{32} & M_{33}
\end{array}\right)
$$

Donde el significado de cada una de las componentes puede verse en la siguiente imagen (Fig.4.6.) 

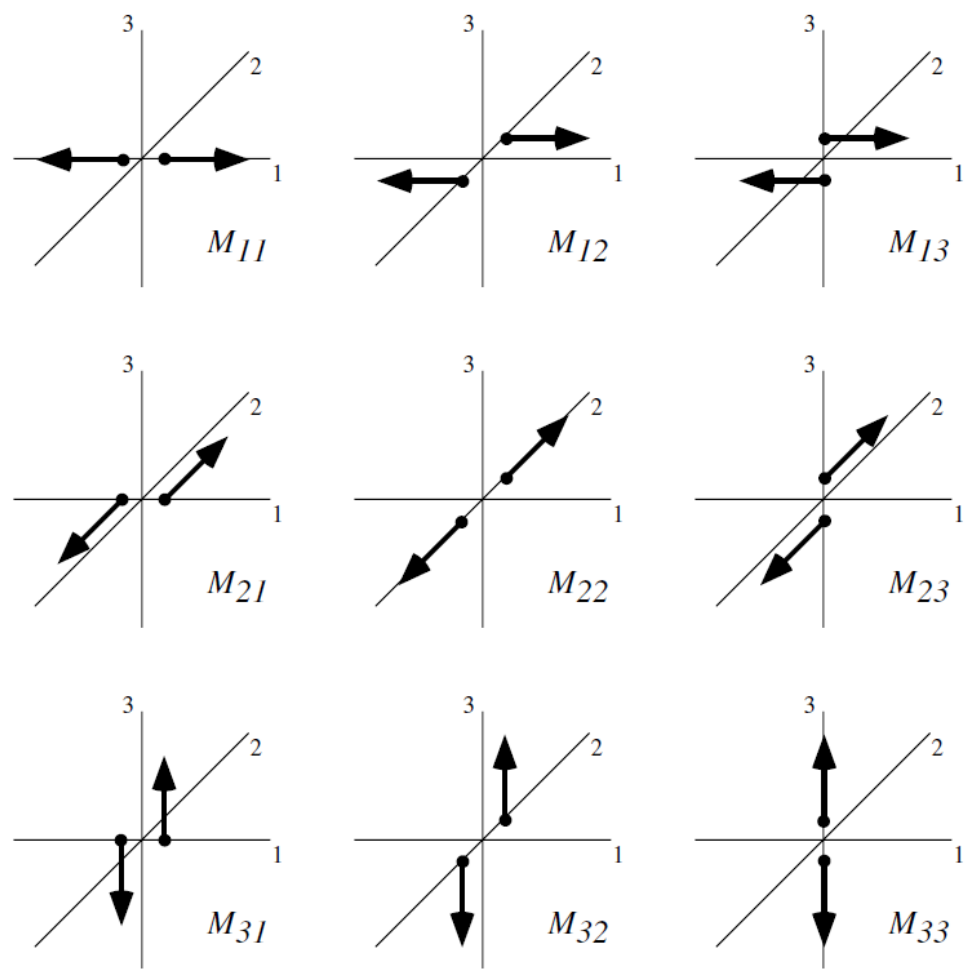

Figura 4.6. Componentes del tensor momento

La condición de equilibrio de los momentos hace que este tensor sea simétrico, por tanto, $M_{i j}=M_{j i}$, con lo que tenemos solo seis elementos independientes. El tensor momento sísmico nos da una representación de las fuerzas generadas que actúan en el punto de ruptura del medio elástico. Aunque se trata de una idealización, se trata de una buena aproximación para la respuesta sísmica de fuentes a la distancia suficiente como para poder asimilarlas a rupturas puntuales. Incluso para rupturas más grandes, pueden ser representadas de esta manera como suma de rupturas puntuales en diferentes posiciones.

La representación con el tensor de momentos del mecanismo que genera un terremoto puede ser sustituido por una representación equivalente a través de una magnitud escalar y unas direcciones a modo de descripción geométrica de la falla.

Como ya se ha dicho, se puede demostrar que la energía sísmica radiada por la fuente puede ser modelada por medio de un par de fuerzas que provocarían un campo de desplazamientos equivalente al que se produce. Con lo que se puede definir el momento sísmico escalar como

$$
M_{o}=\mu \cdot D \cdot A
$$

Donde $\mu$ es el modulo de cizalla, $D$ es el desplazamiento de la falla, y $A$ es el área de ruptura en la falla. Este $M_{0}$ sería el módulo del vector momento equivalente al par de fuerzas mencionado anteriormente. 


\subsubsection{Mecanismos focales.}

Y la descripción geométrica se puede realizar por medio de lo que se conoce como mecanismo focal, que es la representación estereográfica (Fig. 4.7) de los ángulos que definen el plano de falla, es decir, del azimut (strike), $\phi$, buzamiento (dip), $\delta$, y su ángulo respecto del plano horizontal (rake) $\lambda$.
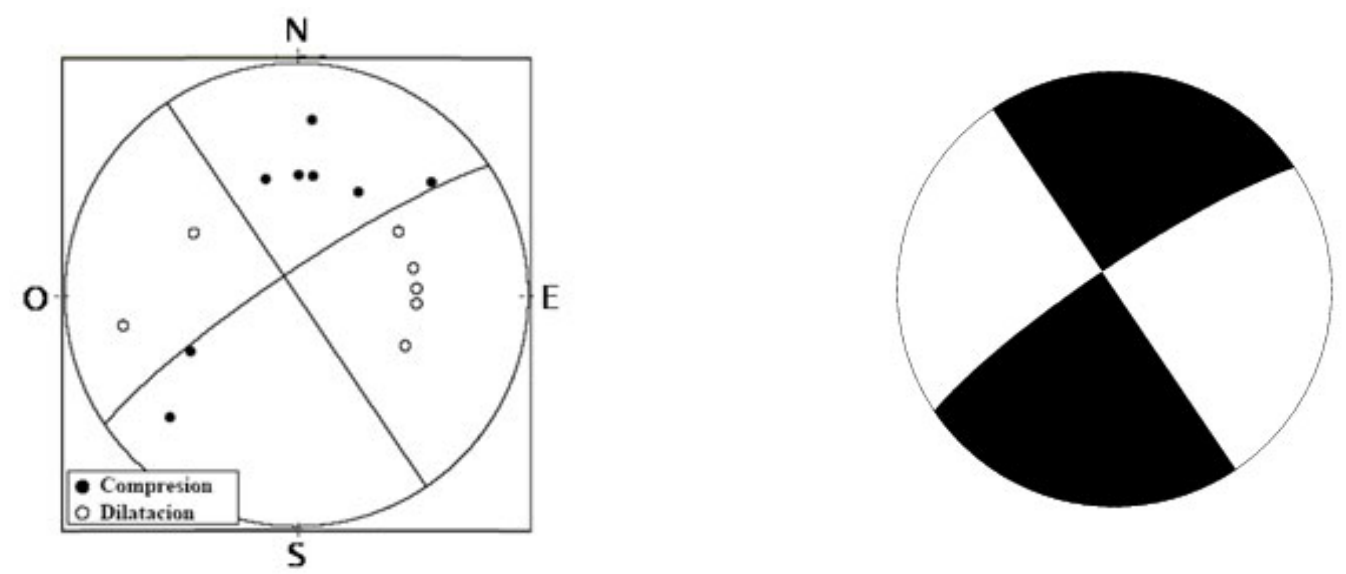

Figura 4.7. Representación estereográfica del mecanismo focal (izq.), y la representación simplificada más usual (dcha.)

Podemos poner algunos ejemplos de fallas tipo con sus mecanismos focales equivalentes (Figs. 4.8, 4.9., 4.10 y 4.11). Con estas idealizaciones podemos representar el mecanismo de la falla que genera los terremotos de una manera más o menos sencilla.

- Falla normal: Dip $=45^{\circ}$ Rake $=-90^{\circ}$
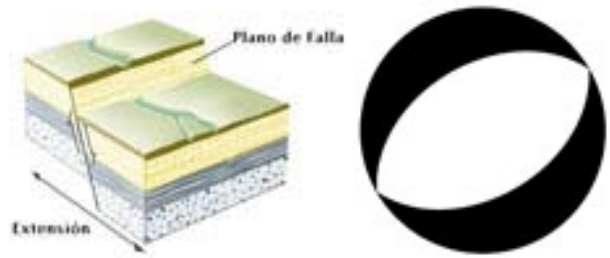

Figura 4.8. Falla normal y mecanismo focal asociado

- Falla inversa (reverse): Dip $=45^{\circ}$ Rake $=90^{\circ}$
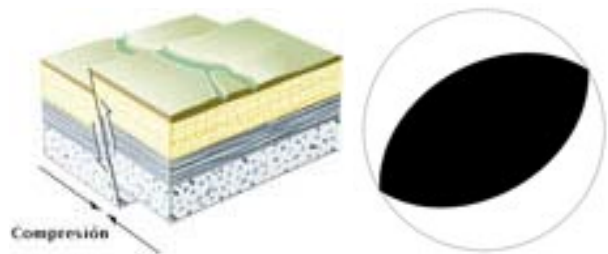

Figura 4.9. Falla inversa y mecanismo focal asociado

- Falla transcurrente (strike slip): puede ser lateral derecha (Dip $=90^{\circ}$ Rake $=$ $180^{\circ}$ ) o lateral izquierda $\left(\right.$ Dip $=90^{\circ}$ Rake $=0^{\circ}$ ) 


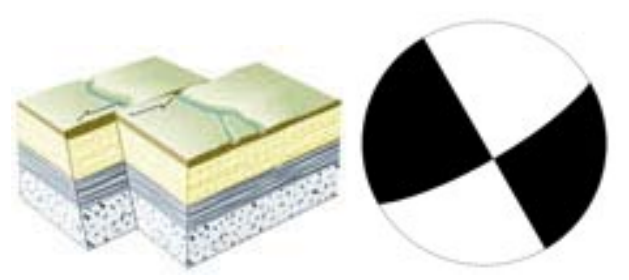

Figura 4.10. Falla transcurrente y mecanismo focal asociado

- Falla oblicua: combinación de normal o inversa con transcurrente

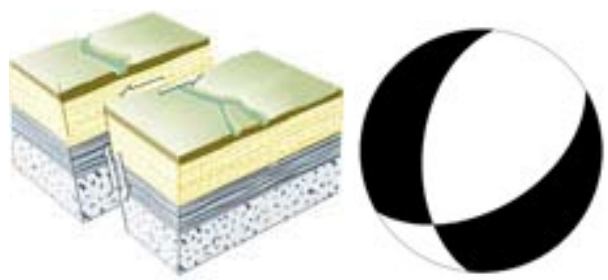

Figura 4.11. Falla oblicua y mecanismo focal asociado

\subsubsection{Magnitud de un terremoto.}

Por razones históricas, la medida más conocida de tamaño de un terremoto es la magnitud del sismo. En la actualidad hay diferentes escalas de magnitud, pero todas están basadas en la mayor amplitud que se registra en un sismograma. Este es una de los parámetros más fáciles de medir y es la razón de la popularidad de las escalas de magnitud.

En 1930, Charles Richter introdujo lo que ahora se conoce como magnitud local, $M_{L}$, que se determina a través de la mayor amplitud $A$ del registro con el sismógrafo Wood-Anderson. De los estudios de Richter se han desarrollado expresiones empíricas como la de Bullen y Bolt (1985):

$$
M_{L}=\log _{10} A+2.56 \log _{10} \Delta-1.67
$$

donde $A$ es la amplitud máxima del desplazamiento del suelo en micrones $\left(10^{-6} \mathrm{~m}\right)$ y $\Delta$ es la distancia en kilómetros. Esta fórmula es válida para $10<\Delta<600 \mathrm{~km}$.

Esta escala es un método rápido para determinar el tamaño de los diferentes eventos, pero estaba pensada para la zona de California, por lo que hubo que desarrollar magnitudes más generales que englobasen el resto de zonas, como la magnitud $m_{b}$ conocida como "body wave magnitude", y la magnitud $M_{S}$, "surface wave magnitude", magnitudes de ondas internas y superficiales, respectivamente. Estas escalas se calibraron de acuerdo con la magnitud $M_{L}$ para pequeños eventos locales en California, pero tenían problemas de saturación para eventos mayores de $\sim 6.9$.

Dados estos problemas, Kanamori (1977) desarrolló la magnitud momento, con lo que se conseguía una escala de magnitud relacionada con el momento sísmico escalar, definido en el apartado anterior, a través de la expresión: 


$$
M_{w}=\frac{2}{3} \log _{10} M_{o}-10.7
$$

donde como se ha dicho $M_{o}$ es el momento sísmico, expresado en dina-cm. ${ }^{2}$ La ventaja de esta escala es que está relacionada directamente con una propiedad física del mecanismo que genera los eventos sísmicos, y no se satura para grandes terremotos, como si ocurría con el resto de escalas.

\subsection{Teorema de representación. La función de Green.}

El teorema de representación fue desarrollado por Maruyama (1963) y Burridge y Knopoff (1964), que describen que el desplazamiento del terreno causado por un deslizamiento a lo largo de una falla, se puede expresar como la convolución de una función de deslizamiento en la falla (efecto de la fuente), con una función de Green, la cual representa la respuesta del terreno, es decir, los efectos del camino y del sitio. Una expresión somera podría ser la siguiente

$$
u(t)=s(t) * G(t)
$$

donde $s(t)$ es la función fuente, $G(t)$ es la función de Green elástica que contiene la fuente y al receptor. Si lo pasásemos todo al dominio de la frecuencia, tendríamos una expresión aun más sencilla, donde la convolución pasaría a producto, y solo se trataría del producto de funciones. Pero ahondemos algo más en la formulación, expresando el teorema de representación de manera completa.

Si el deslizamiento en la falla es una función dependiente del tiempo y de la posición en la falla, el desplazamiento sísmico según el eje $i, u_{i}(\vec{x}, t)$ está relacionada con la historia de desplazamientos espacio-tiempo, $\Delta u_{i}(\vec{\xi}, \tau)$, en el plano de falla $\Sigma(\vec{\xi})$, y la función de Green $\mathrm{G}_{i j}(\vec{x}, t ; \vec{\xi}, \tau)$, como se expresa en la conocida ecuación desarrollada por Aki y Richards (1980),

$$
u_{i}(\vec{x}, t)=\int_{-\infty}^{+\infty} d \tau \int_{\Sigma}\left[\Delta u_{i}(\vec{\xi}, \tau)\right] \cdot c_{j k p q} \cdot \frac{\partial \mathrm{G}_{i p}(\vec{x}, t ; \vec{\xi}, \tau)}{\partial \xi_{q}} \cdot v_{k} \cdot d \Sigma(\vec{\xi})
$$

con $i, j, k, p, q=1,2,3$. La expresión está en notación indicial, donde los índices repetidos indican la suma sobre ese índice. $v$ es el vector normal unitario al plano de falla $\Sigma$, y $c_{j k p q}$ son las constantes elásticas definidas por

$$
c_{j k p q}=\lambda \delta_{j k} \delta_{p q}+\mu\left(\delta_{j p} \delta_{k q}+\delta_{j q} \delta_{k p}\right)
$$

\footnotetext{
${ }^{2}\left(10^{5}\right.$ dina $=1 \mathrm{~N} ; 10^{7}$ dina-cm $\left.=1 \mathrm{~N}-\mathrm{m}\right)$.
} 
donde $\lambda$ y $\mu$ son las constantes de Lamé, que se relacionan con el módulo de Young, $E$, y el coeficiente de Poisson, $v, y \delta_{j k}$ es la delta de Kronecker.

$$
\mu=\frac{E}{2(1+v)}=G
$$

donde $G$ es aquí el módulo de rigidez, y

$$
\lambda=\frac{E \cdot v}{(1+v)(1-2 v)}
$$

Por ejemplo, para un medio isótropo, homogéneo e infinito, $c_{j k p q}$ viene dado por

$$
\begin{gathered}
c_{1111}=c_{2222}=c_{3333}=\lambda+2 \mu \\
c_{1122}=c_{2233}=c_{3311}=\lambda \\
c_{1212}=c_{1221}=c_{2323}=c_{2332}=c_{3131}=c_{3113}=\mu
\end{gathered}
$$

La expresión del teorema de representación implica que el desplazamiento de cualquier punto en el medio está determinada por la convolución espacio temporal de la distribución de deslizamientos con la función de Green integrada sobre la superficie de la falla.

En un medio isótropo, homogéneo e infinito, esta función de Green puede ser expresada:

$$
\begin{gathered}
\mathrm{G}_{i p}(\vec{x}, t ; \vec{\xi}, \tau)=\frac{1}{4 \pi \rho}\left(3 \gamma_{i} \gamma_{p}-\delta_{i p}\right) r^{-3} \int_{\frac{r}{\alpha}}^{\frac{r}{\beta}} t^{\prime} \delta\left(t-\tau-t^{\prime}\right) d t^{\prime}+ \\
+\frac{1}{4 \pi \rho \alpha^{2}} \gamma_{i} \gamma_{p} r^{-1} \delta\left(t-\tau-\frac{r}{\alpha}\right)+\frac{1}{4 \pi \rho \beta^{2}}\left(\gamma_{i} \gamma_{p}-\delta_{i p}\right) r^{-1} \delta\left(t-\tau-\frac{r}{\beta}\right)
\end{gathered}
$$

donde $r$ es la distancia hipocentral, es decir, $|\vec{x}-\vec{\xi}|, \alpha$ es el vector unitario desde el punto del plano de falla $\vec{\xi}$ al punto del receptor $\vec{x}, \alpha$ la velocidad de las ondas P y $\beta$ la velocidad de las ondas S.

El primer término de la función de Green se comporta como $r^{-2}$ y el segundo y tercero como $r^{-1}$, convirtiéndose en dominantes sobre $r^{-2}$ cuando $r \rightarrow \infty$. El primer término es el término denominado de campo cercano, mientras que los otros dos son los términos de campo lejano.

En general, los movimientos intensos del terreno que se producen en el campo cercano son los que más interesan para el cálculo estructural por su capacidad de generar daños, aunque en campo lejano también se pueden producir grandes daños para eventos de mucha importancia (magnitudes altas). 
Si tomamos una fuente puntual que genera un impulso, el término de campo lejano también tendrá una forma impulsiva proporcional a la magnitud del impulso fuente, atenuado como $r^{-1}$, y su espectro es proporcional a $\omega^{0}$. El término de campo cercano tendría una forma impulsiva dentada con un incremento de la amplitud en los tiempos comprendidos entre los límites $\frac{r}{\alpha}$ y $\frac{r}{\beta}$. El área encerrada en esta forma impulsiva tiene una dependencia con la distancia proporcional a $r^{-1}$ y un espectro proporcional a $\omega^{-1}$ en el rango de periodos menores a

$$
T_{s-p}=\frac{r}{\beta}-\frac{r}{\alpha}
$$

Los términos de campo lejano por lo tanto se imponen en el rango de periodos cortos incluso en las zonas cercanas a la fuente. Para periodos mayores a $T_{s-p}$, el término de campo cercano es efectivamente un impulso tan bueno como en campo lejano. Se puede concluir que los términos de campo cercano y campo lejano tienen una igual importancia dependiendo de la distancia y del rango de periodos con el que se esté trabajando.

\subsubsection{Modelado de la fuente.}

Como ya se ha dicho anteriormente, en el teorema de representación, el movimiento intenso del terreno provocado por un evento sísmico se representa por la distribución de deslizamientos en la rotura de la falla, o efectos de la fuente, y la función de Green. En esta sección trataremos de todo lo relativo a la fuente.

Hay dos maneras de caracterizar el movimiento de la falla en la fuente de un terremoto, el modelo cinemático y el modelo dinámico. En el modelo cinemático se basa en funciones de desplazamientos espacio - temporales sobre el plano de falla, sin tener en consideración los factores físicos concernientes a las condiciones de esfuerzos actuantes en el proceso. El modelo dinámico tiene en cuenta el proceso físico de la fractura en la zona de la fuente.

A pesar de no tener en cuenta el proceso tensional que da lugar al evento sísmico, con el modelo cinemático se obtienen importantes resultados a la hora de interpretar los movimientos intensos del terreno y en la estimación de la distribución de rupturas y deslizamientos en el plano de falla.

El sencillo modelo cinemático de Haskell (1964), describe la fuente a partir de la longitud $(L)$ y anchura $(W)$ de la falla, del desplazamiento final $(D)$, de un parámetro tiempo conocido como "rise time" $\left(\tau_{r}\right)$ (o tiempo de subida) y de la velocidad de ruptura $\left(V_{r}\right)$. A continuación describiremos con mayor profundidad este modelado de la fuente.

\subsubsection{Modelo de Haskell. Parámetros.}

Los desplazamientos que ocurren a ambos lados de una falla durante un terremoto son permanentes, no se retorna al estado inicial tras el seísmo. Así, la representación de las 
fuerzas másicas equivalentes del campo de desplazamientos debería incluir un cambio permanente en las fuerzas aplicadas.

Además, el desplazamiento no es instantáneo, pero ocurre con una duración finita de la ruptura. Se pueden incluir estas propiedades generalizando la representación del tensor momento para ser dependiente del tiempo.
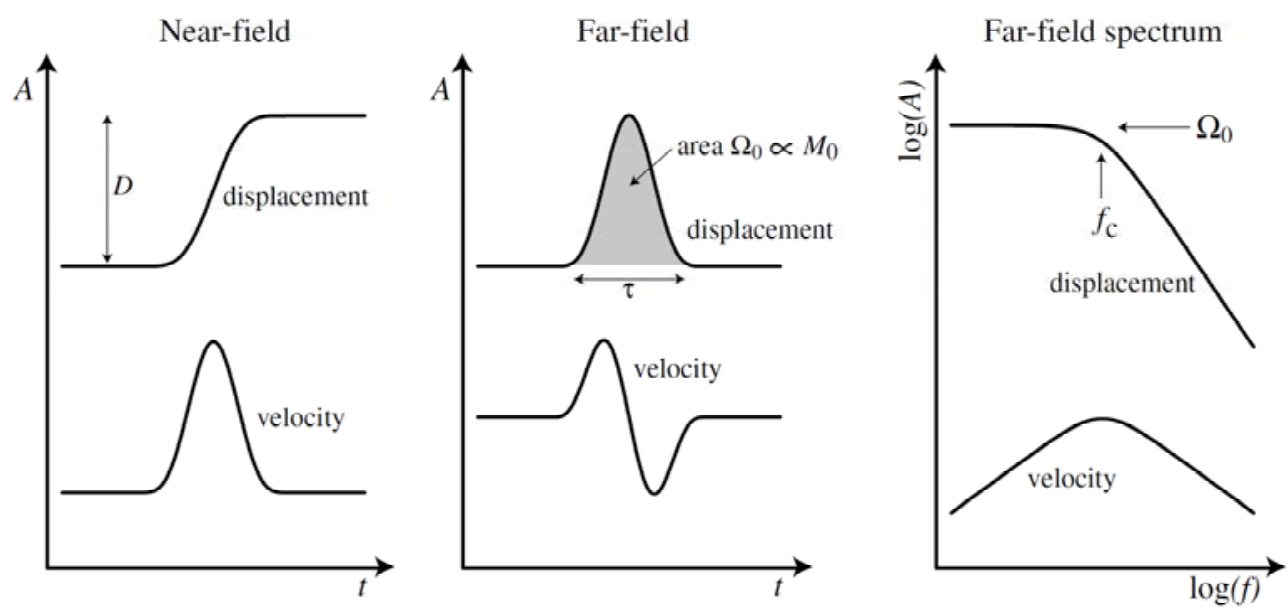

Figura 4.12. Relación entre los desplazamientos y velocidades de campo cercano y lejano

Cojamos una componente del tensor momento, expresándola como $M(t)$, con una forma similar a la curva de los desplazamientos en campo cercano (near-field) mostrada en la imagen anterior. $M(t)$ también será proporcional a la respuesta dinámica del campo lejano (far-field), tal como se puede observar en la llegada de ondas P o S. Se trata de un pulso de desplazamiento y por tanto no hay una deformación permanente después de que las ondas pasen.

La mayoría de los sismómetros miden la velocidad, $\dot{u}(t)$, o la aceleración, $\ddot{u}(t)$, en vez de medir los desplazamientos. Por tanto, lo que realmente se está grabando tiene una o dos derivadas temporales. En el domino de la frecuencia, el efecto de la derivada temporal es el de multiplicar el espectro por $\omega$, lo cual no deja de ser una ventaja, y además, estos registros de velocidades se han mejorado en el rango de las altas frecuencias respecto de los registros de desplazamientos.

En el estudio de estructura de la Tierra, por lo general, poco importa si se utiliza la velocidad antes que el desplazamiento, se asume una derivación extra para la fuente cuando estamos modelando las formas de onda. Sin embargo, cuando se estudian las fuentes sísmicas, de la velocidad se obtiene el desplazamiento. Esto se realiza mediante la integración del registro de velocidad y normalmente implica una corrección de la respuesta del instrumento. El objetivo es obtener un registro imparcial de $\dot{M}(t)$ en la fuente por lo que vamos a suponer para el resto de esta sección que estamos midiendo el desplazamiento en el campo lejano. 
Si generalizamos el concepto de momento escalar definido anteriormente, $M_{o}=\mu D A$, para que sea dependiente del tiempo, se puede definir

$$
\dot{M}(t)=\mu \frac{\partial}{\partial t}[A(t) D(t)]
$$

donde $M_{o}$ será el área bajo la función $\dot{M}(t)$, y por tanto el cambio en la función $M(t)$ la podremos expresar como

$$
M_{o}=\mu \bar{D} A
$$

donde $\mu$ es el modulo de cizalla, $\bar{D}$ es el desplazamiento promediado a lo largo de la falla, y $A$ es el área de la falla.
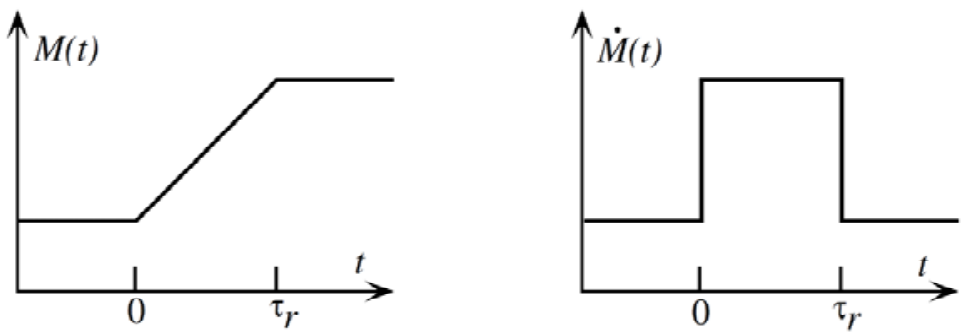

Figura 4.13. Idealización del modelo de fuente de Haskell

Consideremos la función de ruptura en la fuente (idealizada como puntual) como una función rampa, como en la figura anterior. Su correspondiente función derivada, $\dot{M}(t)$ y el desplazamiento del pulso en campo lejano será una función rectangular o también conocida como pulso unitario. A esta idealización de la ruptura se la conoce como modelo de fuente de Haskell (Fig. 4.13).

En terremotos para los cuales se puede aproximar la ruptura como una fuente puntual, el modelo de fuente de Haskell ofrece una muy buena descripción de la respuesta en campo lejano, y como idealización de la ruptura, se puede usar para comprender el comportamiento en campo cercano.

A la duración del pulso, $\tau_{r}$, se lo conoce como "rise time", es decir, el tiempo necesario para que el deslizamiento en un punto particular de la falla alcance su valor final.

El modelo de Haskell se podría definir como un modelo de falla con propagación unidimensional desde el punto de vista del campo lejano. Supone que el plano de falla es rectangular de dimensiones $L x W$, donde $L$ es la longitud de la fractura y $W$ la anchura. La ruptura empieza y termina en el plano de falla a lo largo de $L$ con una velocidad de ruptura $V_{r}$. 
La estimación de la longitud $L$ es sencilla ya que se puede obtener mediante el estudio de las ondas largas. No obstante, existe una expresión que la relaciona con el momento sísmico $M_{o}$ (Yamanaka y Shimazaki, 1990)

$$
\log _{10} L=\frac{1}{2}\left(\log _{10} M_{o}-23.58\right)
$$

donde $L$ se expresa en $\mathrm{km}$, y $M_{o}$ en dina-cm.

Numerosos estudios han comprobado que una buena estimación de la anchura es considerarla como la mitad de la longitud de la falla, por tanto

$$
W=\frac{L}{2}
$$

El desplazamiento final es difícil de conocerlo directamente, pero una estimación proporcional a él es el momento sísmico, que como se ha visto antes, es el producto del modulo de cizalla, del área de la falla $(L x W)$ y del deslizamiento final.

$$
D=\frac{M_{o}}{\mu A}
$$

Estudios sobre la velocidad de ruptura concluyen que se puede estimar en un rango de entre el 80 y el $90 \%$ de la velocidad de las ondas S.

Y para concluir con la estimación de los parámetros asociados con este modelo simplista de Haskell, Savage (1972) asumió que el rise time es igual al tiempo de recorrido del frente de ruptura sobre la mitad de la anchura de la falla, con lo que tenemos que

$$
\tau_{r}=\frac{W}{2 V_{r}}=\frac{L}{4 V_{r}}
$$

Con estos parámetros se puede definir el comportamiento de una falla desde el punto de vista del campo lejano. Para el campo cercano la cosa se complica bastante más. El modelo de propagación unidireccional de Haskell es una sobre-simplificación del proceso de ruptura. Para un modelo más realista, sería deseable que en un determinado punto se inicie la ruptura de manera unidireccional y luego se vaya propagando a lo largo de las dos dimensiones del plano de falla. Esto se puede conseguir con modelos de nucleación como en Savage (1966) que toma una velocidad de ruptura uniforme para toda la ruptura.

Un importante principio en sismología es la superposición lineal, la en la cual la respuesta ante una gran ruptura de falla puede ser representada como la suma de las respuestas de un número limitado de rupturas de falla más pequeñas. Así, se pueden modelar rupturas como la integración de fuentes puntuales de desplazamientos en la superficie de la falla.

Como se explicará en el apartado correspondiente a las simulaciones basadas en el enfoque teórico, este modelo simplista de Haskell no puede simular de manera 
satisfactoria los movimientos ricos en altas frecuencias dado que la fuente sísmica es mucho más compleja. Por ello se mejora el modelo con enfoques como la aleatoriedad en los parámetros de la fuente, o teniendo en cuenta que la función de deslizamiento y la propagación de la ruptura varían irregularmente en el plano de falla en un sentido determinista.

\subsubsection{Efectos de directividad.}

Para terremotos en los que la ruptura se extiende más en el tiempo y en geometría, se debe generalizar e incluir los efectos de directividad. Examinemos a modo de ejemplo cómo sería el caso de una ruptura a lo largo de una falla siguiendo una dirección como muestra la imagen (Fig. 4.14).

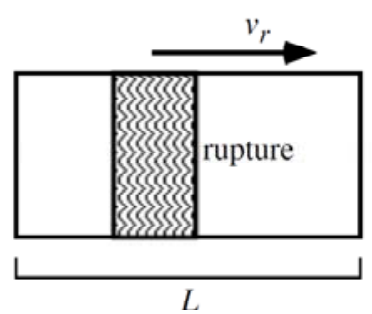

Figura 4.14. Propagación de la ruptura de manera lineal

Se trata de la propagación de la ruptura a lo largo de una longitud $L$ de la falla de izquierda a derecha, con una velocidad de ruptura de $V_{r}$. En el campo lejano se observaría una ruptura desde cada punto de la falla con una diferencia de tiempo (Fig. 4.15), por ejemplo, en el caso en el que la ruptura se dirigiera hacia el observador, la duración aparente de la ruptura, $\tau_{d}$, para las ondas P sería:

$$
\tau_{d}=L\left(\frac{1}{V_{r}}-\frac{1}{\alpha}\right)
$$

Mientras que el tiempo observado para una ruptura que se aleja de del observador, tendríamos que:

$$
\tau_{d}=L\left(\frac{1}{V_{r}}+\frac{1}{\alpha}\right)
$$




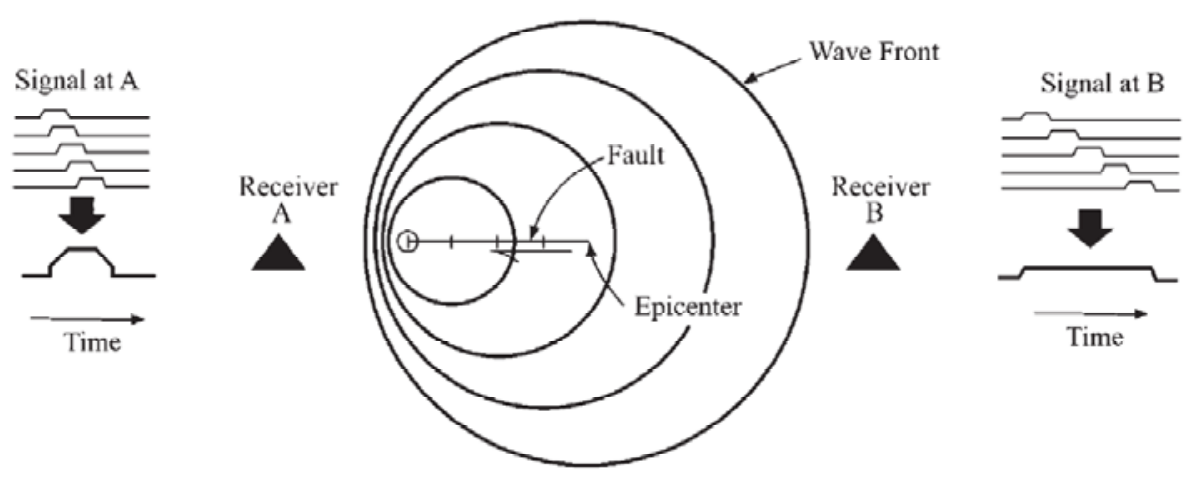

Figura 4.15. Síntesis del efecto de directividad

En general, $\tau_{d}$, es función de la orientación de la falla respecto del observador, y de la dirección y velocidad de la ruptura. A los cambios en $\tau_{d}$ como función de la localización del observador (o receptor) es lo que se conoce como efectos de directividad.

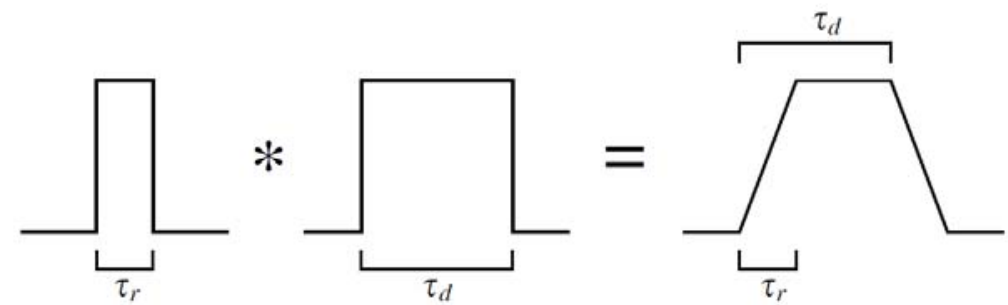

Figura 4.16. Modelo de falla de Haskell visto como convolución de dos funciones rectangulares con anchuras dadas por el rise time y el tiempo de duración de la ruptura

Imagínese ahora una ruptura real en la que el desplazamiento se puede describir como una función rampa. La forma del impulso de desplazamiento en campo lejano se dará por la convolución de dos funciones rectangulares, una de anchura $\tau_{r}$, rise time, y el otro de ancho $\tau_{d}$, el tiempo de duración aparente de la ruptura (Fig. 4.16). El pulso resultante será una función trapecio. Esto se denomina el modelo de falla Haskell y es válido para un modelo simple de una fuente lineal. Se puede demostrar que el área del trapecio es proporcional al momento escalar, $M_{o}$, durante todo el evento. La anchura del trapezoide variará con un azimut de la falla, así como con la variación del tiempo aparente de ruptura $\tau_{d}$

Como $M_{o}$ es proporcional al área bajo la función trapecio, y se debe de conservar para todo el evento, los impulsos más cortos tendrán amplitudes más grandes, y para impulsos más largos, amplitudes más pequeñas. Por lo tanto debemos esperar ver mayores amplitudes y duraciones más cortas de los pulsos en la dirección de propagación de la ruptura y amplitud más débil y de mayor duración para un pulso de radiación en la dirección opuesta (Fig. 4.17). 


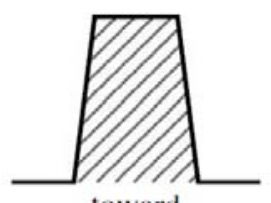

toward

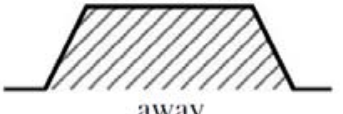

away

Figura 4.17. Pulsos en direcciones opuestas donde se aprecia la igualdad de áreas

\subsubsection{Espectro fuente.}

Consideremos ahora las características de los pulsos de campo lejano en el dominio de la frecuencia, la transformada de Fourier de una función rectangular de valor unidad en altura y anchura es:

$$
\begin{gathered}
F[B(t)]=\int_{-\frac{1}{2}}^{\frac{1}{2}} e^{i \omega t} d t=\frac{1}{i \omega}\left(e^{\frac{i \omega}{2}}-e^{-\frac{i \omega}{2}}\right)= \\
=\frac{1}{i \omega}(i \sin (\omega / 2)-i \sin (-\omega / 2)+\cos (\omega / 2)-\cos (-\omega / 2))= \\
=\frac{1}{i \omega} 2 i \sin (\omega / 2)=\frac{\sin (\omega / 2)}{\omega / 2}
\end{gathered}
$$

La función $\sin x / x$ se puede expresar comúnmente como sinc $x$. Usando el cambio de escala en la transformada de Fourier, la expresión de la función rectangular con altura unidad y anchura $\tau_{r}$ en el dominio de la frecuencia (Fig. 4.18) es:

$$
F\left[B\left(t / \tau_{r}\right)\right]=\tau_{r} \operatorname{sinc}\left(\omega \tau_{r} / 2\right)
$$
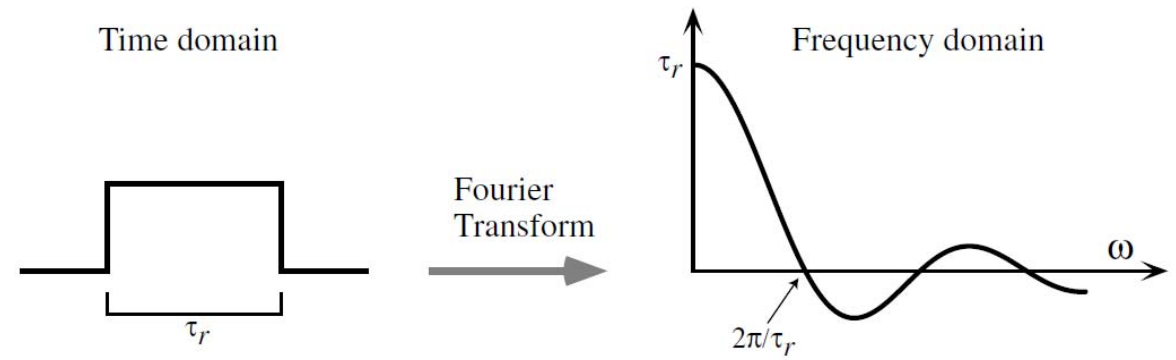

Figura 4.18. Una función rectangular en el dominio del tiempo produce una función sinc en el dominio de la frecuencia

El modelo de falla de Haskell, que viene dado por la convolución de dos funciones rectangulares de anchura $\tau_{r}$ y $\tau_{d}$, se puede expresar como el producto de dos funciones sinc en el dominio de la frecuencia:

$$
F\left[B\left(t / \tau_{r}\right) * B\left(t / \tau_{d}\right)\right]=\tau_{r} \tau_{d} \operatorname{sinc}\left(\omega \tau_{r} / 2\right) \operatorname{sinc}\left(\omega \tau_{d} / 2\right)
$$

Así, el espectro de amplitud en campo lejano para el modelo de falla de Haskell se puede expresar como: 


$$
|A(\omega)|=g M_{o}\left|\operatorname{sinc}\left(\omega \tau_{r} / 2\right)\right|\left|\operatorname{sinc}\left(\omega \tau_{d} / 2\right)\right|
$$

donde $g$ es un término escalar que incluye la dispersión geométrica ${ }^{3}$. Se suelen representar los espectros de amplitudes usando la escala logarítmica, por tanto, la expresión anterior se podría representar como

$$
\log |A(\omega)|=G+\log M_{o}+\log \left|\operatorname{sinc}\left(\omega \tau_{r} / 2\right)\right|+\log \left|\operatorname{sinc}\left(\omega \tau_{d} / 2\right)\right|
$$

donde $G=\log g$.

Si se aproxima $|\operatorname{sinc}(x)|$ a 1 para $x<1$ y a $1 / x$ para $x>1$, obtenemos la siguiente expresión

$$
\log |A(\omega)|-G=\left\{\begin{array}{cc}
\log M_{o} & \omega<2 / \tau_{d} \\
\log M_{o}-\log \frac{\tau_{d}}{2}-\log \omega & 2 / \tau_{d}<\omega<2 / \tau_{r} \\
\log M_{o}-\log \frac{\tau_{d} \tau_{r}}{4}-2 \log \omega & 2 / \tau_{r}<\omega
\end{array}\right.
$$

donde se ha asumido que $\tau_{d}>\tau_{r}$. Así se puede ver como el espectro de la función del modelo de Haskell puede ser visto (Fig. 4.19) como una parte de bajas frecuencias plana en un nivel proporcional a $M_{o}\left(\omega^{0}\right)$, un segmento proporcional a $\omega^{-1}$ en las frecuencias intermedias, y una caída proporcional a $\omega^{-2}$ en las altas frecuencias.

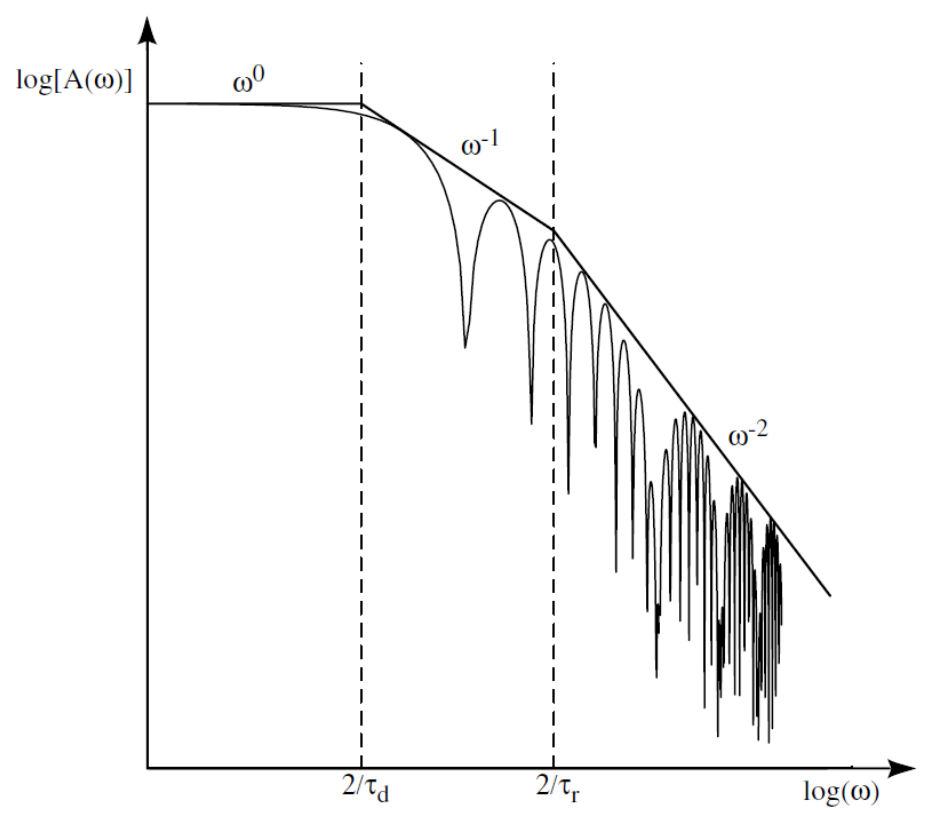

Figura 4.19. Amplitud espectral para el modelo $\omega^{-2}$ de fuente

Esto es conocido como el modelo $\omega^{-2}$ de fuente o simplemente modelo $\omega^{2}$. A las frecuencias entre $\omega=2 / \tau_{d}$ y $\omega=2 / \tau_{r}$ se las llama frecuencias de esquina, y dividen el espectro en tres partes diferentes. En el estudio de espectros de terremotos reales, se pueden obtener los valores de $M_{o}, \tau_{d}$ y $\tau_{r}$ a partir de este modelo. Sin embargo, lo más

\footnotetext{
${ }^{3}$ Atenuación debida al ensanchamiento del frente de ondas.
} 
normal es identificar solo una esquina en el espectro, la intersección de la rama $\omega^{0}$ con la rama $\omega^{-2}$, lo que se conoce como frecuencia de esquina $f_{c}$.

A la rama $\omega^{0}$ se la conoce como nivel espectral de periodos largos, $\Omega_{o}$, y como se ha dicho está relacionada a $M_{o}$ mediante la siguiente expresión:

$$
M_{o}=\frac{4 \pi \cdot \rho \cdot c^{3} \cdot r}{U_{\phi \theta}} \Omega_{o}
$$

donde $\rho$ es la densidad del terreno en la zona de rotura, $c$ es la velocidad de la onda, $r$ es la distancia hipocentral y $U_{\phi \theta}$ el patrón de radiación.

Hay que ser cuidadosos al aplicar esta interpretación del espectro de la fuente con registros reales, ya que los efectos de la atenuación y de la superficie pueden distorsionar el espectro, sobre todo en las altas frecuencias.

Muchos modelos teóricos del espectro fuente se han propuesto, pero el que más influencia ha dado es el modelo de Brune (1970), en el que el espectro de amplitud de desplazamientos viene dado por

$$
A(f)=\frac{\Omega_{o}}{1+\left(f / f_{c}\right)^{2}}
$$

donde $f_{c}$ es la frecuencia de esquina, $\omega_{c}=2 \pi f_{c}$, definida como

$$
f_{c}=4.9 \cdot 10^{6} \cdot \beta \cdot\left(\frac{\Delta \sigma}{M_{o}}\right)^{\frac{1}{3}}
$$

siendo $\beta$ la velocidad de las ondas S expresada en $\mathrm{km} / \mathrm{s}, \Delta \sigma$ es un parámetro conocido como caída de esfuerzos que se define en el apartado siguiente, y vendrá dado en bar y $M_{o}$, momento sísmico, en dinas-cm. Este modelo desarrollado por Brune está en consonancia con el modelo de fallado de Haskell, sobre todo en el decaimiento del espectro en las altas frecuencias.

\subsubsection{Caída de esfuerzos:}

La caída de esfuerzos (stress drop) se define como la diferencia media entre la tensión tangencial (tensión de cizalla) en la falla antes y después del terremoto.

$$
\Delta \sigma=\frac{1}{A} \int_{S}\left[\sigma\left(t_{2}\right)-\sigma\left(t_{1}\right)\right] d S
$$

donde se integra a lo largo de la superficie de falla y $A$ es su área. 
Considérese una falla de longitud $L$, con una anchura $w \ll L$, y un desplazamiento promedio $\bar{D}$ en la dirección de la falla. El cambio en la deformación por cortante a lo largo de la falla puede ser aproximada por $\epsilon \sim \bar{D} / w$. Como $\tau_{12}=2 \mu \epsilon_{12}$ (componente del tensor de tensiones en función de las deformaciones, donde $\mu$ es uno de los parámetros de Lamé conocido como módulo de cizalla) se puede aproximar la caída de esfuerzos para esta geometría de falla como

$$
\Delta \sigma \sim \frac{2 \mu \bar{D}}{w}
$$

Para ser más generales, también se puede expresar

$$
\Delta \sigma=C \mu\left[\frac{\bar{D}}{\tilde{L}}\right]
$$

donde $\tilde{L}$ es una dimensión característica de la ruptura ( $w$ en nuestro caso) y $C$ es una constante adimensional que depende de la geometría de la ruptura. Estas constantes geométricas solo se han desarrollado para un pequeño número de geometrías. Para el caso de una falla vertical se ha obtenido que $\tilde{L}=w / 2$ y $C=2 / \pi$.

Para estimar $\Delta \sigma$ a través de datos sísmicos, es necesario conocer las dimensiones de la falla y $\bar{D}$, el cual puede ser estimado a través del momento sísmico:

$$
\bar{D}=\frac{M_{o}}{\mu A}
$$

con lo que sustituyendo, obtenemos

$$
\Delta \sigma=\frac{C M_{o}}{A \widetilde{L}}
$$

asumiendo que $A=a \widetilde{L}^{2}$, donde $a$ es un parámetro de proporción, tenemos

$$
\Delta \sigma=\frac{C M_{o}}{a \tilde{L}^{3}}
$$

Para el caso de una ruptura circular de radio $r$, Brune en 1970 obtuvo:

$$
\Delta \sigma=\frac{7 M_{o}}{16 r^{3}}
$$

Como se puede observar, la caída de esfuerzos depende de la inversa del cubo de la longitud de la ruptura. Como esta longitud solo se puede estimar aproximadamente a partir de la duración de ruptura $\tau_{d}$ y asumiendo una velocidad de ruptura $V_{r}$, la caída de tensión es difícil de calcular con precisión por lo que requiere un ajuste. Se ha podido calcular razonablemente la caída de esfuerzos a través de la distribución de las réplicas. 
Abe (1975) obtuvo una expresión de la caída de esfuerzos relacionándola con la velocidad de ondas $\mathrm{S}$, con la densidad y la relación entre el deslizamiento final y el rise time

$$
\Delta \sigma=\beta \cdot \rho \cdot \frac{D}{\tau_{r}}
$$

y concluye estimando que para la mayoría de los terremotos, el valor de la caída de esfuerzos se encuentra entre los 10 y 100 bar, y que es independiente del momento sísmico del evento.

Por el contrario, otros estudios como Shi et al. (1998), concluyen que la caída de esfuerzos parece aumentar monótonamente con el aumento del momento sísmico de cada evento, hasta un tamaño crítico en el que se vuelve constante para eventos más grandes.

Si asumimos que un evento va a estar en consonancia con el modelo $\omega^{2}$, con la relación de Brune (1970) entre la frecuencia de esquina, momento sísmico y caída de esfuerzos, podríamos estimar del espectro de amplitudes de desplazamientos los valores de $\Omega_{o}$ y $f_{c}, \mathrm{y}$ así conseguir un valor de $\Delta \sigma$ para el evento despejando la relación de Brune

$$
\Delta \sigma=M_{o}\left(\frac{f_{c}}{4.9 \cdot 10^{6} \cdot \beta}\right)^{3}
$$

Para terremotos interplaca se obtienen valores más bajos de caída de esfuerzos que los obtenidos en terremotos intraplaca. El promedio de $\Delta \sigma$ para terremotos interplaca está sobre los 30 bares, y para intraplaca sobre los 100 bares.

\subsection{Enfoque teórico a la simulación de movimientos intensos del terreno.}

Como se dijo con anterioridad, este enfoque se basa en la aplicación de los conocimientos teóricos y modelos desarrollados para simular eventos sísmicos lo más parecido posible a la realidad. Como se dijo al explicar el teorema de representación, el movimiento intenso del terreno en un punto por la acción de una fuente sísmica a una determinada distancia es la convolución de la función fuente, que describe el proceso de ruptura en la falla, y de la función de Green, que describe los efectos del camino y del sitio hasta llegar las ondas desde el hipocentro al punto de observación. Por tanto, un enfoque teórico a las simulaciones de estos movimientos deben de tratar primero de simular los efectos de la fuente y por otro lado los efectos del camino y del sitio, para luego combinar por medio de la convolución los dos resultados y obtener un movimiento intenso del terreno lo más parecido a la realidad al que haya podido llegar. 


\subsubsection{Simulaciones teóricas de la fuente.}

\subsubsection{Modelo cinemático.}

El primer intento de simular un movimiento intenso del terreno desde el punto de vista de campo cercano lo realizó Aki (1968) para una corta distancia de 80 metros de la rotura de la falla en el terremoto de Parkfield de 1966, California. Usó para ello un modelo de fuente basado en Haskell, con un deslizamiento uniforme y una velocidad de ruptura constante sobre el plano de falla. El sismograma sintético que obtuvo se parecía en buena medida al observado.

Después, Bouchon (1979) simuló un evento para la misma estación aun más realista, teniendo en cuenta el modelo de falla Haskell dentro de una estructura de capas que simulaba la geología de la zona, usando el método discreto del número de onda, para simular la propagación.

Muchos investigadores han usado el modelo de Haskell para simular movimientos intensos del terreno para diferentes terremotos. En esos estudios obtuvieron buenos resultados acordes con los desplazamientos y con las velocidades registradas, pero no con las aceleraciones del suelo. Los registros de aceleraciones son ricos en altas frecuencias, con lo que un modelo que simula una simple dislocación no puede simularlas satisfactoriamente. La fuente sísmica es un proceso mucho más complejo que este modelo simple.

Como se apuntó al concluir el apartado referente al modelo de Haskell, se tomaron dos enfoques para solventar las deficiencias del modelo. El primero introduce la aleatoriedad en el proceso, usando los parámetros de la fuente para expresar la función de deslizamiento estocásticamente. El otro enfoque toma la función de deslizamiento y la propagación de la ruptura como variaciones irregulares sobre el plano de falla en sentido determinista.

Mikumo y Miyatake (1978) tomaron el enfoque estocástico de la falla asumiendo que los parámetros como desplazamientos, velocidad de deslizamiento, ángulos de deslizamiento, y velocidades de ruptura, son funciones de la posición sobre el plano de falla y que se distribuyen aleatoriamente con una variación respecto de su media según la desviación estándar. Con este modelo consiguieron generar adecuados movimientos de altas frecuencias pero no así conseguir una simulación correcta de los movimientos fuertes, debido a la falta de base física para dar aleatoriedad a los parámetros del modelo de falla.

En 1983, Papageorgiou y Aki propusieron un modelo hibrido determinista-estocástico de falla, conocido como modelo de barrera específico, para generar movimientos fuertes del terreno derivados de un escalado del espectro fuente. Este modelo idealiza el plano de falla como rectangular, y asume que un terremoto grande puede ser modelizado como pequeñas subfallas circulares distribuidas uniformemente en la superficie rectangular del plano de falla, como se puede ver en la Fig. 4.20. 
A escala macroscópica, la ruptura se propaga a lo largo de el plano de falla con una velocidad de ruptura promedio, $V_{r}$. A escala microscópica, dentro de cada subfalla se inicia la rotura en el centro y se extiende radialmente a los bordes.

La función temporal fuente para cada subfalla fue dada por Sato y Hirasawa (1973) basándose en un modelo cinemático con una distribución de deslizamientos de la solución estática de una ruptura circular.

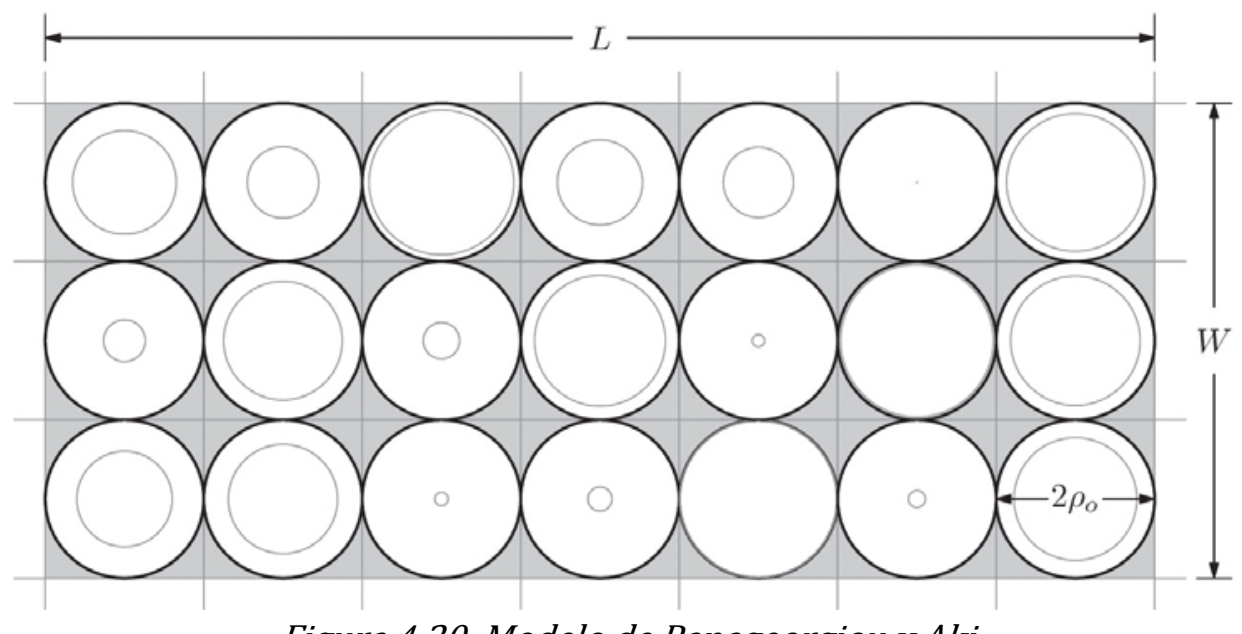

Figura 4.20. Modelo de Papageorgiou y Aki

Para realizar una simulación con este método, además de conocer los parámetros globales de la falla, es necesario conocer parámetros como el tamaño de las subfallas, la caída de esfuerzos locales y el tamaño de la zona cohesiva.

Suzuki y Hirasawa (1984) propusieron un método similar de modelo de falla estocástico basado en la cinemática con tamaños aleatorios de subfallas cuadradas en un plano de falla mayor. Consideraron que el contenido de altas frecuencias de los movimientos se generaba por distribuciones de resistencia de la falla no homogéneas. Desde un punto de vista sismológico, el problema de este modelo es la existencia de demasiados parámetros a conocer y de la selección de variables aleatorias las cuales tienen un significado físico confuso.

Otros desarrollos para los movimientos de altas frecuencias fueron, por ejemplo, Trifunac y Udiwadia (1974) que para el terremoto de Parkfield tomaron un deslizamiento no uniforme en el plano de falla. Como los movimientos pueden ser detectados por numerosas estaciones, se pueden desarrollar métodos que nos den la distribución de deslizamientos en el plano de falla a través del estudio del problema inverso a partir de los registros.

Muchos estudios se han realizado sobre este problema inverso, lo que ha llevado a ideas como las asperezas y las barreras para comprender el proceso de ruptura en los terremotos. Con esto se intenta comprender el proceso dinámico en la fuente sobre la base de los resultados del problema inverso utilizando el modelo cinemático. 


\subsubsection{Modelo dinámico.}

La otra manera de ver el problema de simular la fuente es usando un modelo dinámico de la fuente, construyendo la fuente sísmica no solo satisfaciendo la ruptura por cizalla cinemáticamente sino a través de la compresión de las condiciones de esfuerzos en el plano de falla de manera dinámica.

El pionero en realizar un modelo dinámico de la fuente fue Kostrov (1966), que resolvió analíticamente la propagación de la ruptura por cortante en un antiplano bidimensional a una velocidad de ruptura fija y demostró el comportamiento de la velocidad de deslizamiento y de la tensión cerca de la punta de la grieta.

Usando el método de las diferencias finitas, Madariaga (1976) calculó el movimiento sísmico para campo lejano y el espectro para una ruptura circular con una velocidad de ruptura constante y la parada de la misma para un determinado radio. Los resultados muestran que las fases de parada dominan las altas frecuencias.

Los movimientos en campo lejano obtenidos por Madariaga por medio del modelo de rotura dinámico son similares a los que obtuvieron Sato y Hirasawa (1973), mencionados en el apartado anterior, los cuales asumieron que la solución estática conocida tiene en cada instante sucesivo a la formación de la ruptura circular un esfuerzo de cortante uniforme.

Al considerar una propagación espontánea de la ruptura, necesitamos tener algunos criterios básicos de mecánica de la fractura, como los criterios de Griffinth y de Irwin.

- El criterio de Griffinth establece que la fractura se extiende cuando el suministro de energía mecánica desde el medio circundante excede la energía superficial consumida para un aumento infinitesimal virtual de la longitud de la grieta.

- El criterio de Irwin establece que la fractura se extiende cuando el factor intensidad - tensión de la punta de la grieta excede un valor crítico que es una constante del material.

Hamano (1974) mostró que el criterio de Irwin correspondía al nivel de esfuerzo crítico que se promedia sobre cada cuadrícula en la que se divide el plano de falla. Este concepto es muy útil para simulaciones numéricas de nucleación y de parada del proceso de ruptura. Das y Aki (1977) demostraron la validez del criterio de Hamano numéricamente usando el método de las diferencias finitas, y calculando el movimiento del terreno y el espectro radiado por una grieta finita que se propaga espontáneamente y luego se para. Basado en estos resultados, Das y Aki propusieron el modelo de barrera (barrier model) mostrando que la presencia de barreras sin fracturar pueden generar contenidos de movimientos en las altas frecuencias.

Físicamente, es necesario conocer la velocidad de deslizamiento y la tensión alrededor de la punta de la grieta que se propaga, y pueden ser modeladas introduciendo una fuerza cohesiva, la cual se distribuye en la grieta cerca de la punta y se opone a las tensiones 
exteriores. El tamaño de la zona cohesiva dividido por la velocidad de ruptura, puede controlar el extremo más alto de la frecuencia del espectro y el límite de altas frecuencias en la frecuencia de esquina del espectro de aceleración $\left(f_{\max }\right)$. Este controlador puede ser muy importante en la descripción de las características de la fuente.

Usando el método de los elementos de contorno, Fujiwara y Irikura (1991) realizaron simulaciones de desplazamientos, velocidad y aceleración cerca de la grieta, usando el criterio de Griffith con un modelo de zona cohesiva.

Los criterios de mecánica de la fractura son importantes para describir físicamente las fuentes sísmicas por medio de un modelo dinámico, aunque es muy complicado el modelado y no siempre son útiles para realizar predicciones o simulaciones en el campo cercano porque necesitan gran cantidad de información sobre los parámetros físicos como la tensión y resistencia, y su distribución sobre el plano de falla.

Debidos a estos problemas, se desarrollaron métodos híbridos de los modelos cinemático y dinámico para generar ondas de altas frecuencias, como por ejemplo Sato (1984) con su propuesta de ruptura irregular. Para Sato el plano de falla para un terremoto de tamaño importante se divide en pequeñas subfallas y el movimiento intenso generado por cada subfalla es tomado como una fuente puntual. El movimiento intenso total será la superposición de las ondas sísmicas generadas por las fuentes puntuales. Los parámetros de la falla como el desplazamiento final, el rise time y la velocidad de ruptura vienen dados como la suma de los parámetros homogeneizados para cada subfalla.

La función temporal de la fuente para cada subfalla viene dada como una función tipo rampa escalonada con varios saltos que representan deslizamientos, generados aleatoriamente, basados en los experimentos de laboratorio realizados por Ohnaka (1986).

\subsubsection{Simulación teórica de los efectos del camino y del sitio.}

Para realizar la simulación con un enfoque teórico de los efectos del camino y del sitio es necesario calcular una función de Green plausible con las estructuras del terreno desde la fuente hasta el receptor.

Los métodos para resolver directamente los sistemas de ecuaciones diferenciales para los problemas elastodinámicos como este son el método de las diferencias finitas, el método de los elementos finitos, o el método de los elementos de contorno. Aunque tengan un gran poder de cálculo, modelar la estructura del terreno es una labor muy dura que necesitará ser simplificada dadas las limitaciones de computación que conlleva representar el terreno.

Una de las simplificaciones más usadas es la de tomar el terreno como heterogéneo verticalmente, con lo que el medio se puede tomar como un paquete de capas homogéneas e isotrópicas en horizontal. Con esto se consigue simplificar bastante la obtención de una función de Green para este modelo del terreno, tanto en dos dimensiones como en tres. 
Un método práctico de estimar los efectos del camino y del sitio es la de separar los términos de la fuente de los registros observados en un número importante de estaciones para un mismo evento (Andrews (1986), Iwata and Irikura (1988)).

La simulación teórica de los efectos del camino y del sitio, o lo que es lo mismo, el desarrollo de una función de Green teórica, no deja de ser un trabajo muy laborioso, y del cual solo se puede conseguir el objetivo tras incurrir en simplificaciones varias.

\subsection{Enfoque semiempírico de la simulación de movimientos intensos.}

Uno de los métodos más efectivos para simular movimientos fuertes del terreno que provengan de un terremoto de una intensidad considerable para una zona determinada, es usando los registros de eventos más pequeños, observados en esa misma zona.

La estructura geológica desde la fuente sísmica al punto de observación es de una gran complejidad como para poder asumir modelos teóricos como los descritos someramente en el apartado anterior. Los movimientos fuertes del terreno no solo son complejos por la refracción y reflexión de las ondas debido a la presencia de capas y estructuras del terreno, sino también a la atenuación y la dispersión debidas a las heterogeneidades laterales y las propiedades anelásticas en el camino de propagación. Un modelado completo del comportamiento de las ondas en un medio realista es extremadamente complejo y muy costoso como para ser de utilidad a la hora de predecir o simular eventos sísmicos en una determinada zona.

Con el enfoque semiempírico, se intentan solventar estas dificultades usando como función de Green empírica el registro del evento menor y describiendo una función fuente como una función tiempo fuente equivalente que, como desarrollaremos en los siguientes capítulos de este documento, se podrá modelar de manera puramente estadística o mediante un modelado cinemático de la ruptura.

Matemáticamente esto se puede expresar de la siguiente manera:

$$
S(t)=\operatorname{ESTF}(t) * S(t)
$$

es decir, que el registro sintético para un evento sísmico mayor registrado en un determinado punto de observación, $S(t)$, se obtiene de la convolución de la función temporal equivalente de la fuente, $\operatorname{ESTF}(t)$, con el registro de eventos más pequeños registrados en ese mismo punto de observación y con hipocentro en la misma zona que el supuesto evento mayor.

La función temporal equivalente de la fuente, tanto para el modelado estadístico, como para el cinemático, tiene la siguiente forma

$$
\operatorname{ESTF}(t)=\kappa \cdot \sum \delta\left(t-t_{i}\right)
$$


es decir, el producto de una constante que multiplica a la sumatoria de un determinado número de funciones impulsivas o delta de Dirac con unos determinados retardos. La obtención de estos tiempos de retardo y de los parámetros que gobiernan esta función fuente sintética es lo que diferencia sustancialmente los dos tipos de modelado.

La idea del uso de registros de eventos menores como funciones de Green empíricas fue originalmente introducida por Hartzell (1978), que simuló terremotos mayores usando los registros de réplicas como funciones de Green en lugar de funciones de Green teóricas, aunque no tuvo en cuenta las relaciones de escala entre los eventos mayor y menores.

Kanamori (1979) extendió el método para reproducir los movimientos del terreno provocados por un terremoto histórico con una secuencia de eventos medianos, los cuales sumó con unos intervalos aleatorios de manera que conseguía el momento sísmico del evento objetivo. Comparando el contenido espectral de la simulación, con el modelo $\omega^{2}$, la suma sobreestimaba el contenido en altas frecuencias, mientras que era acertada para las bajas frecuencias.

A partir de estas ideas principales los investigadores comenzaron a desarrollar los distintos modelos sobre los que aplicar el concepto de función de Green empírica usando registros de eventos más pequeños. Con ello se consigue meter en la simulación los efectos de camino y de sitio, tan difíciles de simular con el enfoque teórico, y solo hay que preocuparse de modelizar correctamente la función fuente.

Importante para este método es el de comparar los espectros de desplazamientos con el modelo $\omega^{2}$, ya que nos da unas relaciones de escala y así relacionar los eventos menores con los mayores y viceversa.

\subsubsection{Relaciones de escala en la fuente.}

Aunque no tan claramente como en el modelado cinemático, los parámetros de escalado del modelado estadístico se pueden relacionar con las siguientes relaciones de escala que se obtuvieron principalmente para el modelo de fuente determinista cinemático.

Tomemos un modelo de falla como el expresado en la sección de fundamentos sismológicos, en el cual el plano de la falla lo representábamos como un rectángulo, y que, por el principio de superposición lineal, una ruptura de falla mayor podía representarse como la suma de la respuesta de un número limitado de fallas más pequeñas, y que también consideremos estas rectangulares.

La fuente sísmica se caracteriza, como se dijo también en ese apartado de fundamentos, a través de cinco parámetros:

- Longitud de la falla, $L$.

- Anchura de la falla, $W$.

- Desplazamiento final, $D$.

- Rise time, $T$.

- Velocidad de ruptura, $V_{r}$. 
El escalado de los parámetros de la fuente, como el área de la falla $(L x W)$, el deslizamiento, la duración del deslizamiento y la velocidad de ruptura, respecto del tamaño de la fuente del terremoto fue estudiado por Kanamori y Anderson (1975) como un similaridad empírica entre terremotos (Fig. 4.21). La relación de escalado que obtuvieron fue

$$
\frac{L}{l}=\frac{W}{W}=\frac{D}{d}=\frac{T}{\tau}=N
$$

donde $L$ y $l$ son la longitud de la falla para un evento mayor y otro menor, $W$ y $w$ las anchuras de las respectivas fallas, $D$ y $d$ los desplazamientos finales, y $T$ y $\tau$ los respectivos rise time.

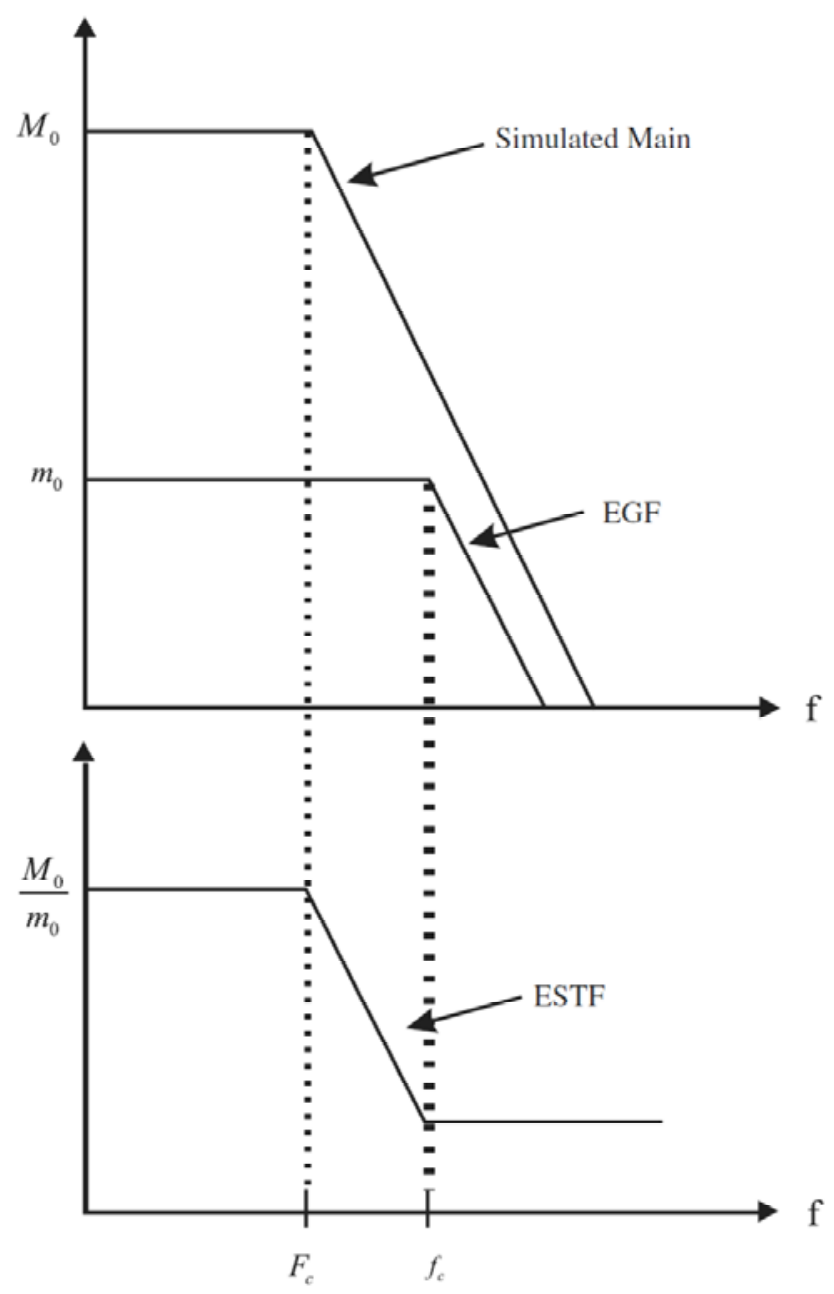

Figura 4.21. Relación entre los espectros de desplazamiento del evento objetivo y el evento función de Green empírica (EGF)

Con estas relaciones anteriores, obtenemos un escalado de "geometría", pero si además comparamos el contenido espectral para espectros de desplazamiento del evento mayor y del menor, y además partimos de la base de que obedecen al modelo $\omega^{2}$ de fuente, como podemos ver la imagen anterior (Fig. 4.21), aparecen otras relaciones. 
Sean $M_{o s}$ y $f_{c S}$ el momento sísmico y la frecuencia de esquina del evento pequeño o función de Green empírica, y $M_{o o}$ y $f_{c o}$, el momento sísmico y la frecuencia de esquina del evento objetivo a simular. Usando la expresión de Brune (1970) enunciada en apartados anteriores para la frecuencia de esquina

$$
f_{c}=4.9 \cdot 10^{6} \cdot \beta \cdot\left(\frac{\Delta \sigma}{M_{o}}\right)^{\frac{1}{3}}
$$

con $\omega_{c}=2 \pi f_{c}$, y siendo $\beta$ la velocidad de las ondas S expresada en $\mathrm{km} / \mathrm{s}, \Delta \sigma$ vendrá dada en bar y $M_{o}$ en dina-cm.

Y si además, usamos las relaciones

$$
\frac{\Delta \sigma_{o}}{\Delta \sigma_{s}}=C
$$

donde $\Delta \sigma_{o}$ y $\Delta \sigma_{s}$ son las caídas de esfuerzos del evento objetivo y del evento pequeño respectivamente, y la relación

$$
\frac{M_{o o}}{M_{o s}}=C N^{3}
$$

que relaciona los momentos sísmicos de los dos eventos, podemos relacionar la frecuencia de esquina del evento objetivo con la del evento función de Green empírica de la siguiente manera

$$
\frac{f_{c o}}{f_{c S}}=\frac{4.9 \cdot 10^{6} \cdot \beta \cdot\left(\frac{\Delta \sigma_{o}}{M_{o o}}\right)^{\frac{1}{3}}}{4.9 \cdot 10^{6} \cdot \beta \cdot\left(\frac{\Delta \sigma_{s}}{M_{o S}}\right)^{\frac{1}{3}}}=\left(\frac{M_{o o}}{M_{o S}}\right)^{-\frac{1}{3}} \cdot\left(\frac{\Delta \sigma_{o}}{\Delta \sigma_{S}}\right)^{\frac{1}{3}}=\left(\frac{M_{o o}}{C \cdot M_{o s}}\right)^{-\frac{1}{3}}=1 / N
$$

por tanto se puede expresar que

$$
\begin{gathered}
N=\frac{f_{c S}}{f_{c o}} \\
C=\frac{M_{o o}}{M_{o s}} \cdot N^{-3}
\end{gathered}
$$

Con las relaciones anteriores vemos que podemos relacionar todos los parámetros que caracterizan la fuente sísmica. Y además si nos fijamos en la expresión de Brune de la frecuencia de esquina, conociendo la $f_{c}$ y el momento sísmico, que se calculan fácilmente a través del espectro de desplazamientos, podemos obtener la caída de esfuerzos para el evento determinado solo despejando su valor. 


\section{ENFOQUE SEMIEMPÍRICO. MODELADO ESTADÍSTICO.}

A partir del registro de un evento pequeño, obtenido en el sitio de interés donde se quiere realizar la simulación, del momento sísmico, $M_{o s}, \mathrm{y}$ de la frecuencia de esquina, $f_{c S}$, podemos desarrollar un modelado estadístico del método de la función de Green empírica.

El objetivo de estos métodos es el de sintetizar un registro con un momento sísmico, $M_{o o}$, y una frecuencia de esquina, $f_{c o}$, fijados de antemano que ocurra en la misma región que el evento pequeño y que tenga el mismo mecanismo focal. Por tanto aunque no es estrictamente verdad, estamos tomando el registro del evento menor como función de Green para todos los puntos del área de ruptura del evento objetivo.

En el modelado estadístico que desarrollamos a continuación se considera que el registro sintético tendrá el mismo mecanismo focal que el terremoto pequeño, aunque no se utiliza explícitamente este dato para su modelado.

Como en todos los métodos de simulación basados en el enfoque semiempírico, se asume que el espectro fuente de los registros función de Green empírica (EGF), como en los registros simulados, obedecen a un comportamiento similar (la ley $\omega^{2}$ ) que se ha explicado en apartados anteriores, desarrollada por Aki (1967) y Brune (1970).

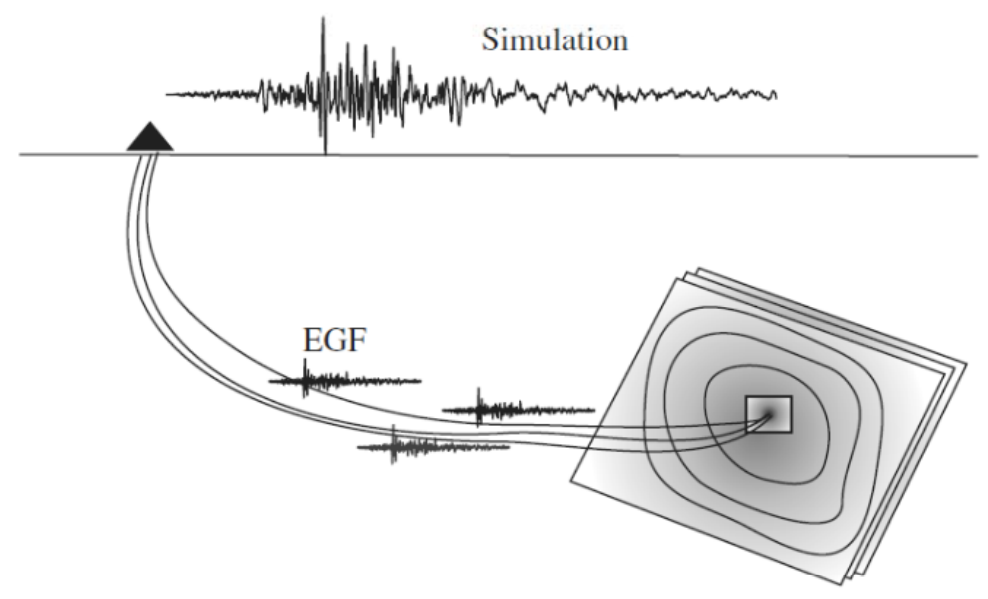

Figura 5.1. Síntesis de un modelo basado en el uso de la función de Green Empírica

La imagen anterior (Fig. 5.1) muestra esquemáticamente el principio de los métodos semiempíricos basados en el modelado estadístico. A partir del registro de un evento sísmico menor, se combina un número determinado de veces para producir un registro simulado de un evento mayor registrado en la misma estación. Este número determinado de veces queda definido por las relaciones de escala desarrolladas en apartados anteriores 
y su distribución temporal está determinada para este tipo de modelado por una función de densidad de probabilidad, $p(t)$. Esta función de densidad de probabilidad es la que gobierna cada uno de los modelados estadísticos.

Muchos son los autores que han trabajado en este campo, desarrollando este método de suma estocástica, Joyner y Boore (1986), Wennerberg (1990), Somerville (1993), Turkimarkin y Archuletta (1994), Zeng et al (1994), Ordaz, Singh y Arboleda (1995), Kohrs-Sonsory et al (2005), Di Alessandro y Boatwright (2006), Courboulex et al (2010). A rasgos generales estos trabajos se podrían clasificar en dos grandes subgrupos, los que desarrollan métodos de suma estocástica simple como Wennerber y Ordaz et al. y los que desarrollan métodos de dos pasos, con lo que tienen que usar dos funciones de densidad de probabilidad, como Kohrs-Sonsory y Courboulex.

A continuación se desarrollará el modelo desarrollado por Ordaz, Singh y Arboleda en 1995, (en adelante método OSA), que será el que se utilizará como método estadístico en este trabajo.

\subsection{Método O.S.A.}

Este método está basado en el enfoque de Wennerber, obteniendo una función de densidad de probabilidad de los tiempos de retardo, que es consecuente con el procedimiento original de Joyner and Boore. Tomando como punto de partida la expresión general de los métodos EGF

$$
\begin{gathered}
S(t)=\operatorname{ESTF}(t) * s(t) \\
\operatorname{ESTF}(t)=\kappa \cdot \sum \delta\left(t-t_{i}\right)
\end{gathered}
$$

siendo como ya se ha explicado en el apartado anterior, $s(t)$ el registro del evento pequeño (se denota por $s(t)$ ya que puede ser aceleración, $a(t)$, velocidad, $v(t)$, o desplazamientos, $u(t)$, por la propiedad de derivación de la convolución), $S(t)$ el registro sintético del evento objetivo, $\operatorname{ESTF}(t)$ la función temporal equivalente de la fuente (del inglés "equivalent source time function"), $\kappa$ un parámetro relacionado con las relaciones de escala y $\sum \delta\left(t-t_{i}\right)$ una suma de funciones impulso o delta de Dirac, con unos determinados retardos.

En Ordaz et al. (1995), se determina que la función temporal equivalente de la fuente viene dada por

$$
\operatorname{ESTF}(t)=\xi \cdot \sum_{j=1}^{\eta} \delta\left(t-t_{j}\right)
$$


Los tiempos de retardo $t_{j}$ son aleatorios, independientes y igualmente distribuidos con la función densidad de probabilidad $p(t)$. Si aplicamos la transformada de Fourier, la formulación anterior en el domino de la frecuencia será

$$
\begin{aligned}
& S(\omega)=\operatorname{ESTF}(\omega) \cdot s(\omega) \\
& \operatorname{ESTF}(\omega)=\xi \cdot \sum_{j=1}^{\eta} e^{-i \omega t_{j}}
\end{aligned}
$$

El valor esperado, es decir, la esperanza, de $|S(\omega)|^{2}, E\left[|S(\omega)|^{2}\right]$, vendrá dada por

$$
E\left[|S(\omega)|^{2}\right]=\xi^{2} \cdot|s(\omega)|^{2} \cdot\left[\eta+\left(\eta^{2}-\eta\right) \cdot|P(\omega)|^{2}\right]
$$

donde $P(\omega)$ es la transformada de Fourier de $p(t)$. La razón espectral esperada entre la señal simulada y la señal original, $R(\omega)$, se puede estimar como

$$
R(\omega)=\sqrt{\frac{E\left[|S(\omega)|^{2}\right]}{|s(\omega)|^{2}}} \approx \xi \cdot \sqrt{\left[\eta+\left(\eta^{2}-\eta\right) \cdot|P(\omega)|^{2}\right]}
$$

Por definición, $P(0)=1$, por lo que $R(0)=\xi \cdot \eta$. Por otro lado, si requerimos que $|P(\omega)|$ decaiga como $\omega \rightarrow \infty$, entonces $R(\infty)=\xi \cdot \eta^{1 / 2}$.

Como se ha dicho antes, estamos asumiendo que las fuentes del evento pequeño como del evento grande siguen el modelo $\omega^{2}$, lo cual implica que la razón espectral entre el evento objetivo y el evento menor, $H(\omega)$, debe ser

$$
H(\omega)=\frac{M_{o o}}{M_{o s}}\left[\frac{1+\left(\frac{\omega}{\omega_{c s}}\right)^{2}}{1+\left(\frac{\omega}{\omega_{c o}}\right)^{2}}\right]
$$

donde $\omega_{c}$ es la frecuencia de esquina y $M_{o}$ el momentos sísmico de los respectivos eventos.

Relacionando los dos ratios, obtenemos los valores de $\xi$ y $\eta$

$$
\begin{aligned}
& \eta=\left(\frac{M_{o o}}{M_{o s}}\right)^{\frac{4}{3}} \cdot\left(\frac{\Delta \sigma_{o}}{\Delta \sigma_{s}}\right)^{-\frac{4}{3}} \\
& \xi=\left(\frac{M_{o o}}{M_{o s}}\right)^{-\frac{1}{3}} \cdot\left(\frac{\Delta \sigma_{o}}{\Delta \sigma_{S}}\right)^{\frac{4}{3}}
\end{aligned}
$$

donde $\Delta \sigma$ es la caída de esfuerzos, relacionado con la frecuencia de esquina por la expresión de Brune (1970) enunciada en apartados anteriores,

$$
\omega_{c}=2 \pi f_{c}
$$




$$
f_{c}=4.9 \cdot 10^{6} \cdot \beta \cdot\left(\frac{\Delta \sigma}{M_{o}}\right)^{\frac{1}{3}}
$$

siendo $\beta$ la velocidad de las ondas S expresada en $\mathrm{km} / \mathrm{s}, \Delta \sigma$ vendrá dada en bar y $M_{o}$ en dina-cm.

Se puede encontrar una relación entre los parámetros $\xi$ y $\eta$, y las relaciones de escala generales para los métodos basados en la función de Green empírica. Desarrollando e igualando expresiones, llegamos a

$$
\begin{gathered}
\eta=\left(\frac{M_{o o}}{M_{o s}}\right)^{\frac{4}{3}} \cdot\left(\frac{\Delta \sigma_{o}}{\Delta \sigma_{S}}\right)^{-\frac{4}{3}}=N^{4} \\
\xi=\left(\frac{M_{o o}}{M_{o S}}\right)^{-\frac{1}{3}} \cdot\left(\frac{\Delta \sigma_{o}}{\Delta \sigma_{S}}\right)^{\frac{4}{3}}=\frac{C}{N}
\end{gathered}
$$

Recordemos que

$$
\begin{gathered}
N=\frac{f_{c S}}{f_{c o}} \\
C=\frac{\Delta \sigma_{o}}{\Delta \sigma_{S}}=\frac{M_{o o}}{M_{o s}} \cdot N^{-3}
\end{gathered}
$$

para tener una referencia de las relaciones que estamos manejando.

Con los valores de $\xi$ y $\eta$ se consigue un correcto escalado tanto para las frecuencias muy bajas como para las muy altas. Para las frecuencias intermedias $R(\omega)$ depende de $P(\omega)$ y por tanto de la función de densidad de probabilidad de los tiempos de retardo, $p(t)$. Si se requiere que la escala se mantenga para todas las frecuencias, $H(\omega)$ debe de ser igual a $R(\omega)$ para todas las frecuencias, por tanto igualando para todos los valores de $\omega$ se obtiene

$$
|P(\omega)|=\frac{\sqrt{1+\alpha \cdot\left(\frac{\omega}{\omega_{c o}}\right)^{2}}}{1+\left(\frac{\omega}{\omega_{c o}}\right)^{2}}
$$

siendo $\alpha$

$$
\alpha=\frac{2 \omega_{c o}^{2}}{\omega_{c o}^{2}+\omega_{c s}^{2}}
$$

Siguiendo a Wennerberg (1990), Ordaz et al. (1995) imponen la restricción adicional de que $P(\omega)$ sea un número real. Con esta restricción se puede aplicar la transformada inversa de Fourier con lo que tendremos la expresión de la función de densidad de probabilidad en el dominio del tiempo (Fig. 5.2) tal que 


$$
p(t)=\frac{1}{2 \pi} \int_{-\infty}^{+\infty} \frac{\sqrt{1+\alpha \cdot\left(\frac{\omega}{\omega_{c o}}\right)^{2}}}{1+\left(\frac{\omega}{\omega_{c o}}\right)^{2}} e^{i \omega t} d \omega
$$
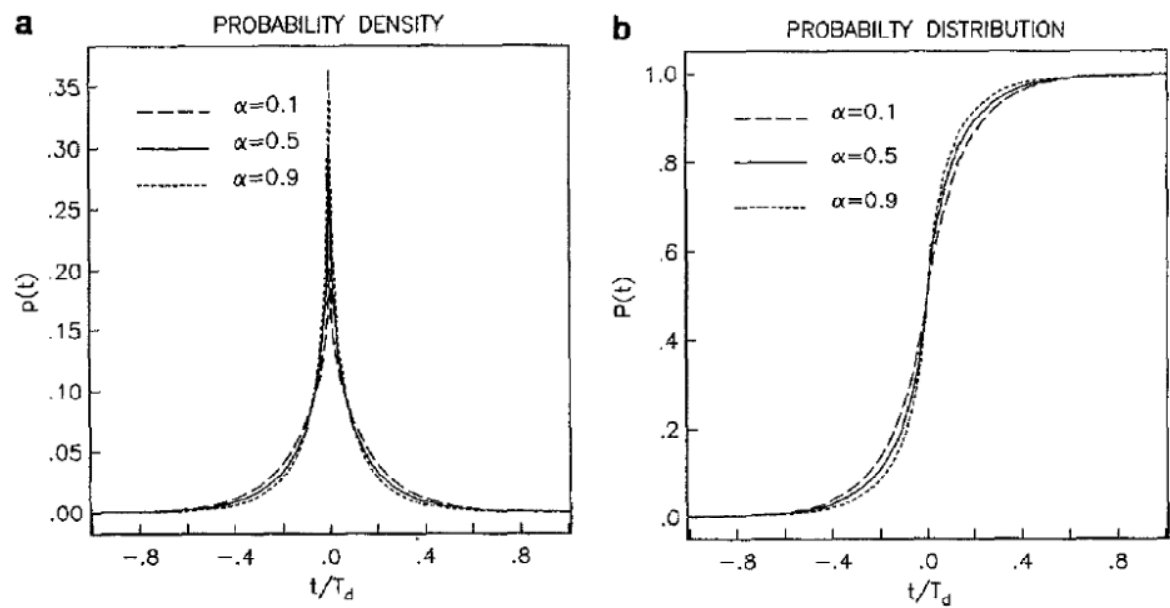

Figura 5.2. Funciones de densidad de probabilidad y de distribución de densidad del Método O.S.A.

Hay que tener en cuenta que $p(t)$ se extiende desde $-\infty$ a $+\infty$, por lo que los tiempos de retardo simulados pueden ser negativos. Además, hay una probabilidad distinta de cero de que el tiempo total de ruptura simulado sea mayor que la duración de la ruptura $T_{d} \approx 2 \pi / \omega_{c o}$ (Hanks y McGuire, 1981), pero para cualquier simulación, el número de subeventos que ocurren fuera de la duración de ruptura esperada es muy pequeño, como se puede ver en la imagen anterior (Fig. 5.2).

\subsection{Implementación del método O.S.A.:}

\subsubsection{Datos del medio y de la función de Green empírica.}

El medio solo tenemos que caracterizarlo por la velocidad de las ondas $S, V_{S}$

Los datos principales para definir la función de Green empírica son:

- Registros en las tres direcciones del espacio. Normalmente acelerogramas o sismogramas con una determinada frecuencia de muestreo, la cual también será para las simulaciones. 
- Magnitud momento y frecuencia de esquina.

El momento sísmico asociado al evento EGF lo obtenemos de la magnitud momento despejando de la expresión de Kanamori

$$
M_{w}=\frac{2}{3} \log _{10} M_{o}-10.7
$$

Conociendo el momento sísmico y la frecuencia de esquina, podemos determinar la caída de esfuerzos, si el espectro de desplazamientos cumple el modelo de Brune, a través de la expresión

$$
\Delta \sigma=M_{o}\left(\frac{f_{c}}{4.9 \cdot 10^{6} \cdot \beta}\right)^{3}
$$

donde $\beta$ es la velocidad de las ondas S expresada en $\mathrm{km} / \mathrm{s}, \Delta \sigma$ está en bar y $M_{o}$, momento sísmico, en dina-cm.

\subsubsection{Datos del evento objetivo}

Para el evento objetivo a calcular se fijan los siguientes datos

- Magnitud momento, frecuencia de esquina y caída de esfuerzos, de la misma manera que se han calculado para el evento menor o evento función de Green empírica.

- Se calculan los parámetros de escalado $C, \eta$ y $\xi$

$$
\begin{gathered}
\frac{\Delta \sigma_{o}}{\Delta \sigma_{s}}=C \\
\eta=\left(\frac{M_{o o}}{M_{o s}}\right)^{\frac{4}{3}} \cdot\left(\frac{\Delta \sigma_{o}}{\Delta \sigma_{s}}\right)^{-\frac{4}{3}} \\
\xi=\left(\frac{M_{o o}}{M_{o s}}\right)^{-\frac{1}{3}} \cdot\left(\frac{\Delta \sigma_{o}}{\Delta \sigma_{s}}\right)^{\frac{4}{3}}
\end{gathered}
$$

\subsubsection{Obtención de la función temporal equivalente de la fuente.}

Para obtener los tiempos de retardo necesarios para la obtención de la función temporal equivalente de la fuente, es necesario obtener números aleatorios con la función de densidad de probabilidad $p(t)$, determinada en el apartado anterior. 
La manera más simple de conseguir este objetivo es usando el método inverso, en el cual un número con una distribución de probabilidad $P(t)$ puede ser obtenida a través de la relación

$$
t_{i}=P^{-1}\left(u_{i}\right)
$$

donde $t_{i}$ es un número aleatorio con la probabilidad deseada, $u_{i}$ es un número aleatorio con una distribución de probabilidad uniforme entre 0 y 1 , y $P^{-1}(u)$ es la función inversa de la función de distribución de probabilidad, que por definición, está relacionada con la función de densidad de probabilidad $p(t)$.

$$
P(t)=\int_{-\infty}^{t} p(\tau) d \tau
$$

La aplicación del método inverso requiere que $P(t)$ pueda ser invertible.

Ordaz, Singh y Arboleda no encontraron una expresión analítica para la doble integración requerida para dar una expresión para $P(t)$. No obstante, se puede utilizar una integración numérica ya que se produce una convergencia hacia la solución rápidamente. La expresión a usar es

$$
P(t)=\frac{1}{2}+\frac{1}{2}\left(1-e^{-\omega_{c o} t}\right) \sqrt{1-\alpha}+\frac{1}{\pi} \sqrt{\alpha} \int_{1 / \sqrt{\alpha}}^{+\infty} \frac{\sqrt{y^{2}-\frac{1}{\alpha}}}{y\left(y^{2}-1\right)}\left(1-e^{-\omega_{c o} t y}\right) d y
$$

para $t \geq 0$.

Como la función de densidad de probabilidad es simétrica respecto $t=0$, se puede ver que la función distribución de probabilidad tiene la propiedad siguiente

$$
P(-t)=1-P(t)
$$

Una vez que $P(t)$ se puede evaluar, su función inversa se consigue resolviendo numéricamente la expresión $u=P(t)$ de manera iterativa para valores de $u$ comprendidos entre 0 y 1 . Dada la cantidad de cálculos la mejor opción es la de construir una tabla de pares $\left(u_{i} ; t_{i}\right)$ para valores de $u$ igualmente espaciados y suficientemente cercanos como para que las aproximaciones no cometan demasiados errores.

Obteniendo los $\eta$ valores de los $u$ números aleatorios necesarios para generar la función tiempo fuente equivalente, solo hay que ir a la tabla, buscar los $\left(u_{i} ; t_{i}\right)$ superior e inferior y se realiza la interpolación para obtener los $\eta$ valores de tiempo de retardo.

A la hora de implementar este problema con el tiempo de duración de la ruptura, se determinará que sea el valor mayor entre la expresión anterior de Hanks y McGuire o el tiempo que tenga una probabilidad de ocurrencia del 95\%. 
Una vez determinada la función temporal equivalente de la fuente, con la expresión

$$
\operatorname{ESTF}(t)=\xi \cdot \sum_{j=1}^{\eta} \delta\left(t-t_{j}\right)
$$

Se realiza la convolución y la simulación de la siguiente manera

$$
S(t)=\operatorname{ESTF}(t) * S(t)
$$

obteniendo una simulación a partir del modelado estadístico.

\subsection{Simulaciones con el método O.S.A.}

Los autores del método realizaron muchas simulaciones con muy buenos resultados respecto a eventos mayores registrados, como podemos ver en Ordaz et al. (1995). Por lo que él método podemos usarlo directamente para realizar nuestras simulaciones, ya que se ajusta perfectamente a la formulación original. No obstante mostraremos algunos casos para ver los resultados que se obtienen al usar esta implementación del método de Ordaz, Singh y Arboleda.

Tomando el terremoto del 5 de Enero de 2007 de Sierra Elvira, que se puede ver en el Anejo 1, con estación de registro denominada Estación 1, en la Facultad de Ciencias de la Universidad de Granada. Realizamos dos simulaciones con este modelo estadístico, una con magnitud momento esperada $6.0 \mathrm{y}$ una frecuencia de esquina esperada de $0.25 \mathrm{~Hz}$, y otra de magnitud momento esperada de 6.9 y una frecuencia de esquina de $0.06 \mathrm{~Hz}$.

Lo que se busca es, fijando un mismo evento función de Green empírica, ver como varían los valores de los parámetros inherentes al modelado estadístico para distintos casos de magnitud momento esperados y de frecuencia de esquina. Para el primer caso tenemos los resultados de la Tabla 5.1.

\begin{tabular}{|c|c|c|c|}
\hline Datos del Medio & & & \\
\hline Velocidad de Ondas S & \multicolumn{2}{|r|}{3.5} & \\
\hline Velocidad de Ruptura & \multicolumn{2}{|c|}{2.975} & \\
\hline Datos de los Eventos & Evento EGF & Evento Objetivo & \\
\hline Magnitud Momento & 4.1 & 6 & \\
\hline Momento Sísmico & $1.58 \mathrm{E}+15$ & $1.12 \mathrm{E}+18$ & \\
\hline Frecuencia de Esquina & 1.4 & 0.25 & \\
\hline Caída de Esfuerzos & 8.6217 & 34.7558 & \\
\hline Factor C & \multicolumn{2}{|c|}{4.0437} & \\
\hline Factor $\eta$ & \multicolumn{2}{|r|}{983} & \\
\hline Factor $\xi$ & \multicolumn{2}{|c|}{0.7221} & \\
\hline
\end{tabular}

Tabla 5.1. Datos de simulación 
La función de distribución de probabilidad (Fig. 5.3) será para este caso:

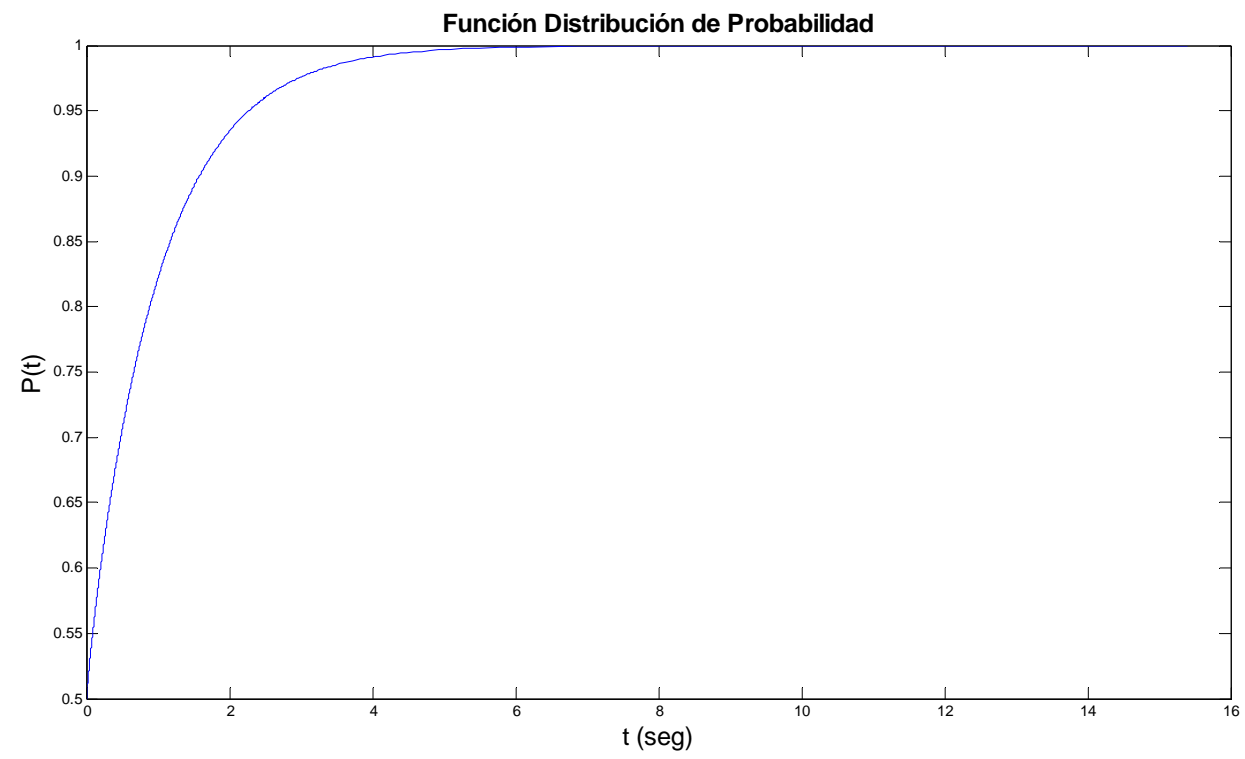

Figura 5.3 Función Distribución de Probabilidad para este caso. La rama para probabilidades de 0 a 0.5 es antisimétria a la que se muestra.

La función temporal equivalente a la fuente resultante de la simulación es (Fig. 5.4):

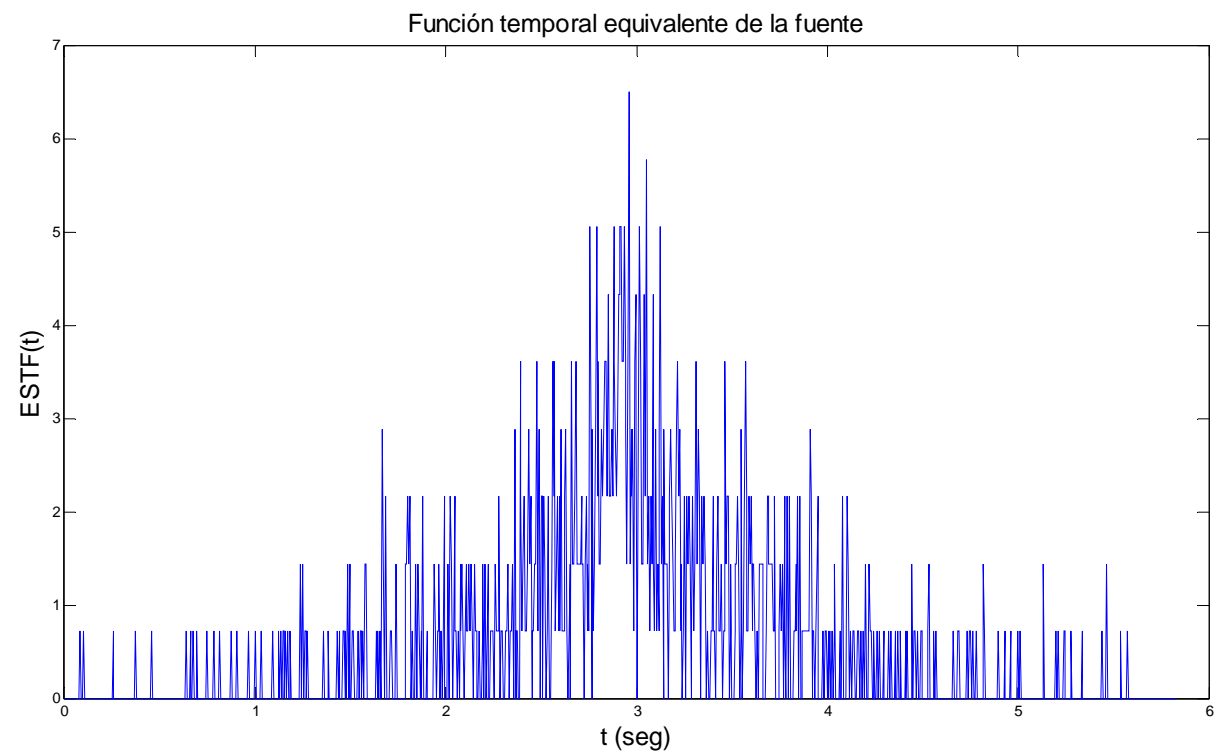

Figura 5.4. Función temporal equivalente a la fuente para esta simulación

y realizando la convolución entre esta función y los registros del evento función de Green empírica, obtenemos los registros simulados representados en la (Fig. 5.5) 

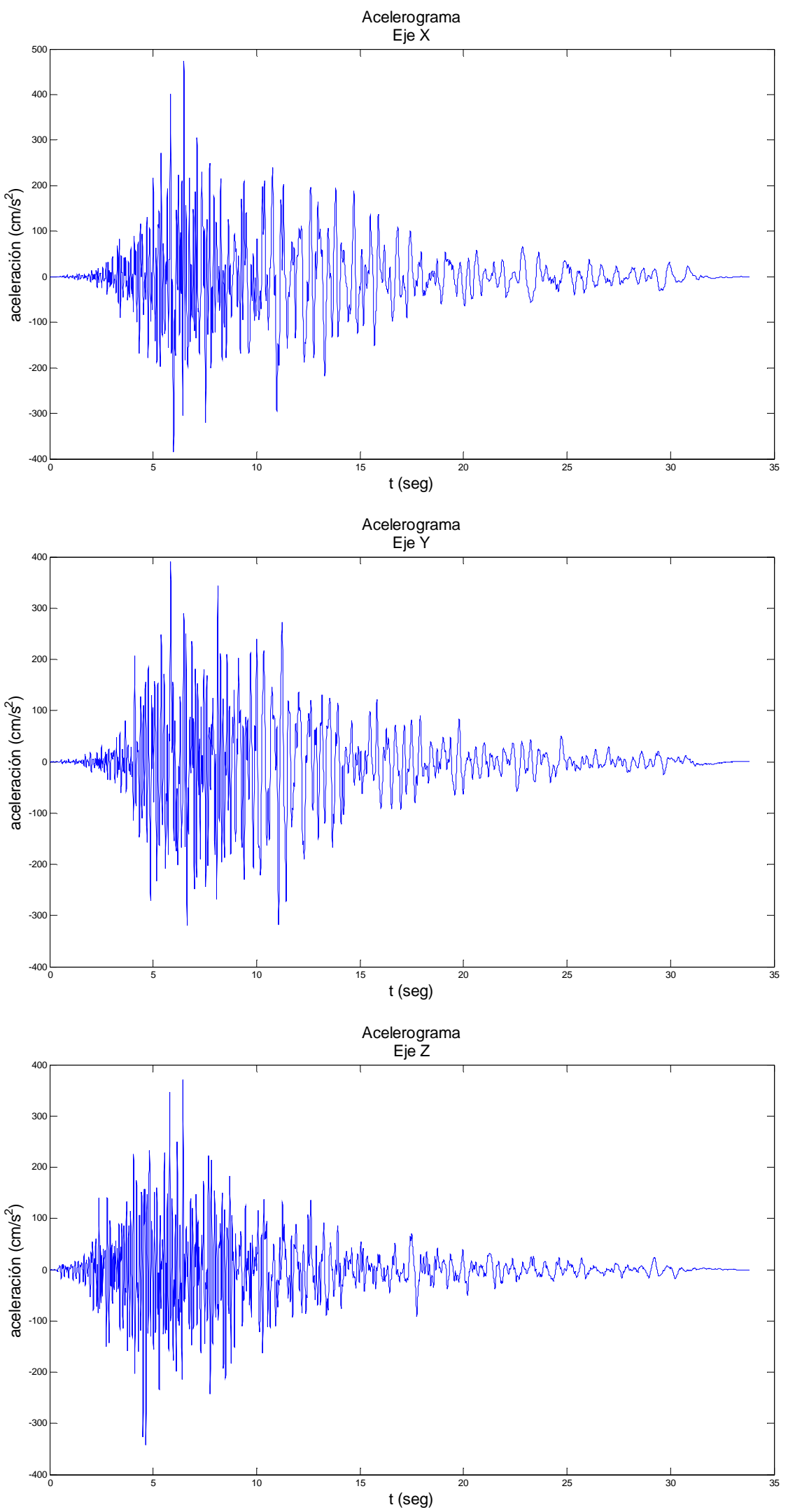

Figura 5.5. Acelerogramas del evento simulado 
Y los espectros elásticos de respuesta de aceleración son los de la Figura 5.6.
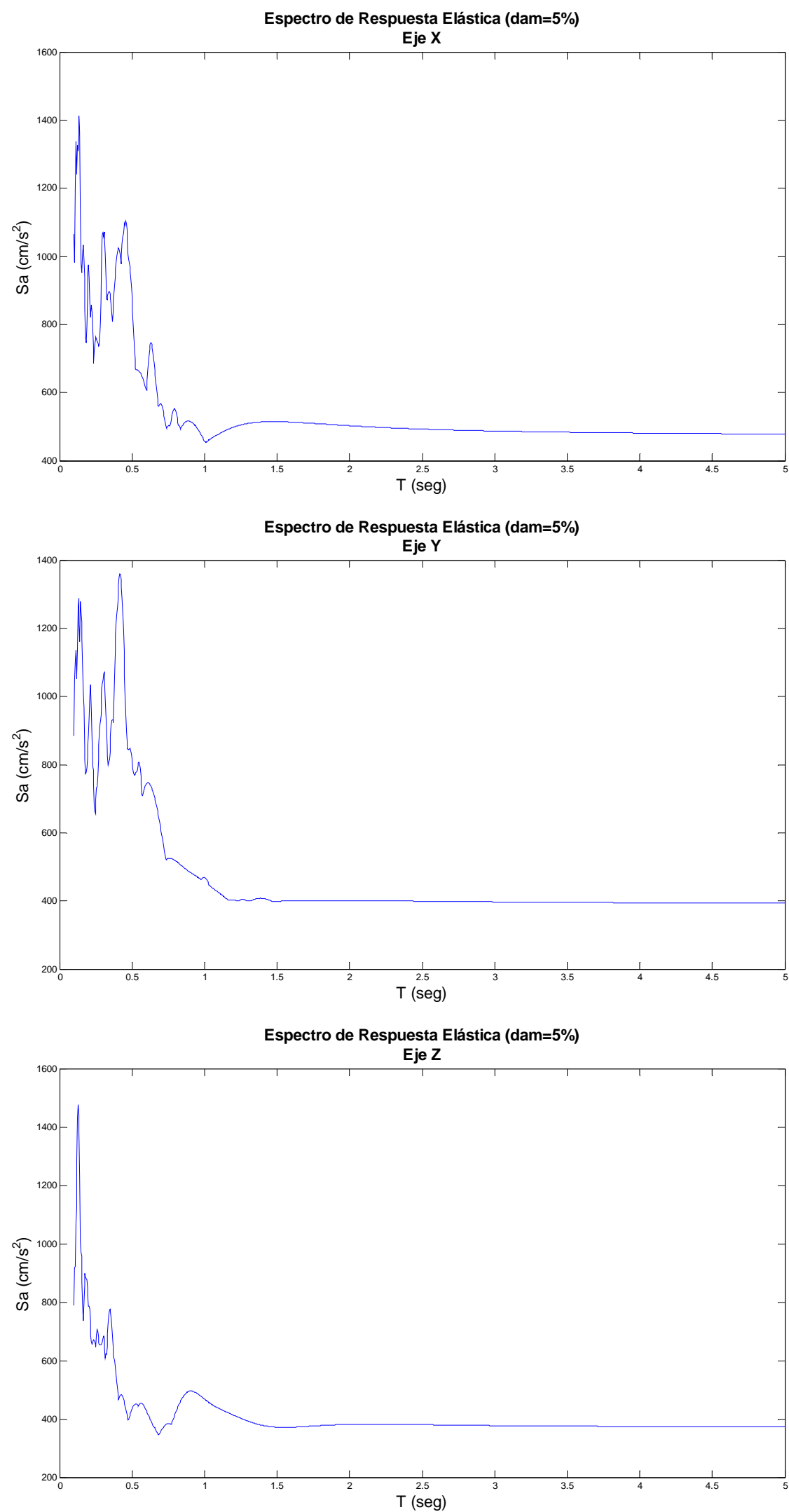

Figura 5.6. Espectros elásticos de respuesta de aceleración relativa para un amortiguamiento del $5 \%$ 
Como se puede observar, se trata de una simulación con un pico considerable en las altas frecuencias y una relación entre caídas de esfuerzos de un valor superior a 4, lo que nos hace pensar que es poco realista para los valores esperados en esta zona en cuestión, pero buen ejemplo de cómo se distribuirán los subeventos con la función temporal equivalente de la fuente.

En el siguiente ejemplo aumentamos el valor de la magnitud momento esperada y una frecuencia de esquina mucho menor, para así intentar que la relación entre caídas de esfuerzos se sitúe entre 1 y 2. Los valores de esta simulación son los de la Tabla 5.2.

Tabla 5.2. Datos de simulación

\begin{tabular}{|c|c|c|c|}
\hline Datos del Medio & & & \\
\hline Velocidad de Ondas S & \multicolumn{2}{|r|}{3.5} & \\
\hline Velocidad de Ruptura & \multicolumn{2}{|c|}{2.975} & \\
\hline Datos de los Eventos & Evento EGF & Evento Objetivo & \\
\hline Magnitud Momento & 4.1 & 6.9 & \\
\hline Momento Sísmico & $1.58 \mathrm{E}+15$ & $2.51 \mathrm{E}+19$ & \\
\hline Frecuencia de Esquina & 1.4 & 0.06 & \\
\hline Caída de Esfuerzos & 8.6217 & 10.7563 & \\
\hline Factor C & \multicolumn{2}{|c|}{1.2514} & \\
\hline Factor $\eta$ & \multicolumn{2}{|c|}{296420} & \\
\hline Factor $\xi$ & \multicolumn{2}{|c|}{0.0536} & \\
\hline
\end{tabular}

La función de distribución de probabilidad para este caso es la de la Figura 5.7.

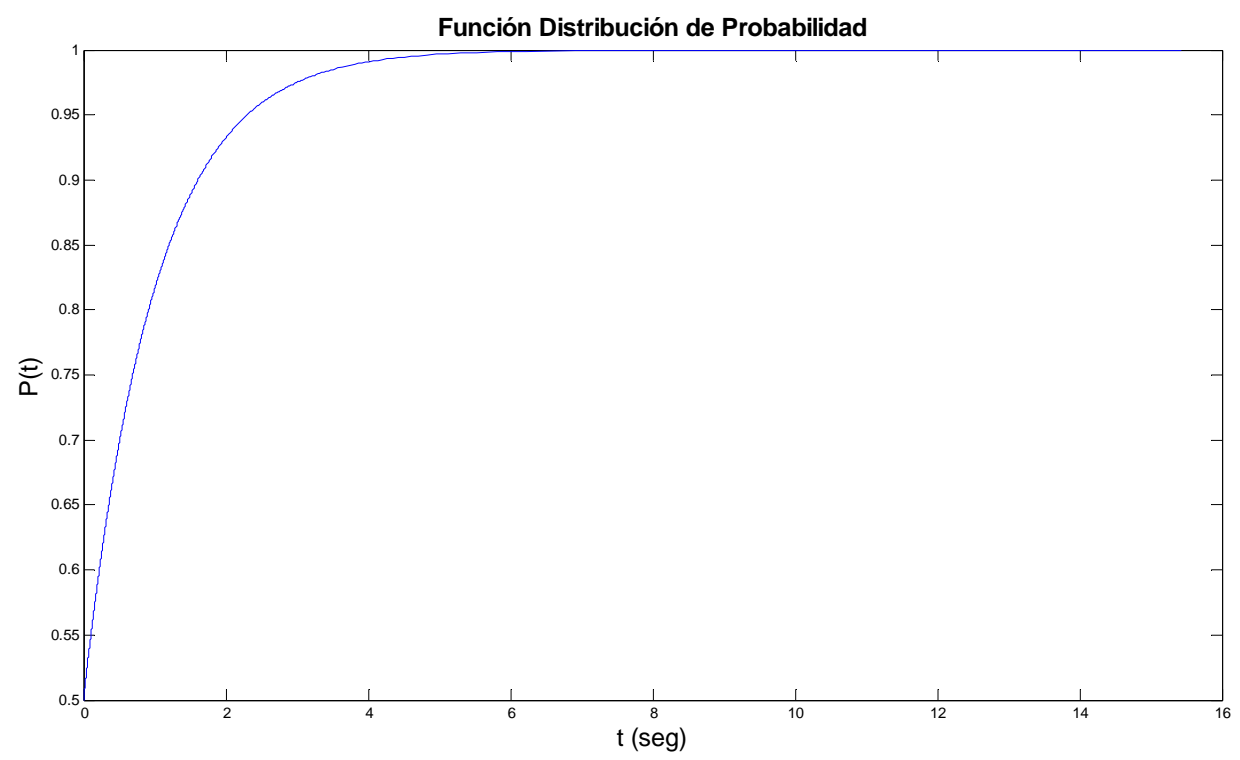

Figura 5.7 Función Distribución de Probabilidad para este caso. La rama para probabilidades de 0 a 0.5 es antisimétria a la que se muestra. 
La función temporal equivalente a la fuente resultante de la simulación es la de la Figura 5.8 .

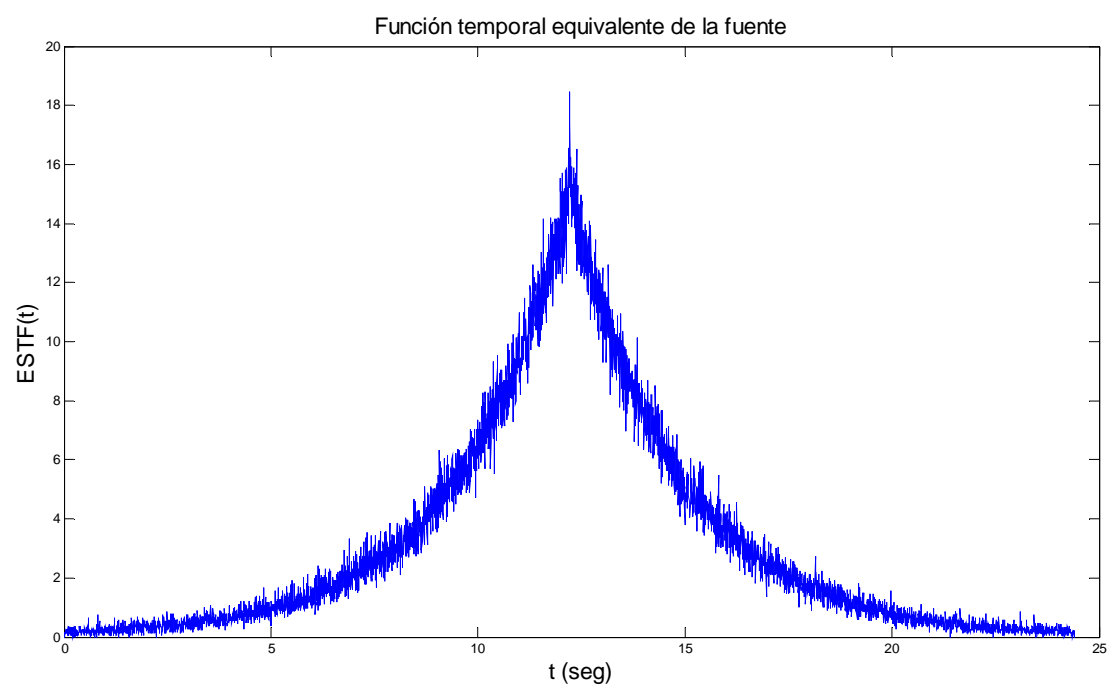

Figura 5.8. Función temporal equivalente a la fuente para esta simulación

y realizando la convolución entre esta función y los registros del evento función de Green empírica, obtenemos los registros simulados siguientes (Fig. 5.9). 

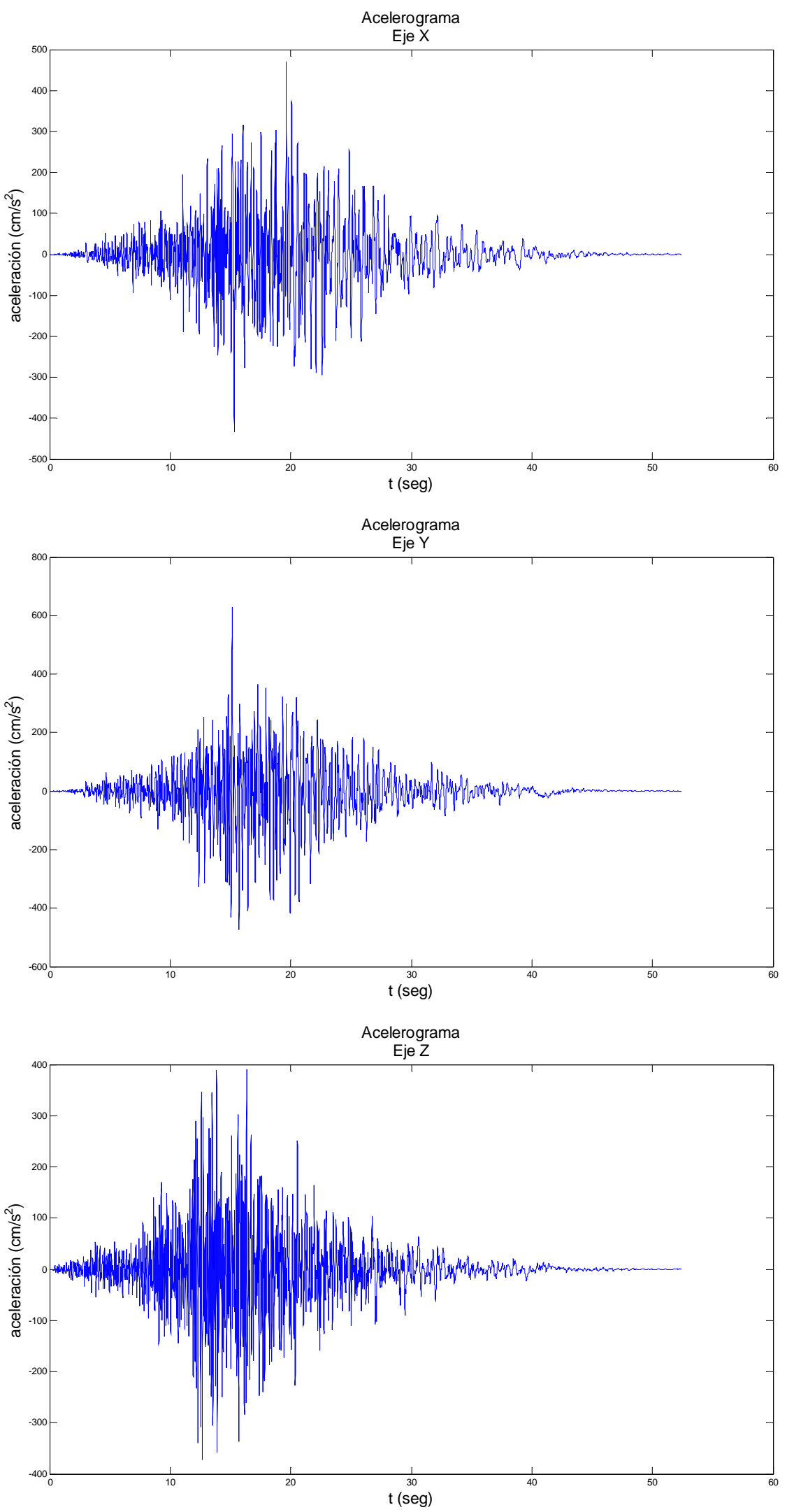

Figura 5.9. Acelerogramas del evento simulado 
Y los espectros elásticos respuesta de aceleraciones son los de la Figura 5.10.
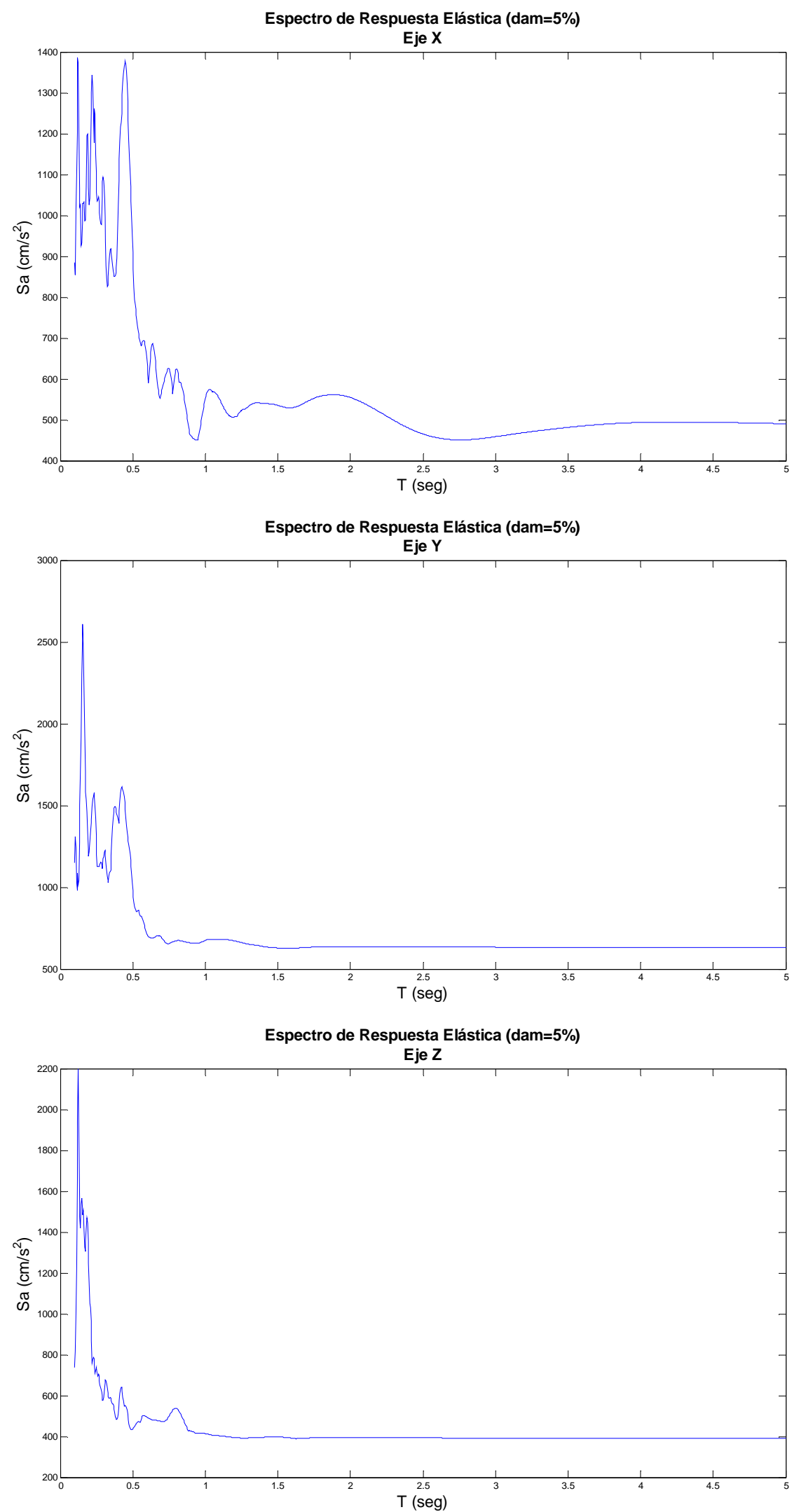

Figura 5.10. Espectros elásticos de respuesta de aceleración para un amortiguamiento del $5 \%$ 
$\mathrm{Al}$ igual que en la primera simulación, en las alta frecuencias se produce un pico bastante grande, lo cual nos puede llevar a pensar en que en esas frecuencias el registro EGF origen puede tener perturbaciones causadas por la instalación del acelerógrafo o del propio edificio donde se realiza la observación. Pero comparativamente, en esta segunda simulación se ha querido forzar algo más el modelado, no buscando resultados realistas, sino observar cómo se comporta al aumentar el valor de $\eta$ hasta valores sumamente altos, manteniendo una relación entre caída de esfuerzos, $C$, razonable del entorno de la unidad.

Recordemos que $\eta$ es el número de subeventos usados para realizar la simulación, los cuales son corregidos posteriormente por el factor $\xi$, que para este caso es muy pequeño. Es decir, se utilizan muchos subeventos, pero luego, al aplicarle el factor $\xi$, la contribución de cada uno es pequeña.

Este detalle será importante cuando se usen registros como función de Green empírica de mala calidad, o muy afectados por la instalación o el instrumento de medida. Al ser pequeña la contribución por cada subevento, estos errores acumulados serán menores, mientras que si las relaciones de escala hacen usar menos subeventos, la contribución de los errores de cada uno de estos, serán mucho mayores. 


\section{ENFOQUE SEMIEMPÍRICO. MODELADO CINEMÁTICO DE LA RUPTURA.}

Basándose en la idea de función de Green empírica de Hartzell (1978) y Kanamori (1979), Hadeley y Helemberger (1980) tomaron como plano de falla, para el evento a simular, un rectángulo mallado, divido en un número apropiado de planos pequeños de falla iguales al área del plano falla asociado con el evento más pequeño usado como función de Green empírica. Con esto se generaban un cierto número de eventos iguales al evento pequeño en cada subfalla, dentro de la duración esperada para el deslizamiento objetivo. No usaron una explicación clara del escalado entre los parámetros del evento objetivo y el evento origen, pero si relacionaron el área de falla y el rise time.

Madariaga e Irikura (1982) y Imagawa et al. (1984) desarrollaron métodos de simulación usando el concepto de función de Green empírica, introduciendo las relaciones de escala de los parámetros de la fuente, hecha por Kanamori y Anderson (1975).

Irikura (1983) consiguió una formulación compacta, combinando un modelo determinista de fuente cinemática con el escalado de los parámetros de la fuente, momento sísmico, longitud y anchura de la falla, deslizamiento final y rise time. Mostró que el movimiento intenso del terreno para un evento grande simulado por este método era coherente para un rango de frecuencias menor a la frecuencia correspondiente a la inversa del rise time del evento objetivo, pero que infravaloraba el rango de altas frecuencias superiores a esta frecuencia, cuando lo comparaba con el espectro del modelo $\omega^{2}$.

Irikura (1986) formula un método basado en la función de Green empírica para conseguir un escalado del espectro de la fuente tan bueno como pueda ser el escalado de los parámetros de la fuente, haciendo que tanto el evento objetivo como el evento menor o EGF (del inglés Empirical Green's Function) sigan un modelo $\omega^{2}$. La base física para esta formulación se describe en Aki e Irikura (1991), donde puede ser fácil extender el método a los casos donde el espectro de la fuente del evento objetivo se desvía del modelo $\omega^{2}$, usando un modelo de falla de barreras o de asperezas.

Boatwright (1988) desarrolla un método similar al de Irikura, usando filtros en el dominio de la frecuencia para lograr un escalado similar.

Una vez que Irikura propuso su método basado en un modelo determinista de fuente cinemática, muchos autores desarrollaron métodos basados en ello, como Takemura e Ikeura (1988), que introdujeron elementos estocásticos en el modelo de fuente determinista. Este método podía controlar la generación de altas frecuencias en el movimiento a partir de la parte estocástica a través de un parámetro que se regía por la 
desviación estándar de la distribución aleatoria de desplazamientos sobre el modelo de plano de falla.

Dan et al. (1989) desarrollan una fórmula que obedece el modelo $\omega^{2}$ de Brune usando un parámetro adicional para la caída de esfuerzos.

Aunque el problema fundamental está en las relaciones de escala y en la generación contenidos en altas frecuencias, aun aparecían ambigüedades sobre las características de la fuente, como la distribución de heterogeneidades en el plano de falla y la dependencia del tamaño de la fuente sobre la distribución de esfuerzos.

Más artículos publicados con aplicaciones, mejoras o testado del método de Irikura, son los de Midorikawa (1993) que lo usa para estimar aceleraciones pico esperadas; Irikura y Kamae (1994) y (1998) que pusieron en práctica el método para varios casos así como Wössner et al. (2002). Jarpe y Kasameyer (1996) realizaron una validación del método, Irikura, Kagawa y Sekiguchi (1997) hicieron una pequeña revisión sobre la función de velocidad deslizamiento; Kamae, Irikura y Pitarka (1998) desarrollan una técnica de simulación hibrida entre el método de la función de Green empírica y la función de Green simulada teóricamente para comprender el comportamiento de la ruptura en la fuente.

Pavic, Koller, et al. (2000) realizaron un estudio sobre las incertezas del método de Irikura, así como un estudio de los niveles confianza.

Pitarka, Somerville, Fukushima, et al. (2000) ampliaron el uso de la hibridación de funciones de Green para la simulación de los efectos en campo cercano de un evento sísmico, lo cual llevó a un desarrollo demasiado engorroso.

Importante para nuestro estudio es la publicación de Miyake, Iwata e Irikura (2003), en la que se caracteriza la fuente a través de un modelo cinemático heterogéneo para realizar las simulaciones de eventos sísmicos, la formulación de este artículo se explica con amplitud en apartados sucesivos. Este enfoque de las heterogeneidades se desarrolla para intentar lograr una técnica de la función de Green empírica, combinada con una teoría de ruptura dinámica sobre el plano de falla, comprendiendo el escalado y las complejidades de la fuente y que sea más manejable que formulaciones anteriores.

Con esta última metodología se han realizado numerosas publicaciones sobre simulaciones en zonas concretas, como Miyake et al. (2006), donde se intenta comprender el comportamiento de fuente para un terremoto histórico de 2005, o Poiata y Miyake (2006) para predecir futuros terremotos en Rumania, o en Bykova et al. (2010) donde se hace lo mismo para la zona de Moscú.

Después de explicar someramente la historia del arte de esta técnica, a la que podemos llamar "método de Irikura", nos centramos en explicar la formulación en los apartados sucesivos, así el cómo realizar la implementación del método. 


\subsection{Método de modelado cinemático basado en los postulados de Irikura.}

Comencemos describiendo la formulación a partir de Irikura (1986) para construir un movimiento intenso del terreno ligado a un terremoto grande, a partir del registro de un evento menor en la misma zona, ajustando los dos eventos al modelo de espectro fuente de Brune (modelo $\omega^{2}$ ), para luego introducir los cambios realizados en Miyake e Irikura et al. (2003) que será la formulación final que usaremos.

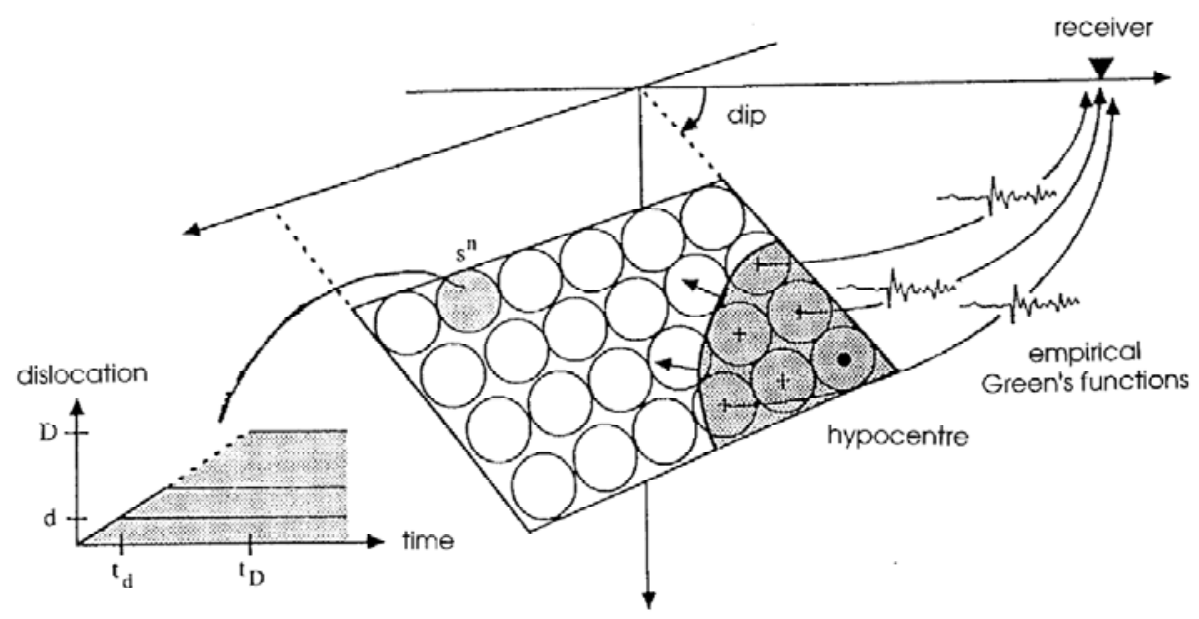

Figura 6.1. Síntesis del modelo cinemático basado en el uso de la función de Green Empírica

La idea fundamental del modelado cinemático de la fuente es la que se muestra en la imagen de la Figura 6.1. El plano de falla asociado al evento grande se supone compuesto por un número apropiado de fuentes del evento menor, las cuales rompen según una velocidad de deslizamiento fija o aleatoria, lo que provoca la llegada de los registros de del evento pequeño al receptor con unos tiempos de retardo, lo que hace que al superponerse, se obtenga un registro sintético de un evento mayor.

Este procedimiento se basa en las relaciones de escalado explicadas con anterioridad, que ahora volvemos a recordar para tenerlas presentes.

$$
\frac{L}{l}=\frac{W}{w}=\frac{D}{d}=\frac{T}{\tau}=N
$$

donde $L$ y $l$ son la longitud de la falla para un evento mayor y otro menor, $W$ y $w$ las anchuras de las respectivas fallas, $D$ y $d$ los desplazamientos finales, y $T$ y $\tau$ los respectivos rise time.

$$
\frac{\Delta \sigma_{o}}{\Delta \sigma_{s}}=C \quad \frac{M_{o o}}{M_{o s}}=C N^{3}
$$

donde $\Delta \sigma_{o}$ y $\Delta \sigma_{s}$ son las caídas de esfuerzos del evento objetivo y del evento pequeño respectivamente, y $M_{O o}$ y $M_{O S}$ los momentos sísmicos de los dos eventos. De estas relaciones, operando se puede obtener que

$$
N=\frac{f_{c S}}{f_{c o}} \quad C=\frac{M_{o o}}{M_{o s}} \cdot N^{-3}
$$


Irikura, en la publicación de 1986 y posteriores, toma con valor unidad la relación entre las caídas de esfuerzos, como si fueran independientes del momento sísmico cada uno de los eventos y fuese igual para los dos casos. Para nuestro caso dejaremos que la relación pueda ser distinta de la unidad.

Irikura divide el plano de falla en $N x N$ elementos, tal y como se puede observar en el siguiente esquema de los parámetros (Fig. 6.2.)

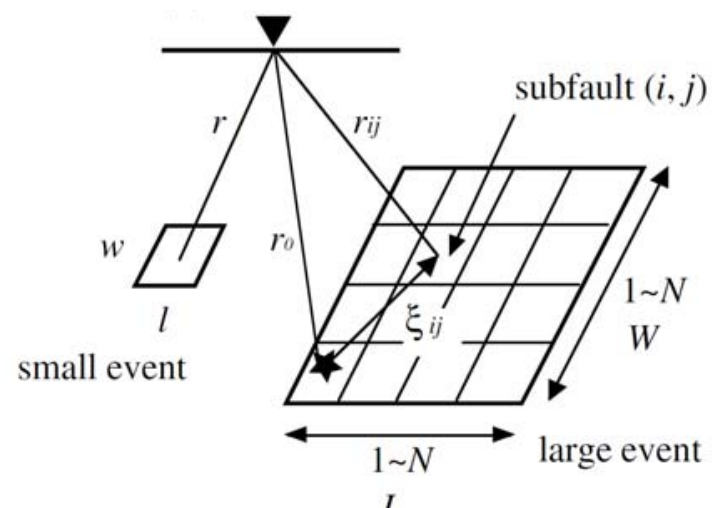

Figura 6.2. Relaciones de escala entre el evento objetivo y el evento EGF

Además de los relacionados con el escalado, en la Fig. 6.2. aparecen otros parámetros no explicados aún. $r$ es la distancia entre el hipocentro del evento menor y el punto de observación, $r_{o}$ es la distancia entre el hipocentro del evento objetivo y el punto de observación, $r_{i j}$ la distancia desde el punto de observación a cada uno de los hipocentros de los subeventos que componen el evento mayor y la matriz $\xi_{i j}$ la llamaremos matriz de distancias, y para cada elemento $(i, j)$ del plano de falla, $\xi_{i j}$ nos da la distancia al hipocentro del evento simulado.

De aquí en adelante vamos a suponer que este hipocentro del evento simulado está situado en la parte inferior del plano de falla. Esto lo hacemos por simpleza a la hora de expresarnos, ya que como el comienzo de la ruptura en el plano lo determinamos deterministicamente, solo habría que situarlo en otro punto cualquiera del plano y cambiar la matriz de distancias para que se acomodase a ese modelo.

Una vez determinados los parámetros a usar, podemos plantear la formulación del método, a partir de la expresión general de los métodos semiempíricos:

$$
S(t)=\operatorname{ESTF}(t) * S(t)
$$

es decir, que el registro sintético para un evento sísmico mayor registrado en un determinado punto de observación, $S(t)$, se obtiene de la convolución de la función temporal equivalente de la fuente, $\operatorname{ESTF}(t)$, con el registro del evento más pequeño registrado en ese mismo punto de observación y con hipocentro en la misma zona que el supuesto evento mayor. 
La función temporal equivalente de la fuente tiene la siguiente forma

$$
\operatorname{ESTF}(t)=\kappa \cdot \sum \delta\left(t-t_{i}\right)
$$

siendo para el método de Irikura esta función igual a

$$
\operatorname{ESTF}(t)=C \cdot \sum_{i=1}^{N} \sum_{j=1}^{N} \frac{r}{r_{i j}} F_{i j}(t)
$$

es decir, que el registro sintético del evento objetivo vendrá dado por

$$
S(t)=\left(C \cdot \sum_{i=1}^{N} \sum_{j=1}^{N} \frac{r}{r_{i j}} F_{i j}(t)\right) * s(t)
$$

donde $F_{i j}$ es la función de filtrado.

Irikura (1986) y Miyake e Irikura et al. (2003) dan dos expresiones similares para la función de filtrado, aunque en Irikura (1986) se sigue usando un solo índice numerador para determinar los subeventos, arrastrando un concepto de linealidad en vez de representarlo como un subespacio bidimensional.

La función de filtrado en Irikura (1986) es

$$
F_{i}(t)=\delta\left(t-t_{i}\right)+\frac{1}{n^{\prime}} \sum_{k=1}^{(N-1) n^{\prime \prime}}\left[\delta\left(t-t_{i}-\frac{(k-1) T}{(N-1) n^{\prime}}\right)\right]
$$

con $i=1,2, \ldots N x N$, y $n^{\prime}$ un valor entero apropiado para eliminar posibles periodicidades falsas, este valor se toma según los autores entre 20 y 30 , y donde $t_{i}$ es el tiempo de retardo en cada subevento, expresado por

$$
t_{i}=\frac{r_{i}-r_{o}}{V_{s}}+\frac{\xi_{i}}{V_{r}}+\epsilon_{i}
$$

siendo $V_{S}$ la velocidad de las ondas $\mathrm{S}, V_{r}$ la velocidad de ruptura, y $\epsilon_{i}$ es la parte aleatoria de los tiempos de retardo, que utiliza el parámetro $c$ que varía aleatoriamente entre -1 y 1, y con eso tenemos que

$$
\epsilon_{i}=\frac{c \cdot \Delta w}{2 \cdot V_{r}}
$$

es decir que si nos fijamos bien, lo que se está es modificando la componente de la matriz de distancias, emulando un aumento o decremento de la cantidad, para así conseguir que lo que realmente varíe sea la velocidad de ruptura. De hecho, en la formulación siguiente 
de Miyake e Irikura et al. (2003), no aparece un término equivalente a $\epsilon_{i}$ en la expresión de los tiempos de retardo, simplemente se dice que la variación aleatoria se consigue haciendo aleatoria la velocidad de ruptura, pero no fija esa aleatoriedad de alguna manera.

La formulación en Miyake e Irikura et al. (2003) para la función de filtrado es la siguiente

$$
\begin{gathered}
F_{i j}(t)=\delta\left(t-t_{i j}\right)+\frac{1}{n^{\prime} \cdot\left(1-\frac{1}{e}\right)} \sum_{k=1}^{(N-1) n^{\prime}}\left[\frac{1}{\left.e^{\frac{k-1}{(N-1) n^{\prime}}} \delta\left(t-t_{i j}-\frac{(k-1) T}{(N-1) n^{\prime}}\right)\right]}\right. \\
t_{i j}=\frac{r_{i j}-r_{o}}{V_{s}}+\frac{\xi_{i j}}{V_{r}}
\end{gathered}
$$

muy parecida a la formulación de Irikura (1886), aunque con algunos cambios como el que cuente ya con indicadores de la posición de los subeventos en el plano de falla con los elemento $(i, j)$.

La nueva función de filtrado tiene un decaimiento exponencial en vez de una función en forma de caja como se mantuvo hasta Irikura et al. (1997). El que por cada subevento en la función de filtrado aparezca, además del valor impulsivo unitario, ese decaimiento exponencial o esa especie de peine de $(N-1) n^{\prime}$ pequeños valores impulsivos, es por lo que se habló en el enfoque teórico de las paradas y arranques, que son los mecanismos con los que se consiguen los contenidos en altas frecuencias. En la imagen siguiente (Fig. 6.3), se pueden ver las diferencias entre un tipo de función de filtrado y otro.
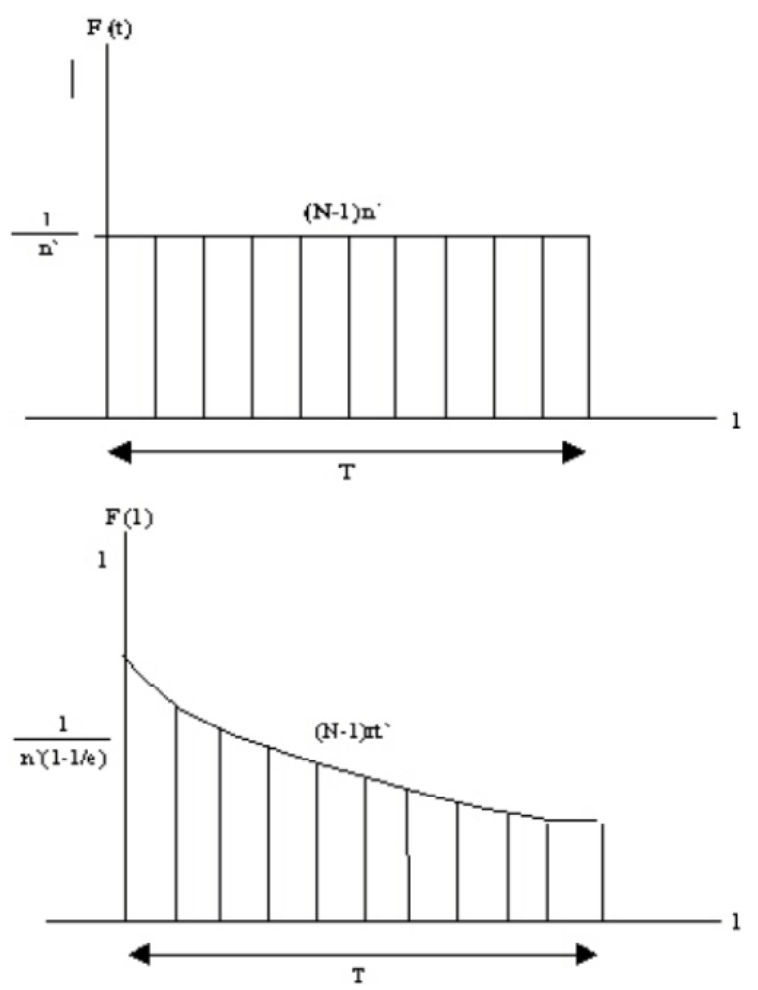

Figura 6.3. Función de filtrado de Irikura (1986) y de Miyake e Irikura (2003) 
El significado físico de la formulación se basa en tomar el evento pequeño y los subeventos que forman el evento mayor como grietas circulares como las que Madariaga (1976) estudió dinámicamente. Y como se ha dicho antes, para que estas roturas generen movimientos que espectralmente correspondan con el modelo $\omega^{2}$, debe de haber paradas y arranques para poder asemejar los contenidos espectrales en las altas y bajas frecuencias al escalado $\omega^{2}$.

Este método de uso de la función de Green empírica tiene la ventaja no solo de contener los efectos del camino y del sitio sino que también incorpora los efectos de la fuente debidos al crecimiento de pequeñas roturas dentro de la rotura mayor.

\subsection{Implementación del método de modelado cinemático.}

En el apartado anterior nos hemos centrado en lo que es lo formulación desarrollada a partir del método de Irikura, para conseguir simular eventos grandes a partir de otro pequeños, usando estos a modo de función de Green empírica. En este apartado vamos a intentar explicar la manera de implementar este método, con la ayuda de cualquier programa que sea capaz de programar cálculos numéricos. Empezaremos fijando los datos de entrada necesarios y los objetivos a realizar, es decir los datos de salida esperados, y luego explicaremos las particularidades de la implementación.

\subsubsection{Datos generales del medio.}

El medio solo tenemos que caracterizarlo por:

- Velocidad de las ondas $S, V_{S}$.

- Velocidad de ruptura, $V_{r}$, que se puede suponer entre un 80 o un $90 \%$ del valor de las ondas S.

\subsubsection{Datos de la función de Green empírica.}

Los datos principales para definir la función de Green empírica son:

- Registros en las tres direcciones del espacio. Normalmente acelerogramas o sismogramas con una determinada frecuencia de muestreo, la cual también será para las simulaciones.

- Coordenadas terrestres del hipocentro y de la estación donde se registra. Con estas coordenadas obtener la distancia es tan sencillo como calcular la distancia entre dos puntos en el espacio. 
Además, se tomará como origen de coordenadas del sistema vectorial necesario, para calcular distancias y vectores asociados, el centrado en el punto de la estación de observación.

- Magnitud momento y frecuencia de esquina.

De la magnitud momento despejando de la expresión de Kanamori

$$
M_{w}=\frac{2}{3} \log _{10} M_{o}-10.7
$$

obtenemos el momento sísmico asociado al evento EGF.

Conociendo el momento sísmico y la frecuencia de esquina, podemos determinar la caída de esfuerzos, si el espectro de desplazamientos cumple el modelo de Brune, a través de la expresión

$$
\Delta \sigma=M_{o}\left(\frac{f_{c}}{4.9 \cdot 10^{6} \cdot \beta}\right)^{3}
$$

donde $\beta$ es la velocidad de las ondas S expresada en $\mathrm{km} / \mathrm{s}, \Delta \sigma$ está en bar y $M_{o}$, momento sísmico, en dina-cm.

- Mecanismo focal del evento. De los datos del mecanismo focal precisamos el acimut, $\phi$, y el buzamiento, $\delta$, con los cuales poder generar un plano geométrico que tenga por direcciones principales estos y que se apoye en el punto del hipocentro.

El vector unitario que marca la dirección del acimut viene dado por

$$
\overrightarrow{v_{s}}=(\sin (\phi), \cos (\phi), 0)
$$

y el vector unitario que marca la dirección del buzamiento por

$$
\overrightarrow{v_{d}}=(-\cos (\phi) \cos (\delta), \sin (\phi) \cos (\delta), \sin (\delta))
$$

- Longitud y anchura de la falla del evento pequeño, que vendrán dadas por las expresiones

$$
\log _{10} l=\frac{1}{2}\left(\log _{10} M_{o s}-23.58\right)
$$

donde $L$ se expresa en $\mathrm{km}$, y $M_{o}$ en dina-cm, y

$$
w=\frac{l}{2}
$$


- $\quad$ Rise time, que viene dado por la expresión de Savage (1972)

$$
\tau_{r s}=\frac{w}{2 V_{r}}=\frac{l}{4 V_{r}}
$$

\subsubsection{Datos del evento objetivo.}

Para el evento objetivo a calcular se fijan los siguientes datos

- Magnitud momento, frecuencia de esquina y caída de esfuerzos, de la misma manera que se han calculado para el evento menor o evento función de Green empírica.

- Se calculan los parámetros de escaldo $C$ y $N$

$$
\frac{\Delta \sigma_{o}}{\Delta \sigma_{s}}=C \quad N=\frac{f_{c S}}{f_{c o}}
$$

- Se calculan los parámetros de la falla longitud, anchura, y rise time a partir de la relación siguiente

$$
\frac{L}{l}=\frac{W}{w}=\frac{T}{\tau}=N
$$

- El mecanismo focal va a ser el mismo para los dos eventos. Solo hará falta fijar determinativamente un nuevo punto dentro del plano geométrico donde está contenido el plano de falla como hipocentro del evento objetivo. Con este punto fijado, se tiene la distancia del hipocentro del evento objetivo a la estación.

La expresión para obtener este punto es

$$
\overrightarrow{v_{r o}}=\overrightarrow{v_{r}}+a \overrightarrow{v_{s}}+b \overrightarrow{v_{d}}
$$

siendo $\overrightarrow{v_{r}}$ el vector de posición del hipocentro del evento EGF con respecto al origen de coordenadas situado en la estación registro.

\subsubsection{Función de Filtrado.}

La función de filtrado ya explicada

$$
\begin{gathered}
F_{i j}(t)=\delta\left(t-t_{i j}\right)+\frac{1}{n^{\prime} \cdot\left(1-\frac{1}{e}\right)} \sum_{k=1}^{(N-1) n^{\prime}}\left[\frac{1}{\left.e^{\frac{k-1}{(N-1) n^{\prime}}} \delta\left(t-t_{i j}-\frac{(k-1) T}{(N-1) n^{\prime}}\right)\right]}\right. \\
t_{i j}=\frac{r_{i j}-r_{o}}{V_{s}}+\frac{\xi_{i j}}{V_{r}}
\end{gathered}
$$


de la que para poder implementarla es necesario calcular los siguientes datos;

- Matriz de distancias de subeventos a la estación de observación, que se realiza calculando el módulo de los vectores de posición de cada subevento, que están contenidos dentro del plano de falla.

$$
\overrightarrow{v_{r l j}}=\overrightarrow{v_{r o}}+i \cdot l \cdot \overrightarrow{v_{s}}+j \cdot w \cdot \overrightarrow{v_{d}}
$$

como se puede ver, gracias a que usamos vectores unitarios para parametrizar el plano, se puede claramente obtener las posiciones a través de los indicadores de posición $(i, j)$ dentro del plano de falla del evento objetivo, multiplicados por la distancia de cada celda del mallado, es decir la longitud y la anchura del evento origen.

- $\quad \xi_{i j}$ seria la matriz de distancias dentro del plano de falla del evento objetivo. Se calcula conociendo la distancia del centro de cada celda del mayado con la que es hipocentro del evento.

En el código desarrollado, se ha tomado como origen fijo el extremo inferior izquierdo del mayado, para otro tipo de ruptura, se puede implementar fácilmente la matriz de distancias correspondiente.

- Para obtener la matriz de tiempos de retardo $t_{i j}$, es necesario conocer cómo va a variar aleatoriamente la velocidad de ruptura, por lo cual generamos otra matriz de velocidades aleatorias de ruptura, la cual tendremos que desarrollar igualando las dos formulaciones de $t_{i j}$, la de Irikura (1986) y la de Miyake e Irikura et al. (2003). Estas son respectivamente

$$
t_{i}=\frac{r_{i}-r_{o}}{V_{s}}+\frac{\xi_{i}}{V_{r}}+\epsilon_{i}
$$

siendo $V_{S}$ la velocidad de las ondas $S, V_{r}$ la velocidad de ruptura, y $\epsilon_{i}$ es la parte aleatoria de los tiempos de retardo, que utiliza el parámetro $c$ que varía aleatoriamente entre -1 y 1 , y con eso tenemos que

$$
\epsilon_{i}=\frac{c \cdot \Delta w}{2 \cdot V_{r}} \quad \text { y } \quad t_{i j}=\frac{r_{i j}-r_{o}}{V_{s}}+\frac{\xi_{i j}}{V_{r}}
$$

Como sabemos que

$$
\frac{\xi_{i}}{V_{r}}+\frac{c \cdot \Delta w}{2 \cdot V_{r}}=\frac{\xi_{i j}}{V_{\text {rale }}}
$$

donde $V_{\text {rale }}$ es la velocidad de ruptura aleatoria, y podemos despejar obteniendo:

$$
V_{\text {rale }}=V_{r} \cdot\left(\frac{2 \xi_{i j}}{2 \xi_{i j}+c \cdot \Delta w}\right)
$$


$\Delta w$ es simplemente el valor de la anchura del evento origen. Este artificio se realiza para conseguir velocidades de ruptura dentro de unos límites, así nos aseguramos que no se obtengan tiempos de ruptura demasiado largos.

El promedio de las velocidades de ruptura, será la velocidad de ruptura estimada entre el 80 y el $90 \%$ de la velocidad de las ondas S.

- Para el cálculo de la función de filtrado se recomienda el uso de matrices cubicas de dimensiones NxNxnt, donde $n t$ es el número de datos temporales que ocuparían toda la función de filtrado.

\subsubsection{Obtención de la función temporal equivalente de la fuente.}

La función ESTF se consigue simplemente con la aplicación de la expresión

$$
\operatorname{ESTF}(t)=C \cdot \sum_{i=1}^{N} \sum_{j=1}^{N} \frac{r}{r_{i j}} F_{i j}(t)
$$

Con lo que aplicando la convolución ya tenemos una simulación por el método de Irikura, según los parámetros que buscábamos como objetivos.

$$
S(t)=\left(C \cdot \sum_{i=1}^{N} \sum_{j=1}^{N} \frac{r}{r_{i j}} F_{i j}(t)\right) * S(t)
$$

\subsection{Simulaciones con el método de Irikura.}

La formulación está más que probada por los diversos autores que han trabajado sobre ella. Apliquemos el método a terremotos de nuestro entorno.

Usamos el terremoto del 24 de Febrero de 1997 de Agrón, que se puede ver en el Anejo 1, para realizar dos simulaciones del modelo cinemático para comparar las salidas de una y otra. El registro de este evento fue el del Observatorio de Cartuja, en Granada, en un terreno clasificado como roca.

La primera simulación será con una magnitud momento esperada 6.3 y una frecuencia de esquina esperada de $0.2 \mathrm{~Hz}$. Los datos de entrada y de salida para esta simulación se recogen en la Tabla 6.1 . 
Tabla 6.1. Datos de la simulación

\begin{tabular}{|c|c|c|c|}
\hline Datos del Medio & & & \\
\hline Velocidad de Ondas S & \multicolumn{2}{|r|}{3.5} & \\
\hline Velocidad de Ruptura & \multicolumn{2}{|c|}{2.975} & \\
\hline Datos de los Eventos & Evento EGF & Evento Objetivo & \\
\hline Magnitud Momento & 4.3 & 6.3 & \\
\hline Momento Sísmico & $2.94 \mathrm{E}+15$ & $3.16 \mathrm{E}+18$ & \\
\hline Frecuencia de Esquina & 1.2 & 0.2 & \\
\hline Caída de Esfuerzos & 11 & 50.1531 & \\
\hline Factor N & \multicolumn{2}{|r|}{6} & \\
\hline Factor C & \multicolumn{2}{|c|}{4.9797} & \\
\hline
\end{tabular}

La función temporal equivalente de la fuente resultante de la simulación se muestra a continuación en la Fig. 6.4.

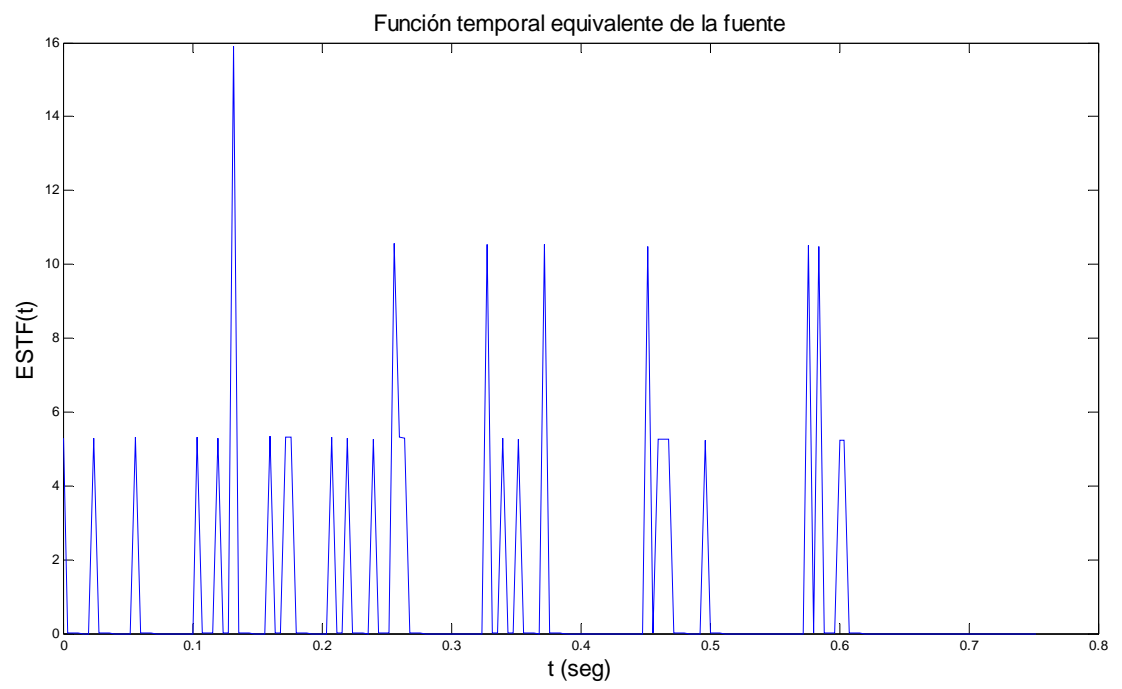

Figura 6.4. Función temporal equivalente de la fuente para este caso concreto

y realizando la convolución entre esta función y los registros del evento función de Green empírica, obtenemos los registros simulados, representados en la Figura 6.5. 

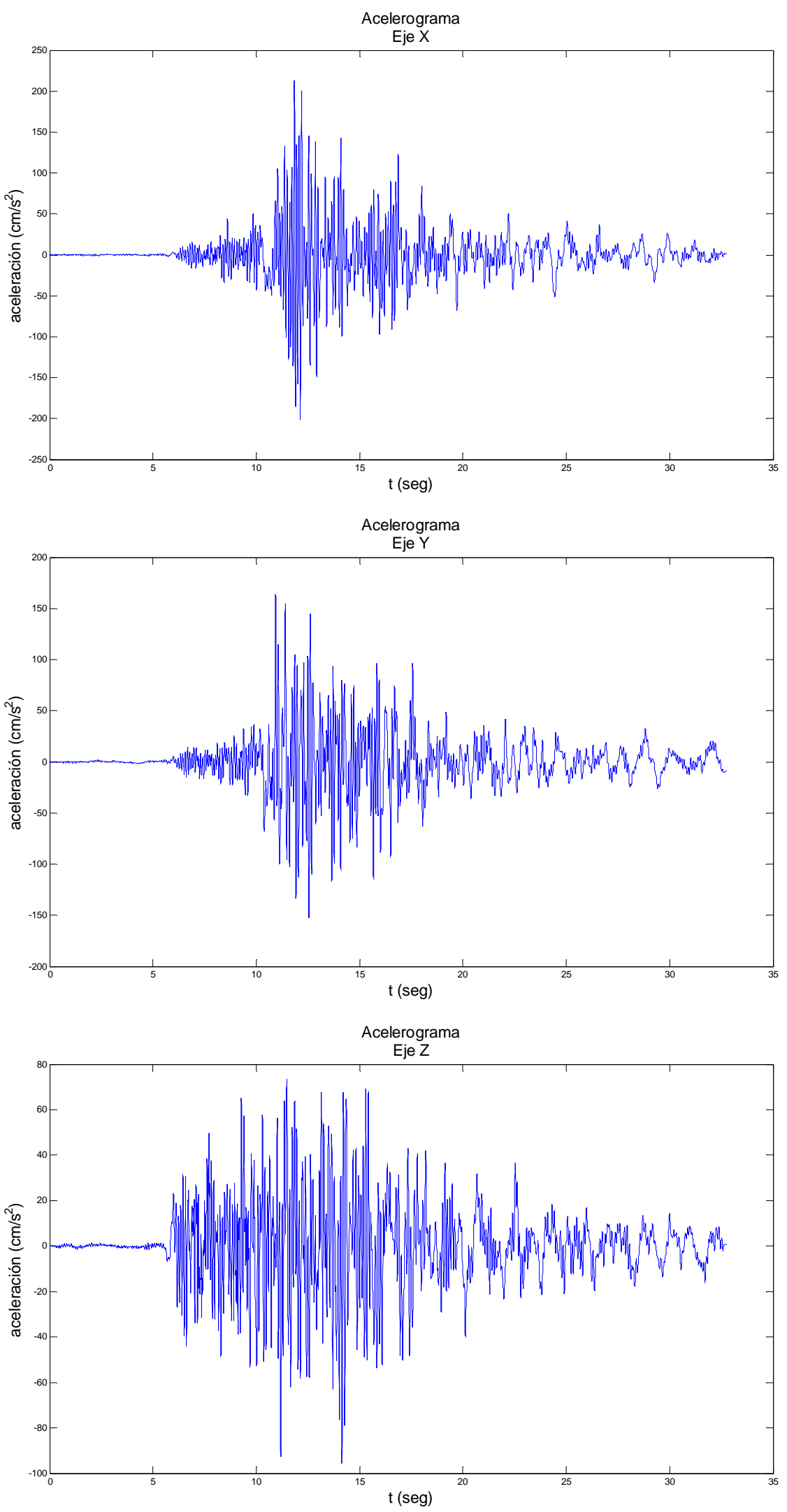

Figura 6.5. Acelerogramas del evento simulado 
Los espectros elásticos de respuesta para un amortiguamiento del 5\% son ( Fig. 6.6.):
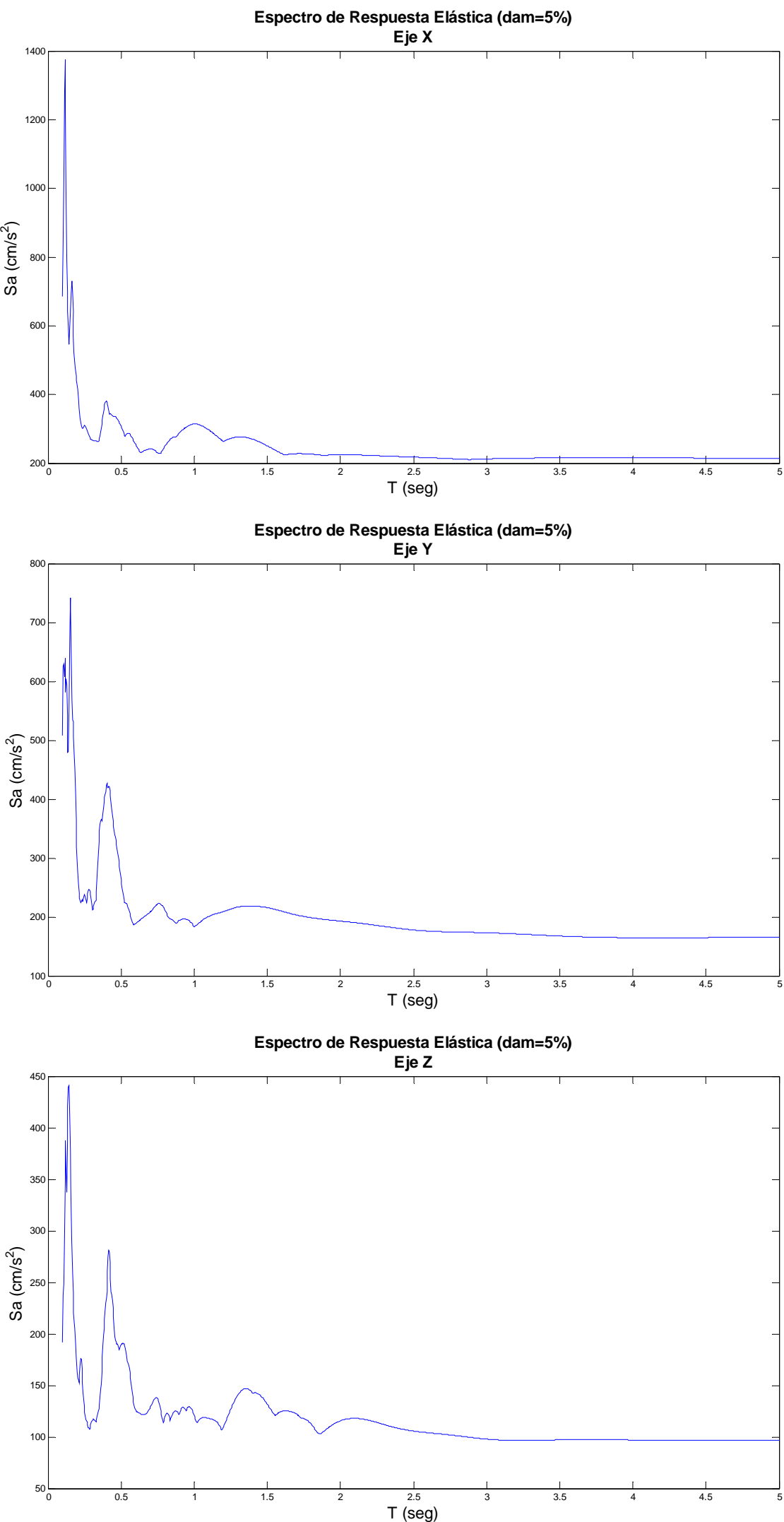

Figura 6.6. Espectros elásticos de respuesta de aceleración para una amortiguamiento del $5 \%$ 
Como se puede observar se producen aceleraciones muy importantes sobre todo respecto al eje $x$, donde se aprecia un pico en el espectro muy importante. Téngase en cuenta que la relación $C$, entre las caídas de esfuerzos tiene un valor cercano a 5, si se acercase a 1 los valores de los espectros de aceleración serían menores.

Aumentando más la magnitud momento esperada, pero haciendo que $C$ sea más bajo, realizamos una segunda simulación con los siguientes datos (Tabla 6.2.):

Tabla 6.2. Datos de la simulación

\begin{tabular}{|c|c|c|c|}
\hline Datos del Medio & & & \\
\hline Velocidad de Ondas S & \multicolumn{2}{|r|}{3.5} & \\
\hline Velocidad de Ruptura & \multicolumn{2}{|c|}{2.975} & \\
\hline Datos de los Eventos & Evento EGF & Evento Objetivo & \\
\hline Magnitud Momento & 4.3 & 6.9 & \\
\hline Momento Sísmico & $2.94 \mathrm{E}+15$ & $2.51 \mathrm{E}+19$ & \\
\hline Frecuencia de Esquina & 1.2 & 0.07 & \\
\hline Caída de Esfuerzos & 11 & 17.0805 & \\
\hline Factor N & \multicolumn{2}{|r|}{18} & \\
\hline Factor C & \multicolumn{2}{|c|}{1.6959} & \\
\hline
\end{tabular}

La función temporal equivalente de la fuente resultante en esta simulación es la que se muestra continuación en la Fig. 6.7.

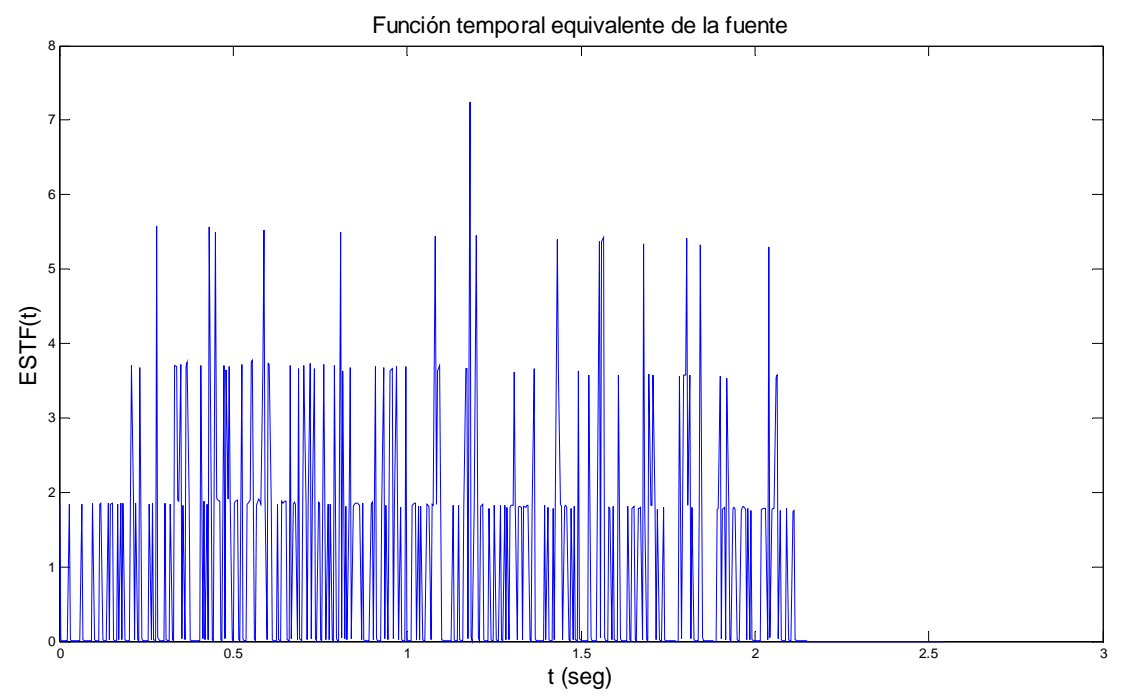

Figura 6.7. Función temporal equivalente de la fuente 
Realizando la convolución entre esta función y los registros del evento función de Green empírica, obtenemos los registros simulados que se muestran en la Fig. 6.8. y los respectivos espectros de respuesta de aceleración para un amortiguamiento del 5\%, (Fig. 6.9). 

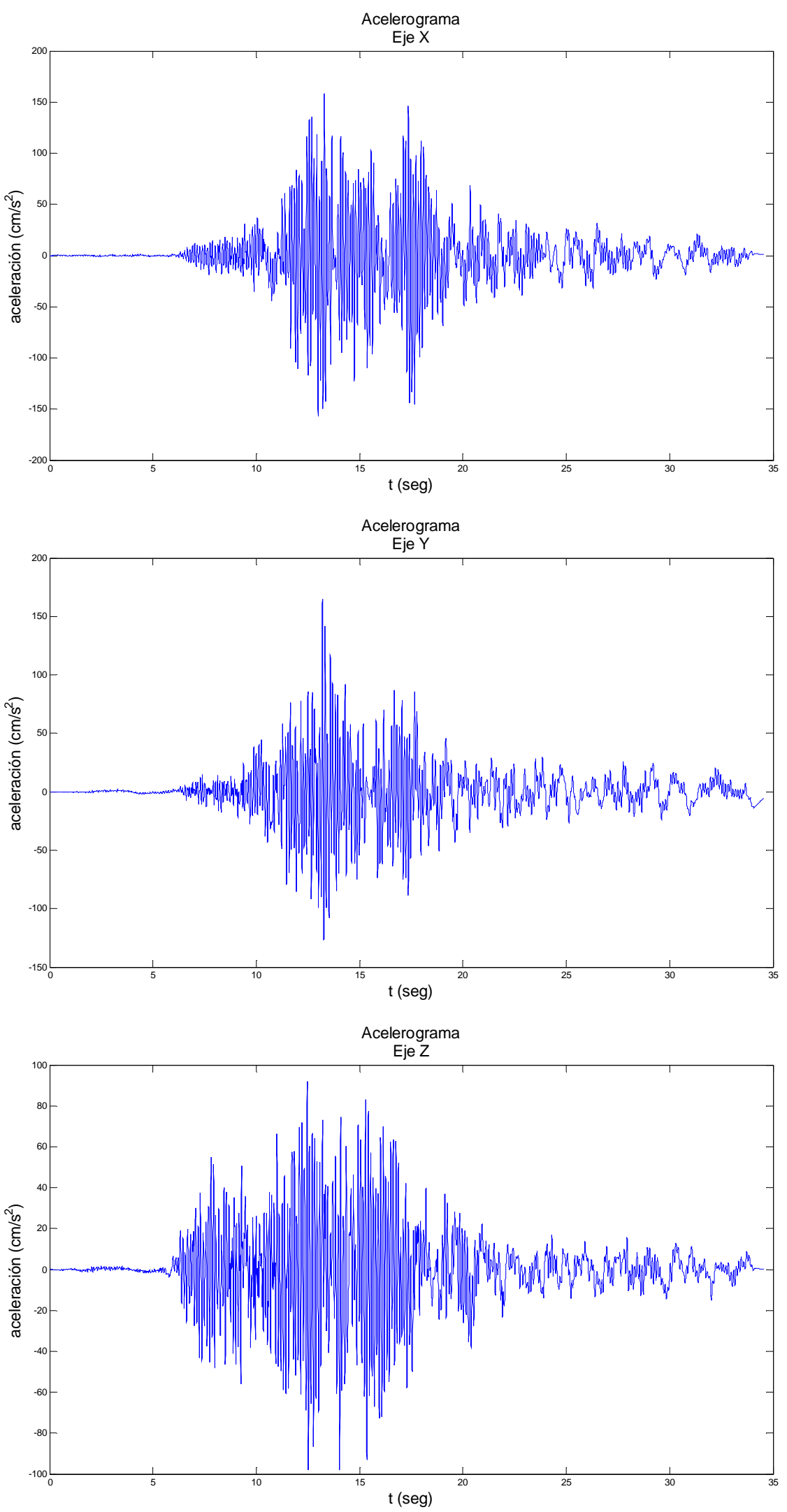

Figura 6.8. Acelerogramas del evento simulado 

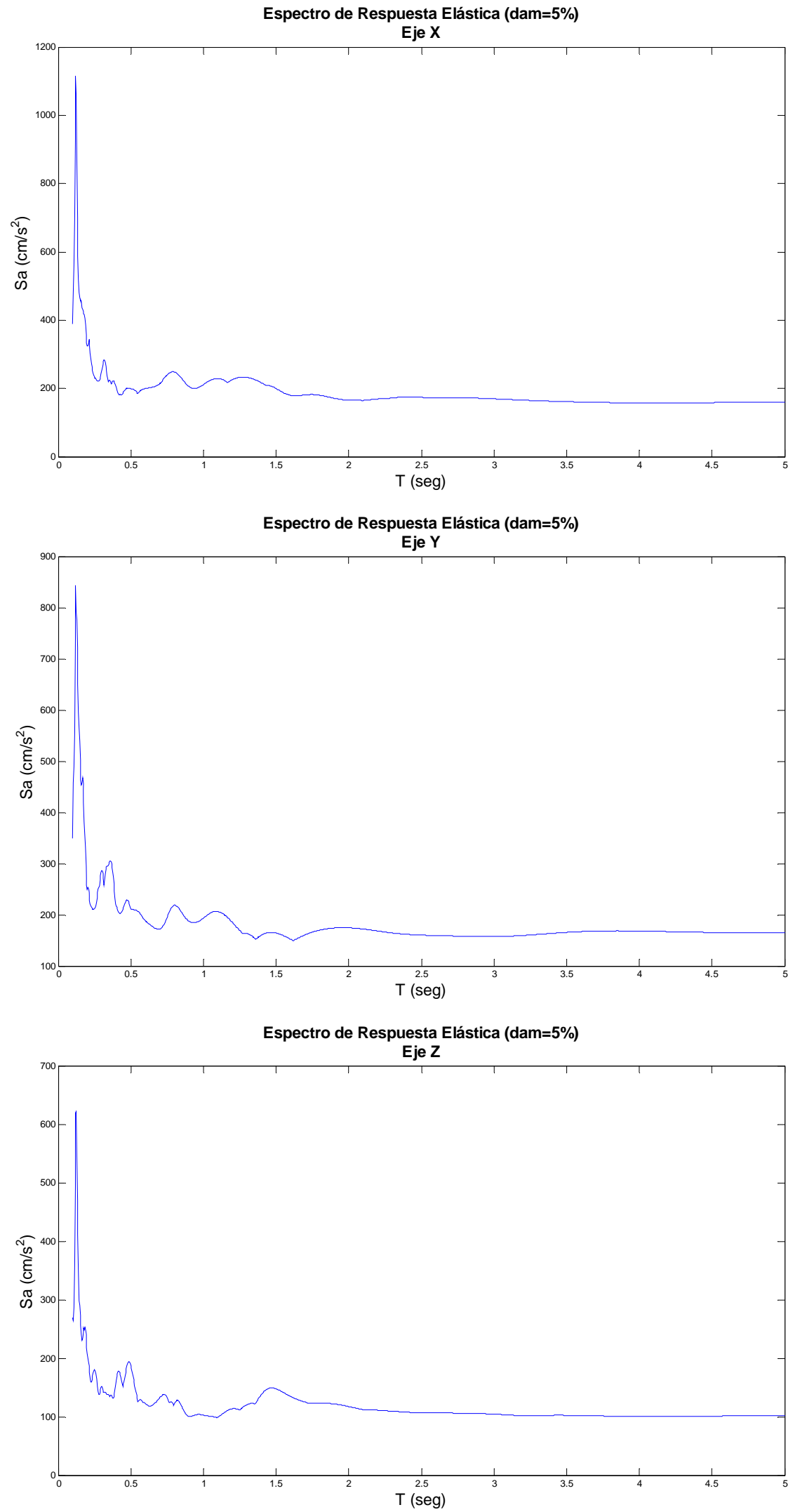

Figura 6.9. Espectros elásticos de respuesta de aceleración para una amortiguamiento del $5 \%$ 
Si se observan las dos simulaciones realizadas, podemos ver cómo al hacer que $C$ sea más bajo, y además más realista, siendo cercano a la unidad, los picos de los espectros se hacen menores y más lógicos para una terreno catalogado como Tipo I, es decir, roca o suelo muy duro. 


\section{APLICACIÓN AL CÁLCULO ESTRUCTURAL.}

En los puntos anteriores, hemos pasado desde la contextualización en el cálculo estructural de la acción sísmica representada mediante acelerogramas, a la consecución de acelerogramas sintéticos por el enfoque semiempírico ya fuesen por el modelado estadístico de Ordaz, et al., o por el modelado cinemático basado en los trabajos de Irikura. Con esto ya tenemos la herramienta para conseguir un acelerograma lo más realista posible, pero nos es necesario "escalarlos" para que cumplan la normativa.

La normativa exige que estos acelerogramas simulados deben de ajustarse a los espectros elásticos de respuesta de proyecto para un amortiguamiento del $5 \%$, ser coherentes, y para el caso de la NCSE-02, que el número de tríos de acelerogramas (dos horizontales, y uno vertical) sea de al menos cinco, siendo el promedio de los valores característicos obtenidos, la solicitación a adoptar.

Con lo cual, es bueno tener claro un esquema de trabajo que marque los pasos y comprobaciones a realizar a la hora de realizar un cálculo sísmico a partir de acelerogramas sintéticos.

\subsection{Esquema de trabajo.}

1. Estudio del entorno al lugar de emplazamiento del proyecto.

Se han de conocer del entorno tanto la parte normativa del cálculo sísmico, es decir, aceleración básica de cálculo, coeficiente de contribución y tipo de terreno, con lo que tenemos el espectro elástico de proyecto, como una idea general de la peligrosidad sísmica, del entorno tectónico y por tanto de los valores esperados en magnitud de los posibles eventos sísmicos .

2. Obtención de unos registros de eventos a usar como funciones de Green empíricas.

Estos registros tienen que ser representativos de los sistemas de fallas potencialmente peligrosos en el entorno, y de una calidad mínima para que a la hora de generar el registro simulado, este no se vea influenciado por una mala calidad del registro.

También tienen que estar registrados en estaciones lo más próximas a la zona de emplazamiento del proyecto, para que las particularidades de los efectos del sitio no hagan cometer errores. 
Los datos necesarios, además de los registros en los tres ejes, para el evento función de Green empírica son:

- Datos generales del terreno: densidad y velocidad de ondas S

- Coordenadas terrestres del hipocentro en UTM

- Coordenadas terrestres de la estación en UTM

- Magnitud momento, frecuencia de esquina y caída de esfuerzos

- Mecanismo focal o al menos datos de acimut y buzamiento de la falla (modelado cinemático)

El resto de datos de la función de Green empírica están relacionados con estos e implementados en cada uno de los modelados realizados.

3. Fijar la magnitud momento y frecuencia de esquina del evento objetivo. Realizar la simulación.

Como los espectros de la norma se expresan en aceleraciones, y con los métodos de simulación lo que fijamos son los momentos sísmicos, debemos de hacer un proceso iterativo variando la magnitud momento y la frecuencia de esquina para ajustarlo al espectro de respuesta de la normativa.

El tomar una magnitud momento u otra, puede ser un proceso más o menos intuitivo, que se basa en datos publicados. Pero el problema está en fijar la frecuencia de esquina o la caída de esfuerzos para un evento objetivo del cual no tenemos datos certeros, como es el caso de las zonas de intensidad moderada, donde no hay registros de eventos grandes.

Courboulex et al. (2010) estudia el problema de calibrar la caída de esfuerzos del evento objetivo y por tanto del parámetro $C$ de los métodos de simulación. Y realiza las simulaciones para un rango bastante amplio de posibles valores de $C$, en un intervalo de 1.4 a 26 para el caso de la Isla de Guadalupe, en las Antillas francesas. También Courboulex y Salichon en 2009 habían realizado simulaciones con unos valores de $C$ de entre 1 y 18.6 para un evento en la zona de Niza.

Lo lógico sería empezar por un valor de $C$ la unidad, e ir incrementando para cada iteración que se realice o incrementar el valor de la magnitud momento, pero sin llegar a valores tan extremos como en estos casos comentados. Una tabla que para cada valor de magnitud momento de evento objetivo fijada nos dé la correspondencia entre posibles valores de $C$ y sus correspondientes valores de frecuencia de esquina para el evento objetivo podría ser de gran utilidad.

Además de estos parámetros fijados para el evento objetivo, no debemos olvidar que para el modelado cinemático hay que definir de manera determinista el origen de la ruptura en el plano de falla del evento objetivo. Como ya se dijo cuando se explicó la implementación del método basado en Irikura, los parámetros que contralan esto son los parámetros $a$ y $b$. 
a controla las coordenadas horizontales de la posición del nuevo hipocentro en función de la dirección y sentido del acimut, y $b$ controla la coordenada vertical en función del buzamiento del plano de falla.

4. Comparar los espectros elásticos de respuesta para un amortiguamiento del $5 \%$ de cada uno de los ejes con los respectivos espectros elásticos de proyecto determinados por la norma. Si son similares, el registro simulado es válido, si no volvemos al punto 3, a fijar una magnitud momento y frecuencia de esquina nuevas para realizar una nueva simulación.

5. Introducción de la acción en un programa de cálculo.

\subsection{Simulación de terremotos en la zona de Granada}

Vamos a aplicar ahora el esquema de trabajo para varios casos en la zona de Granada, zona de sismicidad moderada, en la cual se sabe que ha habido eventos sísmicos importantes, pero que no se tiene ninguno registrado por ser evento antiguos.

Vamos a suponer que se van a proyectar unos edificios de importancia normal $(\rho=1.0)$ en las siguientes ubicaciones, con su asignación de tipo de terreno:

- Zona universitaria de Cartuja (Granada): Terreno tipo I (complejo Alhambra) $C=1.0$

- Zona de Facultad de Ciencias (Granada): Terreno tipo III $C=1.6$

- Zona del Cementerio de Santa Fé: Terreno tipo IV $C=2.0$

La aceleración básica de cálculo será de $0.23 \mathrm{~g}$ para Granada y 0.24g para Santa Fé, ambas con un coeficiente de contribución de valor $K=1.0$.

Los valores del coeficiente de amplificación, $S$, para las distintas zonas, será por tanto:

- Zona universitaria de Cartuja (Granada): $S=0.8866$

- Zona de Facultad de Ciencias (Granada): $S=1.1588$

- Zona del Cementerio de Santa Fe: $S=1.3203$

\subsubsection{Contexto geológico y sismotectónico.}

La zona de la depresión de Granada se encuentra enclavada dentro de las Cordilleras Béticas, las cuales, forman junto a las Cordilleras de Rif del norte de África, el segmento más occidental del orógeno alpino mediterráneo. Estas dos cordilleras, separadas en la actualidad por la cuenca neógena de Alborán, se localizan entre dos zócalos hercínicos, el Ibérico al norte y el Africano al sur. 
Las Cordilleras Béticas se formaron como consecuencia del régimen compresivo que comenzó a finales del Cretácico y en ellas se pueden distinguir distintos dominios o zonas, siendo las mas importantes, de norte a sur, las Zonas Externas y las Zonas Internas. Estas zonas, separadas y diferenciadas por un contacto tectónico, presentan además un origen paleogeográfico distinto.

Además de estas dos grandes zonas, existen otros dominios entre los que destacan las depresiones post-orogénicas terciarias, rellenas de materiales terciarios y cuaternarios procedentes de la erosión de los relieves circundantes.

La zona de la depresión de Granada es una de estas cuencas post-orogénicas que se sitúa de manera discordante sobre el contacto entre las Zonas Externas y las Zonas Internas. La historia geológica de la depresión de Granada comienza a mediados del Mioceno, cuando se reanuda la sedimentación tras un periodo marcado por importantes inestabilidades tectónicas.

En el Mioceno superior se produce una transgresión marina, así como una elevación de los relieves, que originan una importante avalancha conglomerática que se adosa a los bordes de la cuenca. En el Tortoniense superior esta cuenca marina se continentaliza y es sustituida por una cuenca endorreica de sedimentación lacustre.

En el Mioceno terminal debió ocurrir un rejuvenecimiento del relieve y/o un cambio climático importante que condicionó la instauración de un régimen fluvio - lacustre con importantes aparatos fluviales provenientes de las sierras circundantes. En el Pleistoceno medio y superior se reactivaron y/o crearon fracturas de importante salto que fueron y siguen siendo en la actualidad rellenadas por los aportes del río Genil y sus afluentes.

En esta zona de la depresión de Granada y en sus zonas aledañas aparece una actividad sísmica moderada, con eventos superficiales en su mayoría, algunos intermedios y unos pocos de profundidades del entorno a los $650 \mathrm{~km}$. Esta zona es la zona de mayor sismicidad de toda España, con conocidos terremotos históricos de importantes intensidades.

Se han realizado estudios sobre las magnitudes esperadas en esta zona, como se puede ver en la Fig. 7.1., en la que se muestra una gráfica que contrapone la magnitud $M$ con el $\log N$ siendo $N$ el número de eventos por año con una magnitud superior a $M$ (Miguel de el al. (1988)). Según estos estudios el periodo de retorno aproximado para eventos con magnitudes 5, 6 y 7 sería respectivamente 10, 100, y 1000 años.

Además de este estudio, Sanz de Galdeano, Peláez y López (2003) publicaron un catálogo de las fallas activas en la zona de la depresión de Granada y aledaños. En este catálogo se pueden obtener datos de magnitudes momento máximas que se pueden dar para eventos en las distintas fallas activas de la zona.

Otros datos sobre el entorno que podemos encontrar en la bibliografía, son los parámetros de la corteza, que aparecen en la publicación de Morales et al. (1996), donde podemos 
encontrar la velocidad de las ondas $\mathrm{S}$ y la densidad en función de la profundidad, aplicables a esta zona (Tabla 7.1).

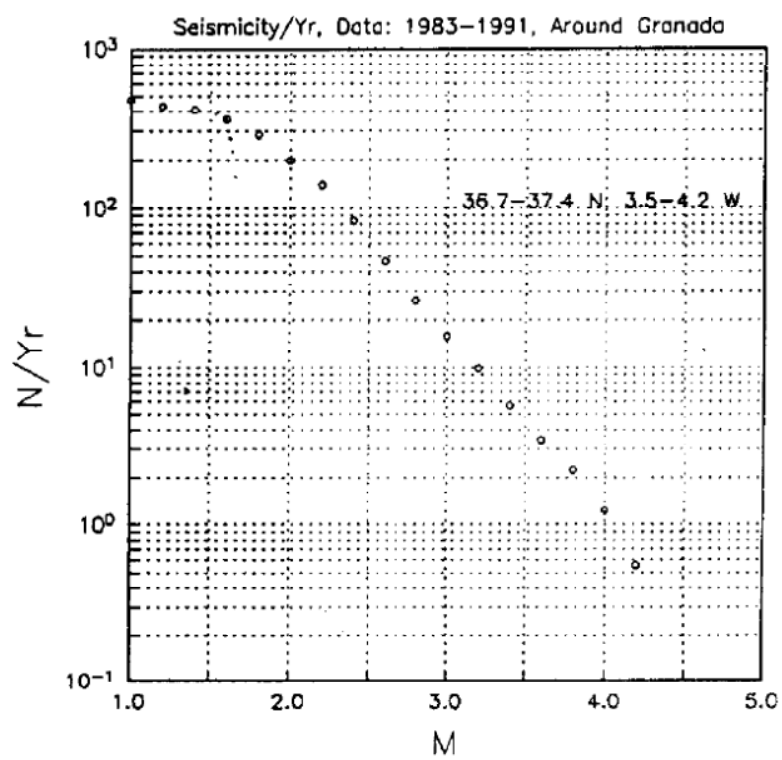

Figura 7.1. Magnitud vs. $\log N$

Tabla 7.1. Parámetros de la corteza terrestre para el sur de España

\begin{tabular}{|c|c|c|}
\hline $\begin{array}{c}\text { Profundidad } \\
(\mathrm{km})\end{array}$ & $\begin{array}{c}\text { Velocidad de ondas S } \\
(\mathrm{Km} / \mathrm{s})\end{array}$ & Densidad $\left(\mathrm{gr} / \mathrm{cm}^{3}\right)$ \\
\hline 3.5 & 2.25 & 2.7 \\
\hline 7.5 & 3.50 & 2.8 \\
\hline 39.0 & 4.23 & 2.9 \\
\hline$\infty$ & 4.50 & 3.2 \\
\hline
\end{tabular}

\subsubsection{Acción sísmica en un edificio de importancia normal ubicado en la zona universitaria de Cartuja (Granada)}

\subsubsection{Espectros de respuesta elástica de la normativa.}

La zona de Cartuja en Granada, tenemos los siguientes valores de la normativa, para el cálculo de los espectros de respuesta elástica para cada uno de los ejes. $a_{b}=0.23 \mathrm{~g}$, $\rho=1.0, \quad K=1.0, \quad S=0.8866$ y $C=1.0$, con lo que la aceleración de cálculos es $a_{c}=S \cdot \rho \cdot a_{b}=200.04 \mathrm{~cm} / \mathrm{s}^{2}$.

Los espectros elásticos de proyecto por eje son los que se muestran a continuación en la Fig.7.2. 

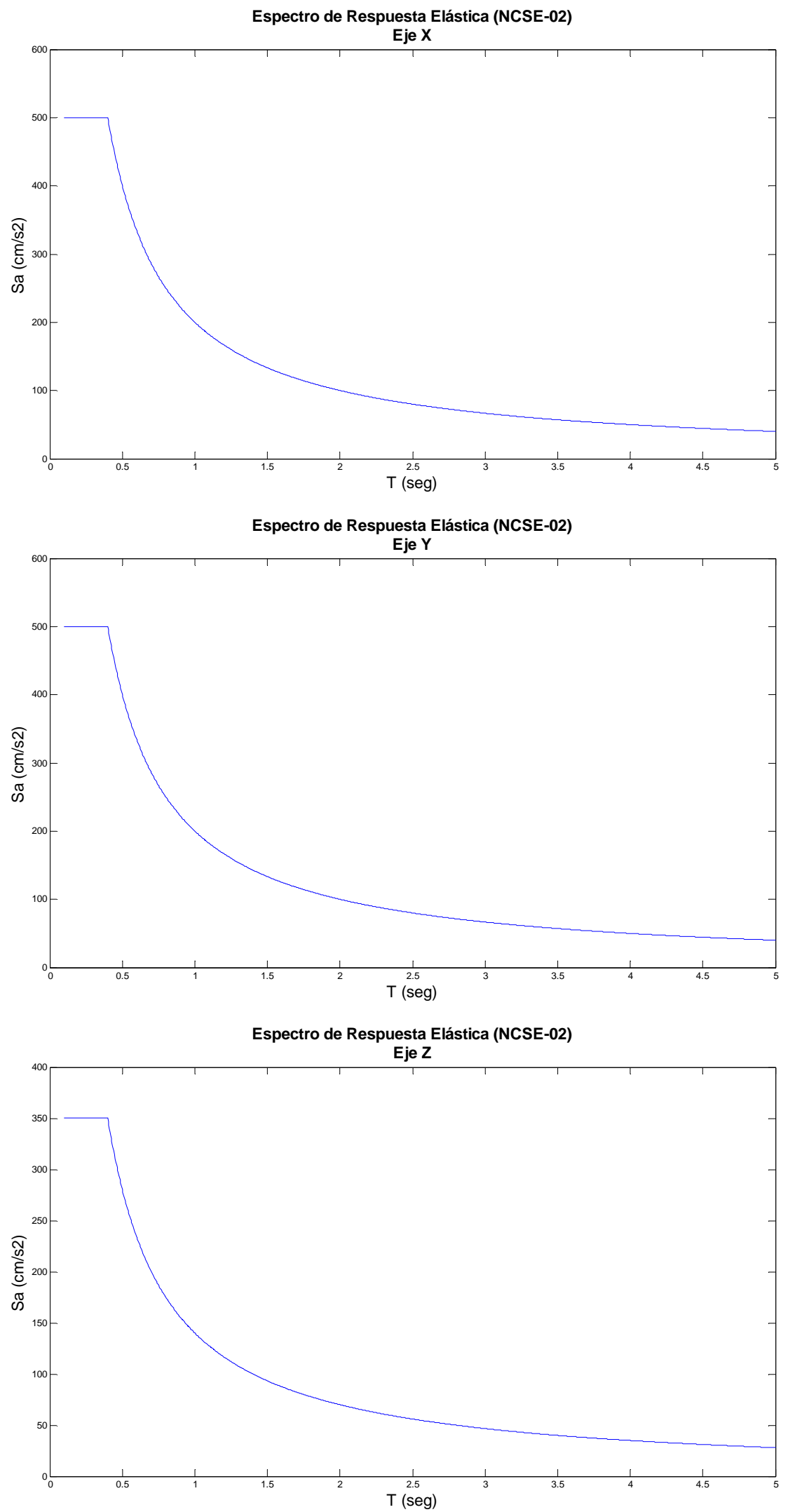

Figura 7.2. Espectros de respuesta elásticos según la normativa según los ejes 


\subsubsection{Simulación por el modelo estadístico.}

Usando el terremoto del 24 de Febrero de 1997 de Agrón, que se puede ver en el Anejo 1, realizamos una simulación del modelo estadístico, con una magnitud momento esperada 6.0 y una frecuencia de esquina esperada de $0.38 \mathrm{~Hz}$, para un seísmo registrado en el observatorio de Cartuja (Granada).

Para el modelado estadístico tenemos la siguiente tabla de datos de la simulación (Tabla 7.2.):

Tabla 7.2. Datos de la simulación

\begin{tabular}{|c|c|c|c|}
\hline Datos del Medio & & & \\
\hline Velocidad de Ondas S & \multicolumn{2}{|r|}{3.5} & \\
\hline Velocidad de Ruptura & \multicolumn{2}{|r|}{2.975} & \\
\hline Datos de los Eventos & Evento EGF & Evento Objetivo & \\
\hline Magnitud Momento & 4.3 & 6 & \\
\hline Momento Sísmico & $2.94 \mathrm{E}+15$ & $1.12 \mathrm{E}+18$ & \\
\hline Frecuencia de Esquina & 1.2 & 0.38 & \\
\hline Caída de Esfuerzos & 11 & 122.0558 & \\
\hline Factor C & \multicolumn{2}{|c|}{12.1188} & \\
\hline Factor $\eta$ & \multicolumn{2}{|r|}{99} & \\
\hline Factor $\xi$ & \multicolumn{2}{|c|}{3.8376} & \\
\hline
\end{tabular}

La función temporal equivalente de la fuente en este caso es la que se muestra en la Fig.7.3.:

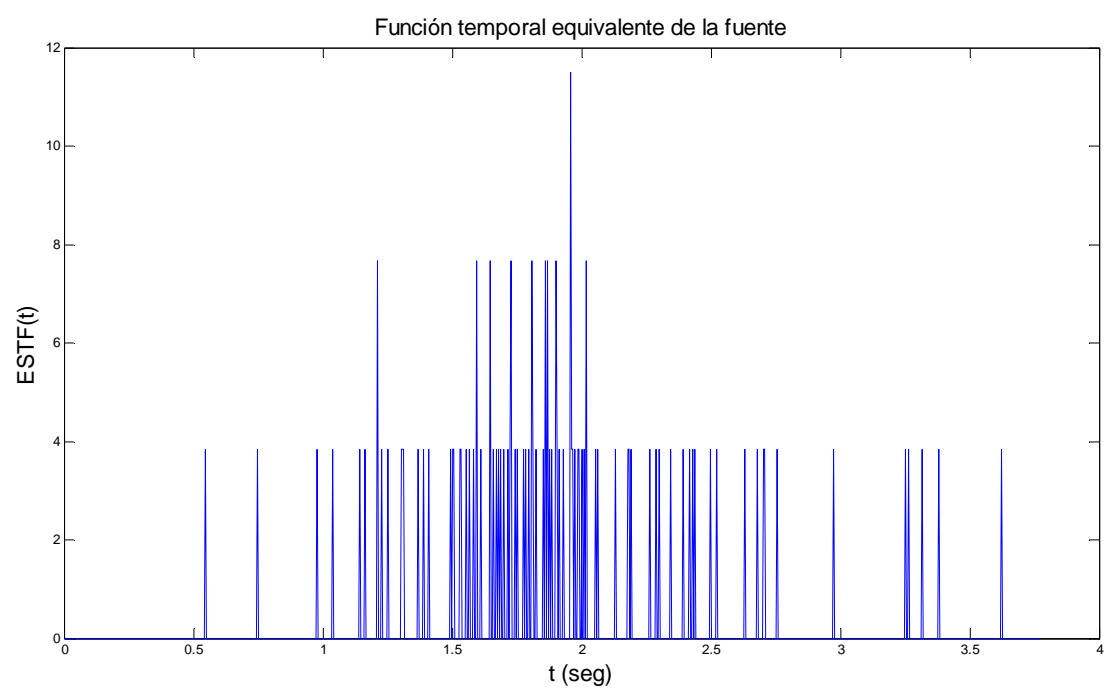

Figura 7.3. Función temporal equivalente de la fuente

De donde realizando la convolución, obtenemos los acelerogramas del evento simulado, así como los espectros elásticos de respuesta de aceleraciones para un amortiguamiento 
del 5\% comparados con los espectros elásticos de proyecto dados por la normativa (Fig.7.4. y Fig.7.5.).
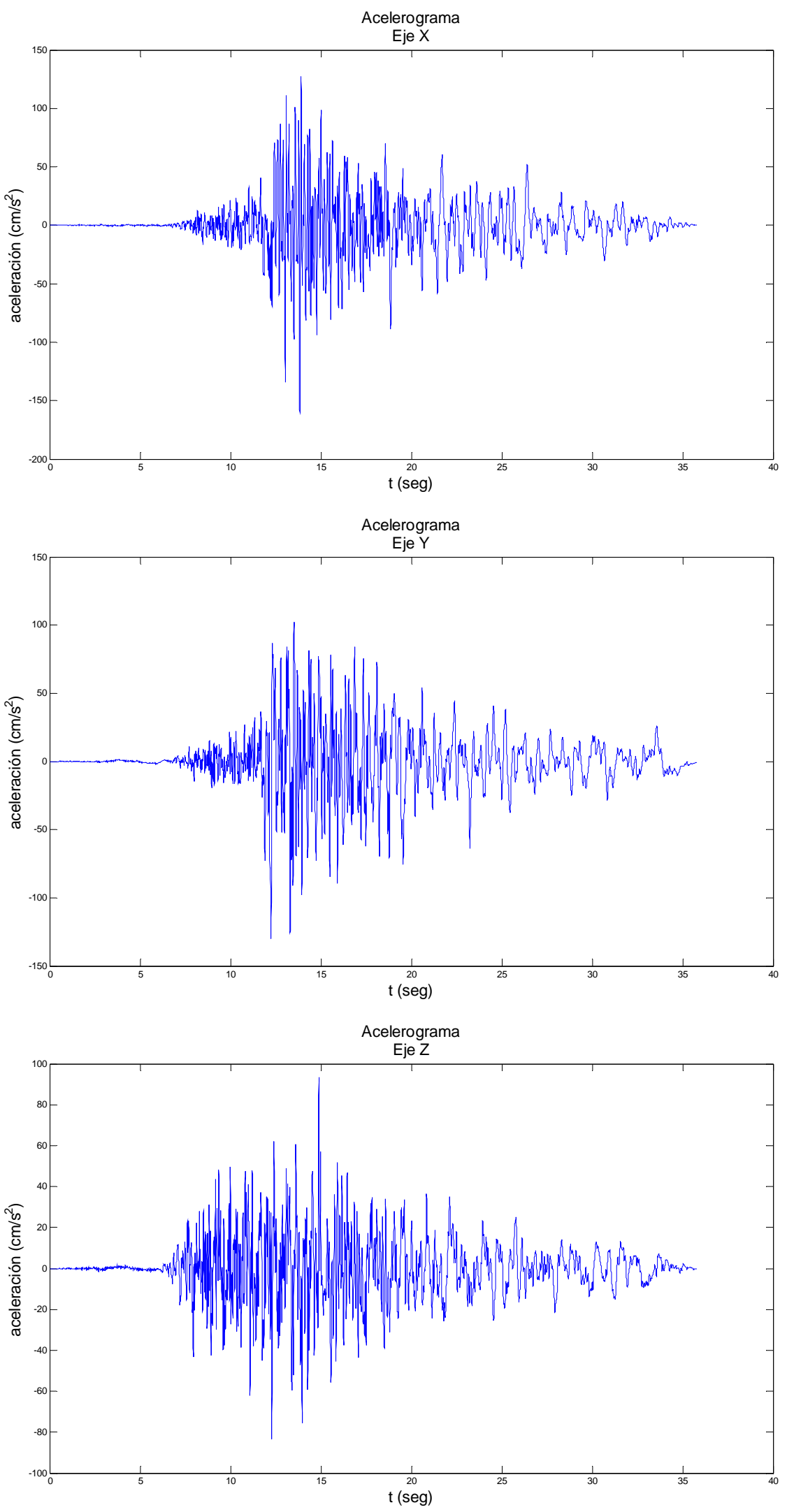

Figura 7.4. Acelerogramas simulados 

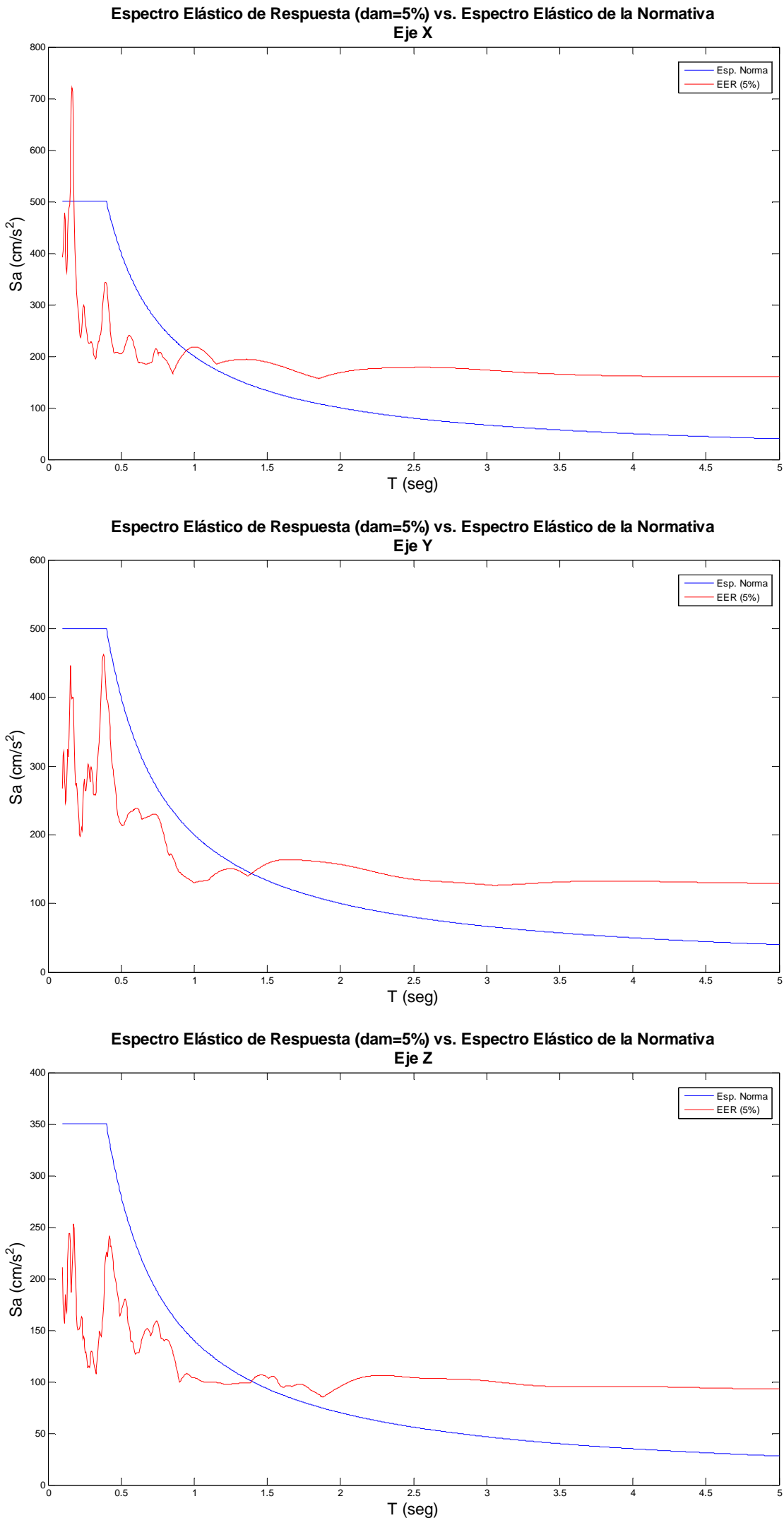

Figura 7.5. Espectros de evento simulado comparados con los espectros de la normativa 
Al igual que en Morales et al. (1996), para terrenos tipo roca o suelo duro el espectro de proyecto se queda del lado de la seguridad, y como se puede observar se ha tenido que ir a un valor de $C$ muy alto para acercarlo lo más posible o incluso sobrepasarlo muy poco para el eje $x$.

\subsubsection{Simulación por el modelo cinemático:}

Para el mismo terremoto función de Green empírica, misma estación y mismos parámetros objetivo, realizamos una simulación con el método de Irikura, obteniendo los siguientes resultados en datos, función temporal equivalente a la fuente, acelerogramas y espectros comparados (Tabla 7.3., Fig.7.6., Fig.7.7. y Fig.7.8.).

Tabla 7.3. Datos de la simulación

\begin{tabular}{|c|c|c|c|}
\hline Datos del Medio & & & \\
\hline Velocidad de Ondas S & \multicolumn{2}{|r|}{3.5} & \\
\hline Velocidad de Ruptura & \multicolumn{2}{|c|}{2.975} & \\
\hline Datos de los Eventos & Evento EGF & Evento Objetivo & \\
\hline Magnitud Momento & 4.3 & 6 & \\
\hline Momento Sísmico & $2.94 \mathrm{E}+15$ & $1.12 \mathrm{E}+18$ & \\
\hline Frecuencia de Esquina & 1.2 & 0.38 & \\
\hline Caída de Esfuerzos & 11 & 122.0558 & \\
\hline Factor N & \multicolumn{2}{|r|}{4} & \\
\hline Factor C & \multicolumn{2}{|c|}{12.1188} & \\
\hline
\end{tabular}

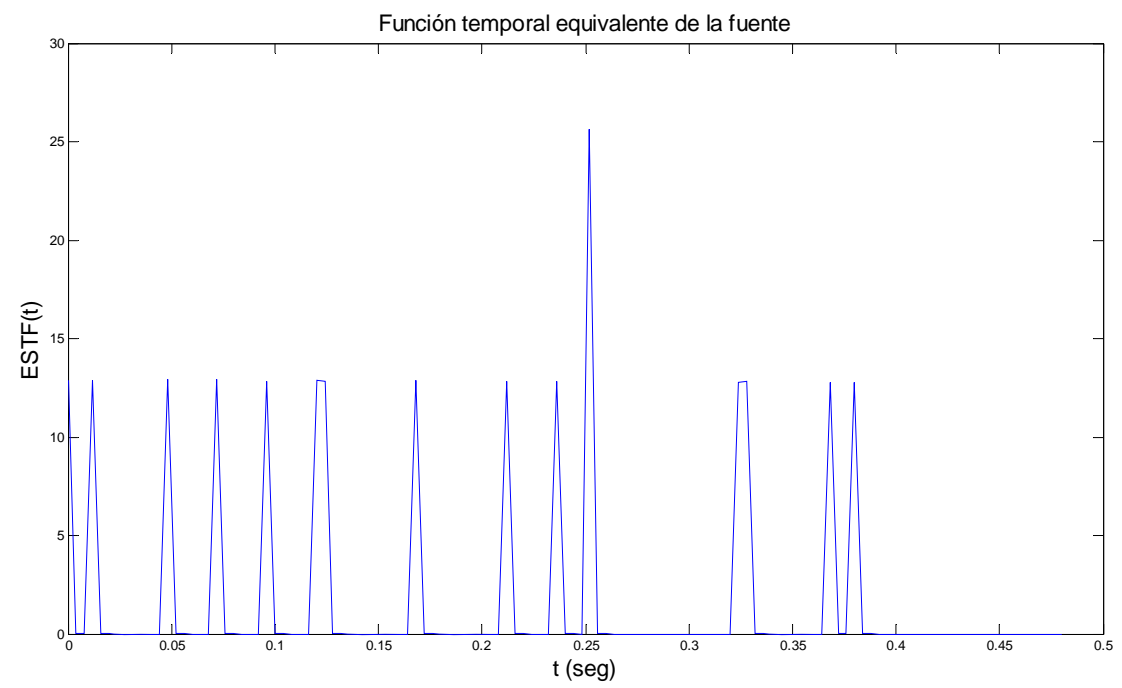

Figura 7.6. Función temporal equivalente de la fuente 

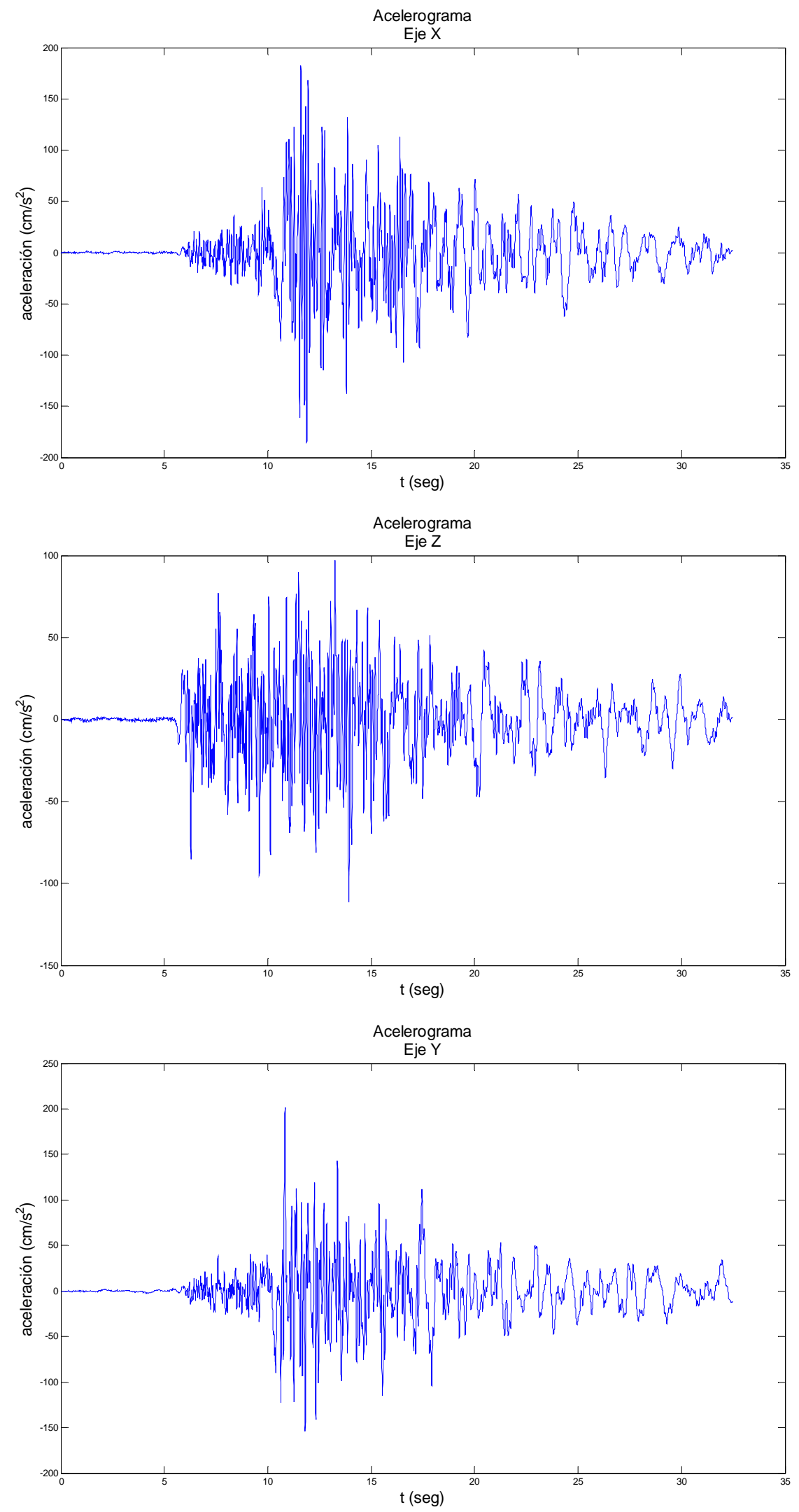

Figura 7.7. Acelerogramas simulados 

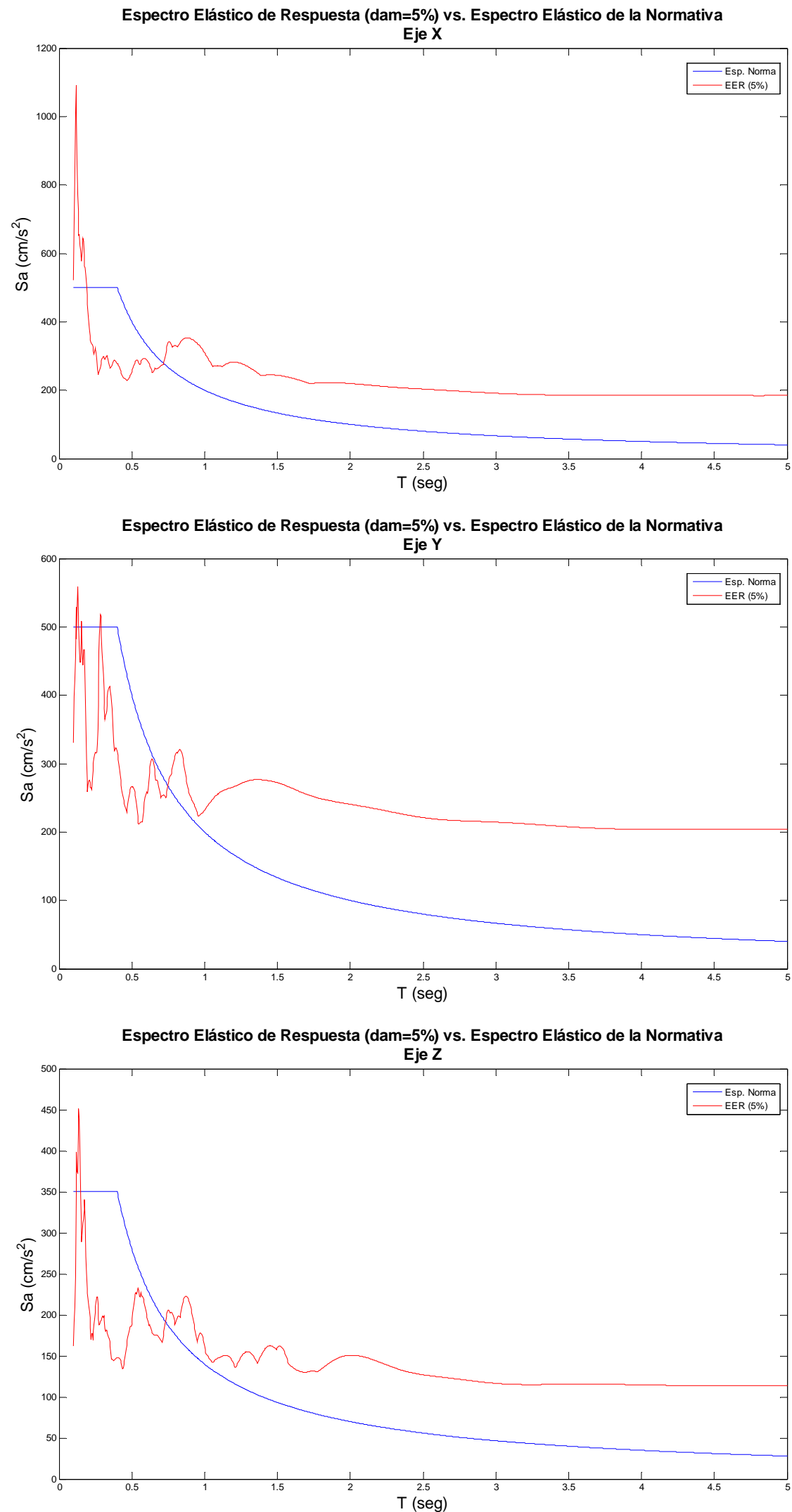

Figura 7.8. Espectros de evento simulado comparados con los espectros de la normativa 
Como se puede ver, los valores son más o menos similares para los dos modelados, reiterándose lo que se ha dicho, que el espectro de proyecto para roca dura está del lado de la seguridad, y para poder sobrepasarlo se ha tenido que ir a una relación de caída de esfuerzos muy grande. Hay que tener en cuenta que se trata de modelos con variables estocásticas, y cada simulación es distinta a la otra, pero en líneas generales se han obtenido valores similares para este caso.

\subsubsection{Acción sísmica en un edificio de importancia normal ubicado en la zona de la Facultad de Ciencias de la Universidad de Granada}

\subsubsection{Espectros de respuesta elástica de la normativa.}

La zona de Fuente Nueva en Granada, tenemos los siguientes valores de la normativa, para el cálculo de los espectros de respuesta elástica para cada uno de los ejes. $a_{b}=0.23 \mathrm{~g}$, $\rho=1.0, \quad K=1.0, \quad C=1.6$ y $S=1.1588$, con lo que la aceleración de cálculo es $a_{c}=S \cdot \rho \cdot a_{b}=261.46 \mathrm{~cm} / \mathrm{s}^{2}$. Los espectros elásticos de respuesta de proyecto por eje son los que se muestran en la Fig.7.9. 

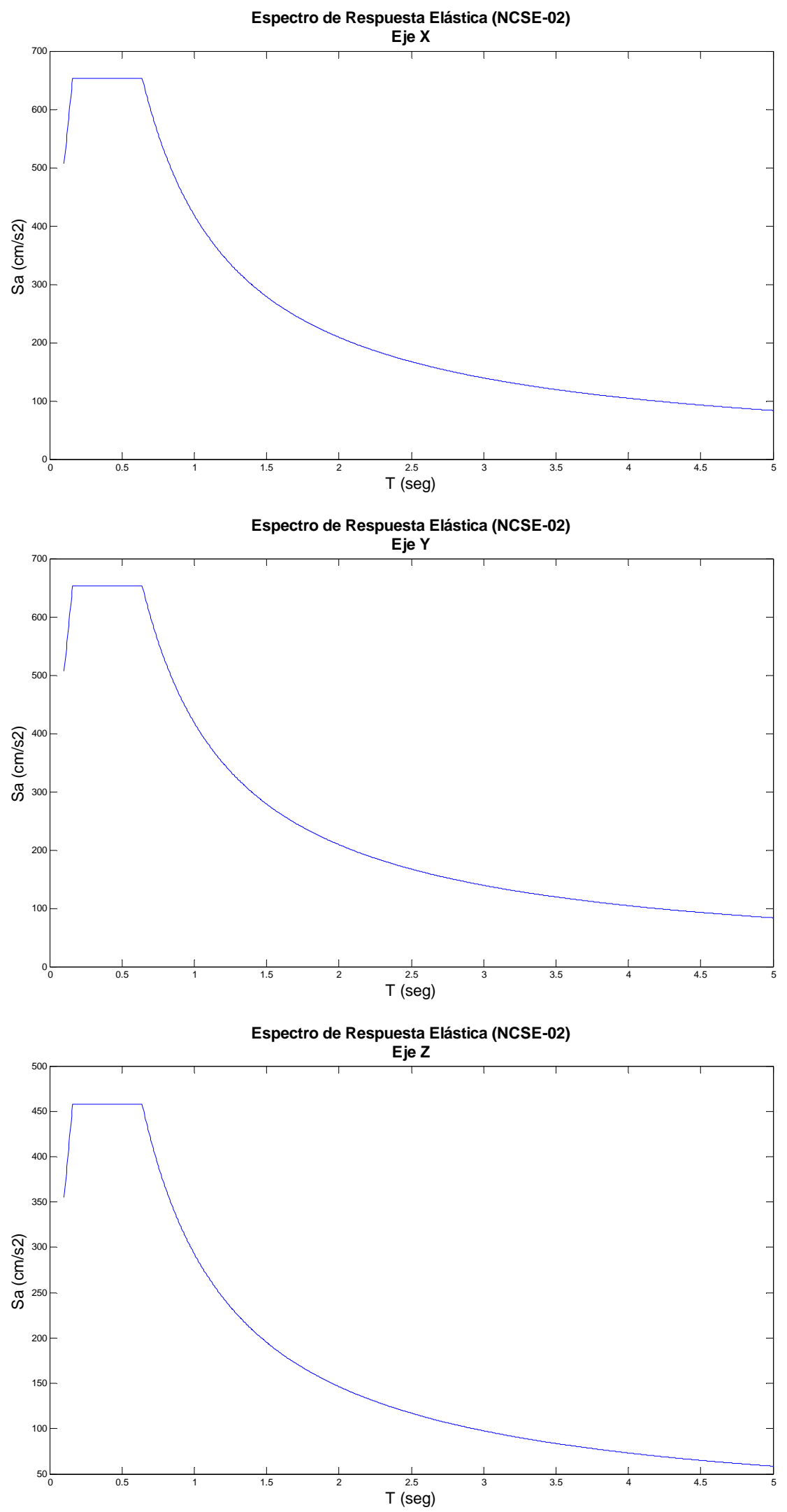

Figura 7.9. Espectros de respuesta elásticos de la normativa según los ejes 


\subsubsection{Simulación por el modelo estadístico:}

Usando el terremoto del 5 de Enero de 2007 de Sierra Elvira, que se puede ver en el Anejo 1, realizamos una simulación del modelo estadístico, con una magnitud momento esperada 5.8 y una frecuencia de esquina esperada de $0.25 \mathrm{~Hz}$, para un seísmo registrado en la Facultad de Ciencias (Granada) de la que obtenemos los siguientes resultados mostrados en la Tabla 7.4, Fig.7.10., Fig.7.11 y Fig.7.12.

Tabla 7.4. Datos de la simulación

\begin{tabular}{|c|c|c|c|}
\hline Datos del Medio & & & \\
\hline Velocidad de Ondas S & \multicolumn{2}{|r|}{3.5} & \\
\hline Velocidad de Ruptura & \multicolumn{2}{|c|}{2.975} & \\
\hline Datos de los Eventos & Evento EGF & Evento Objetivo & \\
\hline Magnitud Momento & 4.1 & 5.8 & \\
\hline Momento Sísmico & $1.58 \mathrm{E}+15$ & $5.62 \mathrm{E}+17$ & \\
\hline Frecuencia de Esquina & 1.4 & 0.25 & \\
\hline Caida de Esfuerzos & 8.6217 & 34.7558 & \\
\hline Factor C & \multicolumn{2}{|c|}{2.0267} & \\
\hline Factor $\eta$ & \multicolumn{2}{|c|}{983} & \\
\hline Factor $\xi$ & \multicolumn{2}{|c|}{0.3619} & \\
\hline
\end{tabular}

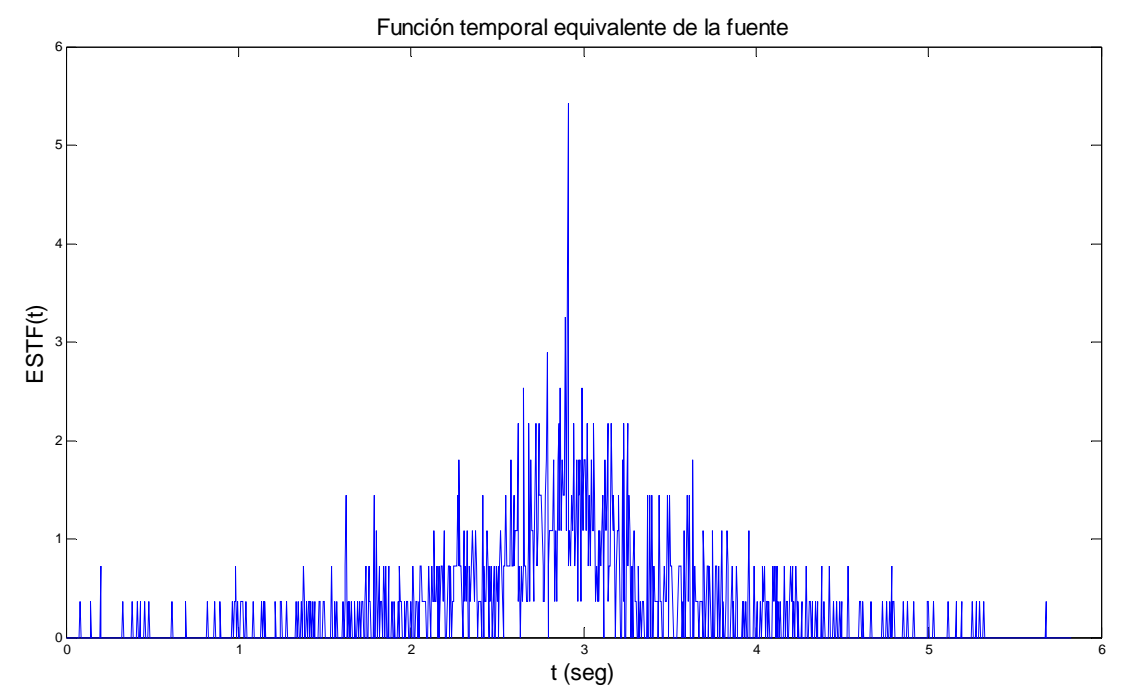

Figura 7.10. Función temporal equivalente de la fuente 

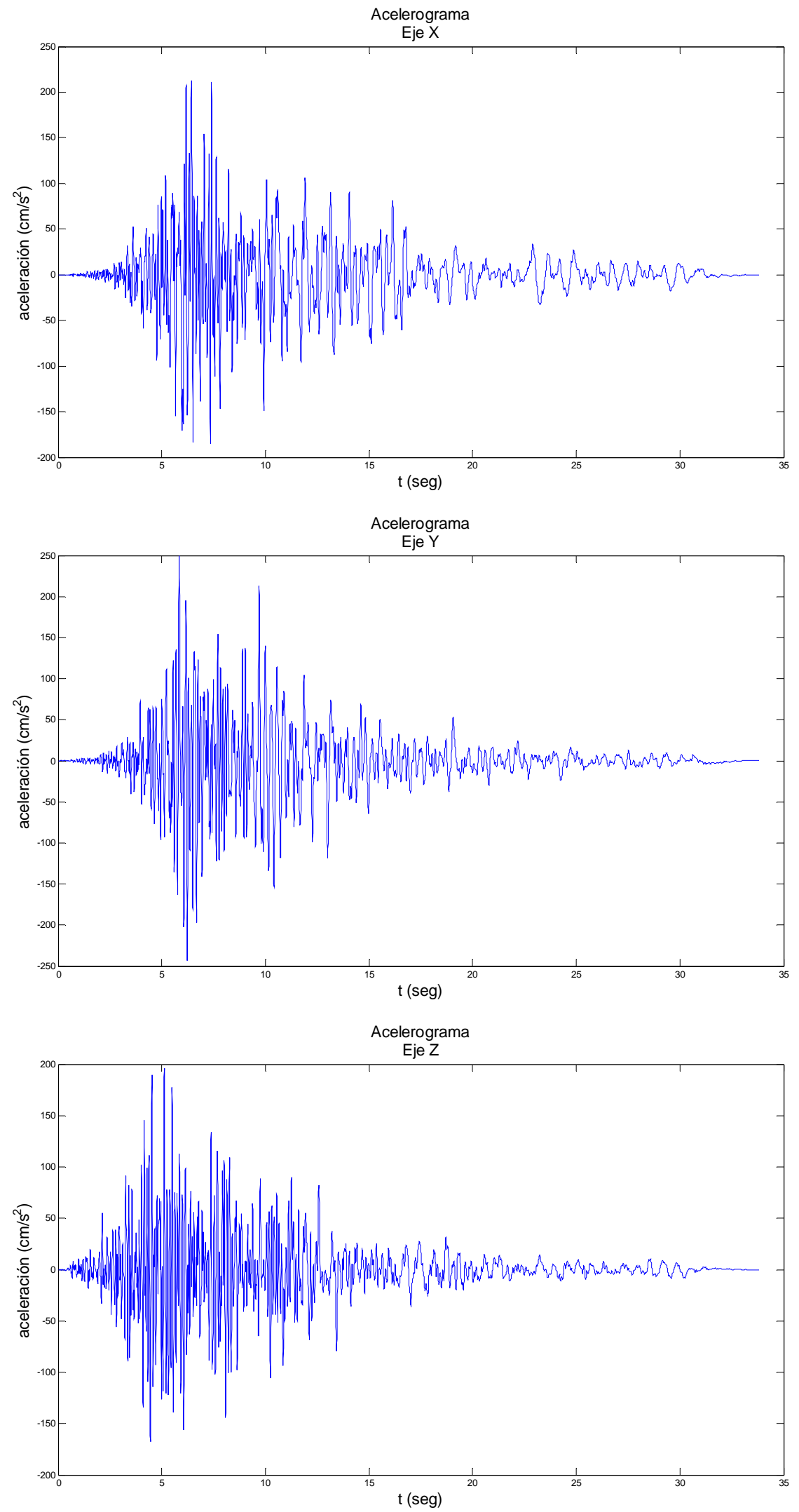

Figura 7.11. Acelerogramas simulados 
TFM

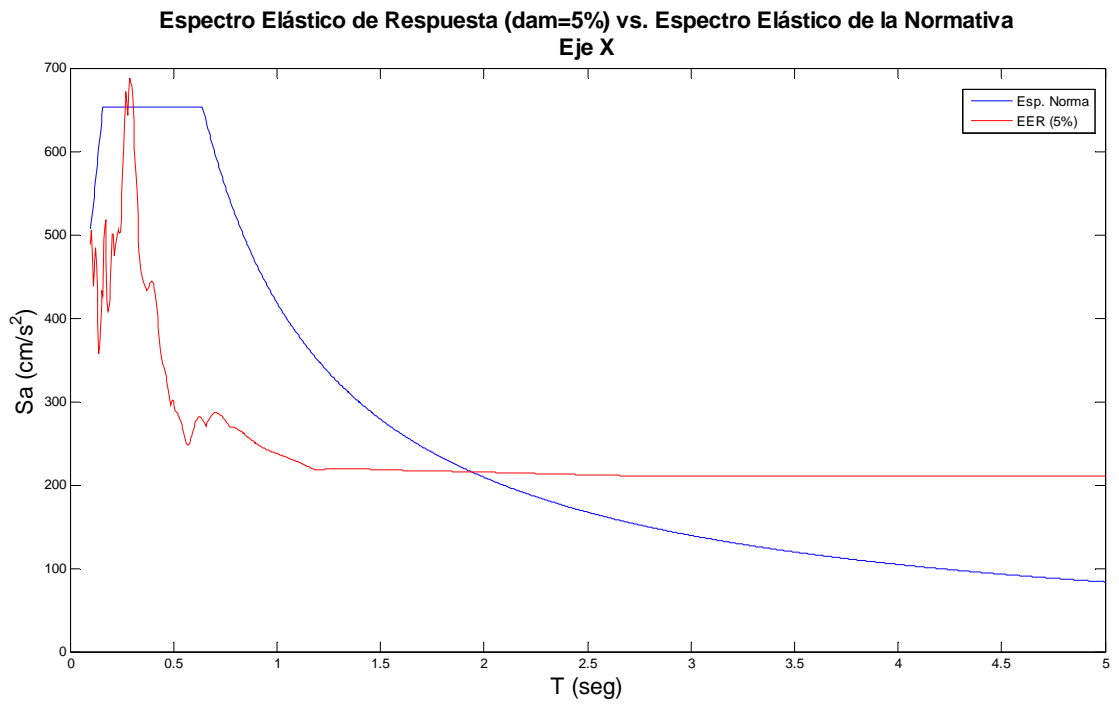

Espectro Elástico de Respuesta (dam=5\%) vs. Espectro Elástico de la Normativa

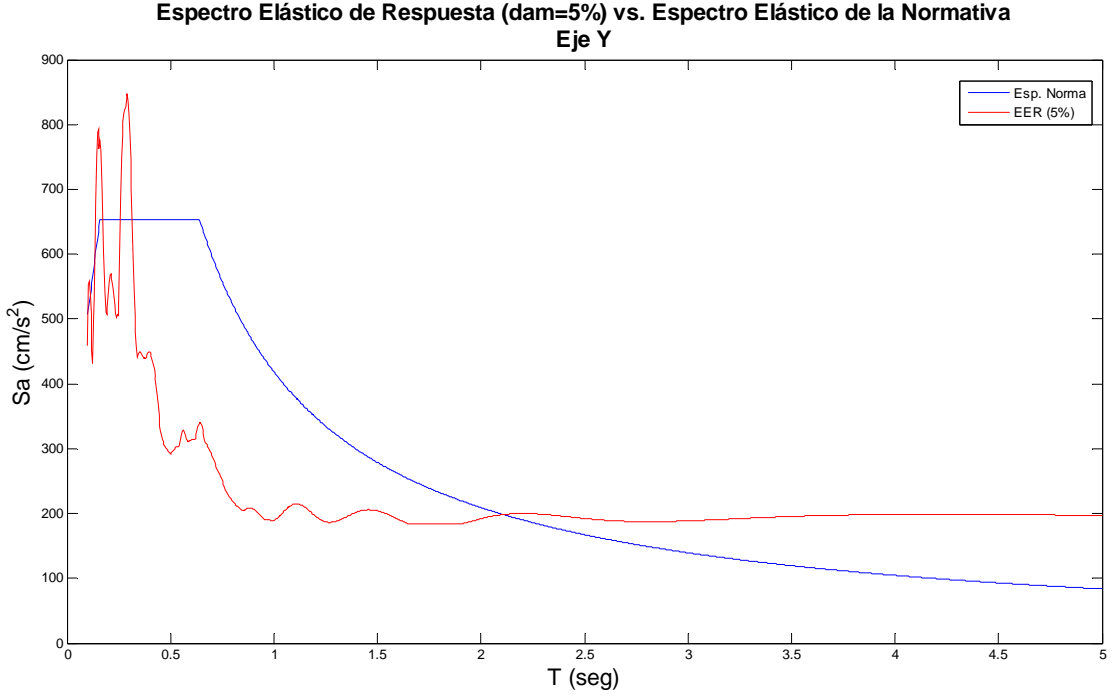

Espectro Elástico de Respuesta (dam=5\%) vs. Espectro Elástico de la Normativa Eje Z

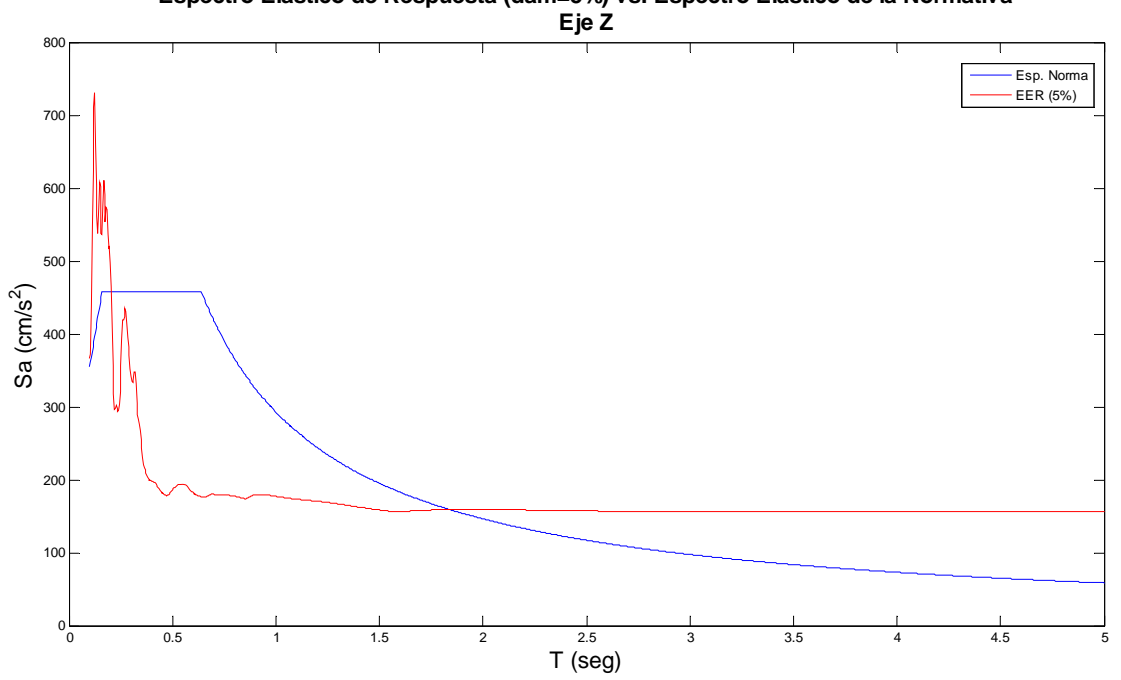

Figura 7.12. Espectros de evento simulado comparados con los espectros de la normativa

MÁSTER UNIVERSITARIO DE ESTRUCTURAS

105 
En estos espectros podemos ver que para periodos muy bajos, nos encontramos con picos importantes, lo cual puede ser debido a la presencia de suelos o por afección del propio edificio al acelerógrafo que registra.

\subsubsection{Simulación por el modelo cinemático.}

Variando un poco las magnitudes requeridas al evento objetivo, simulamos usando el mismo terremoto uno con una magnitud momento de 5.3 y una frecuencia de esquina del orden de $0.355 \mathrm{~Hz}$, para un evento registrado en la facultad de Ciencias de Granada.

La tabla de datos de la simulación (Tabla 7.5.), la función temporal equivalente de a la fuente (Fig.7.13), los acelerogramas simulados (Fig.7.14.) y espectros comparados por cada eje (Fig.7.15.) son los siguientes:

Tabla 7.5. Datos de la simulación

\begin{tabular}{|c|c|c|c|}
\hline Datos del Medio & & & \\
\hline Velocidad de Ondas S & \multicolumn{2}{|r|}{3.5} & \\
\hline Velocidad de Ruptura & \multicolumn{2}{|c|}{2.975} & \\
\hline Datos de los Eventos & Evento EGF & Evento Objetivo & \\
\hline Magnitud Momento & 4.1 & 5.3 & \\
\hline Momento Sísmico & $1.58 \mathrm{E}+15$ & $1.00 \mathrm{E}+17$ & \\
\hline Frecuencia de Esquina & 1.4 & 0.355 & \\
\hline Caida de Esfuerzos & 8.6217 & 8.8694 & \\
\hline Factor N & \multicolumn{2}{|r|}{4} & \\
\hline Factor C & \multicolumn{2}{|c|}{1.0319} & \\
\hline
\end{tabular}

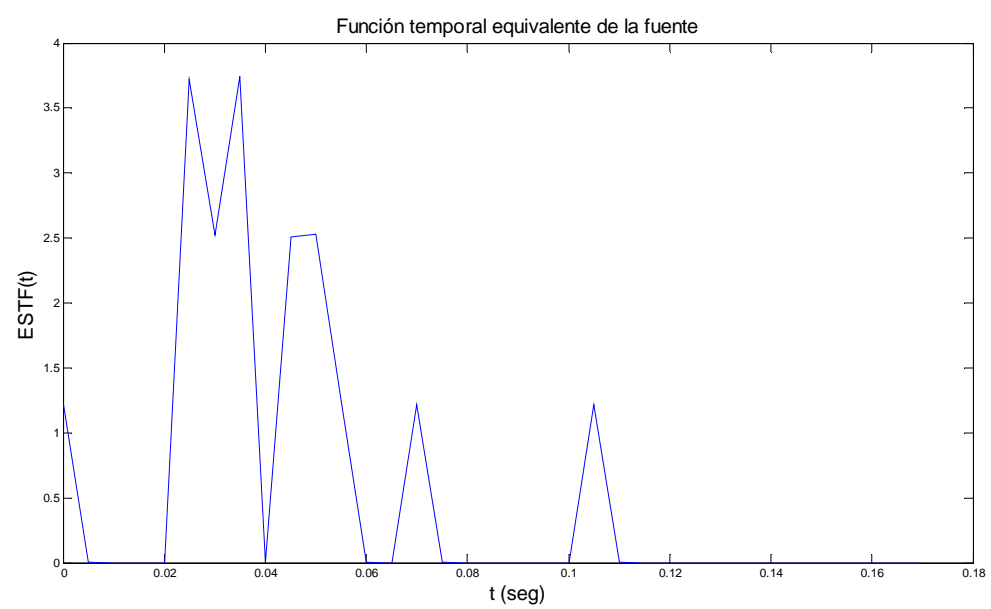

Figura 7.13. Función temporal equivalente de la fuente 

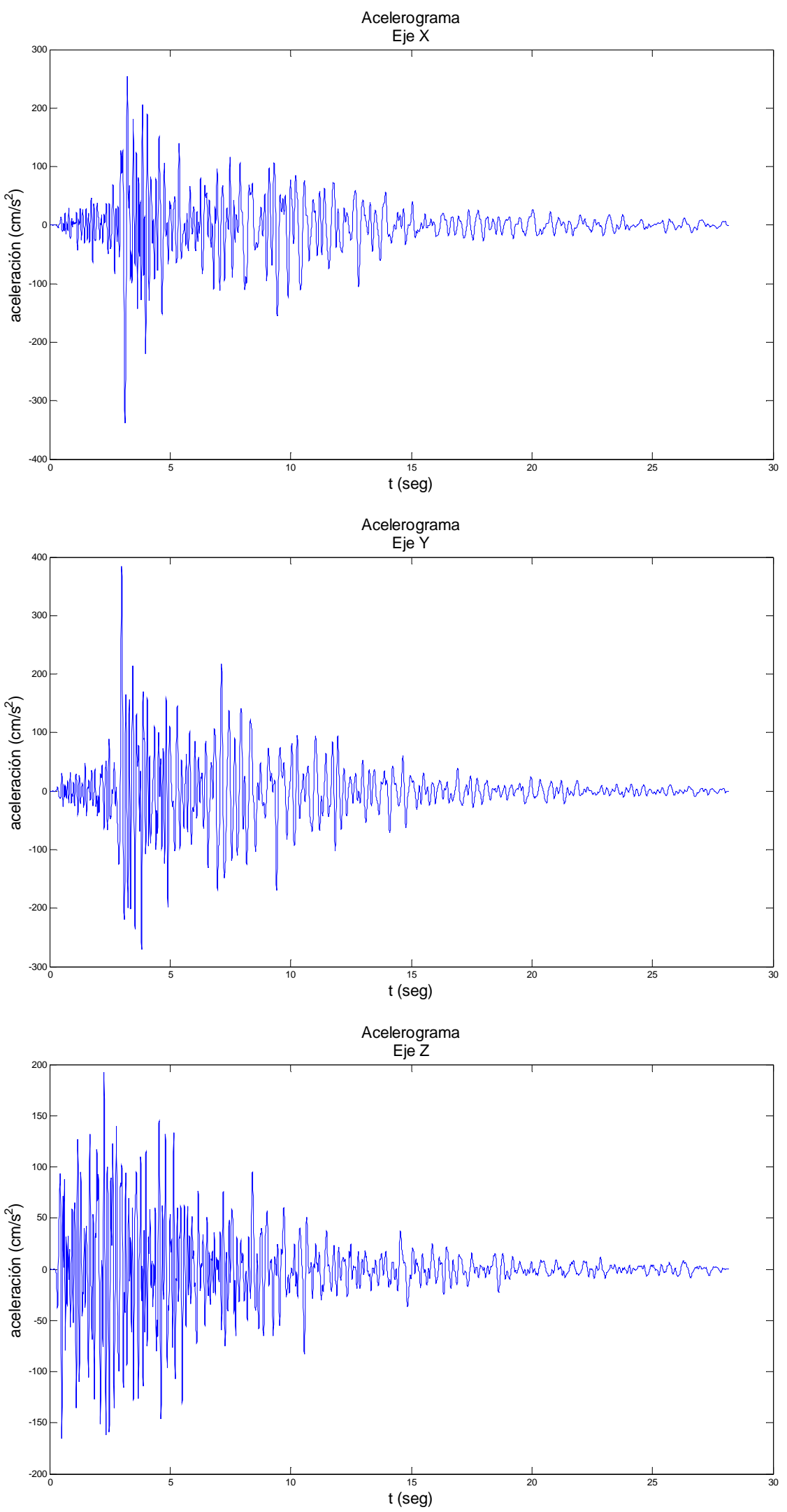

Figura 7.14. Acelerogramas simulados 

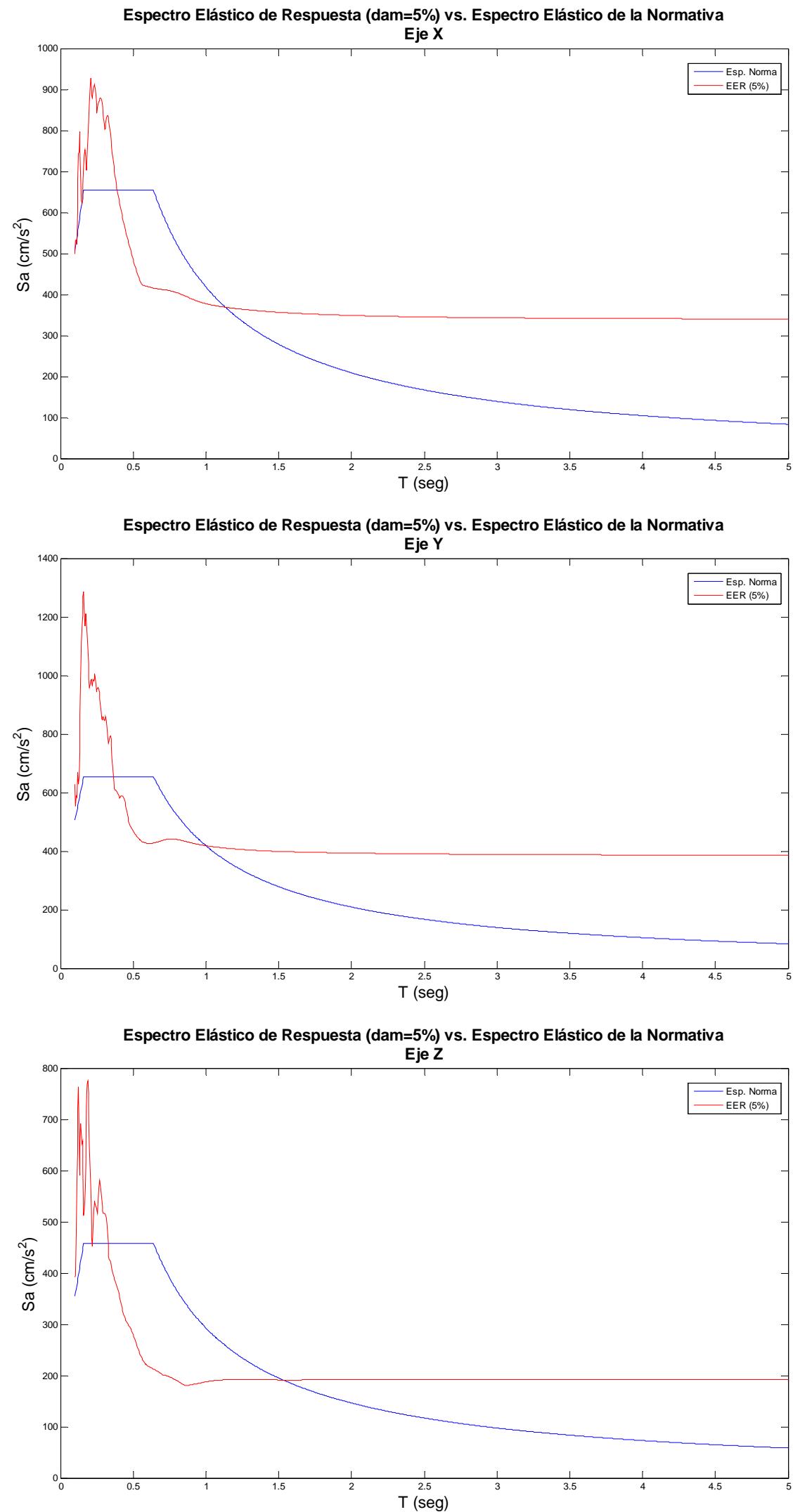

Figura 7.15. Espectros de evento simulado comparados con los espectros de la normativa 
Como se puede observar, aun habiendo bajado la magnitud momento esperada, y obteniendo una relación $C$ de valor casi la unidad, se obtienen picos muy importantes para este tipo de suelo blando, mayores incluso que para el modelado estadístico O.S.A. Esto puede ser debido a la tipología del terreno, a la afección de la instalación del acelerógrafo en los registros, o al número de subeventos tenidos en cuenta.

El modelado cinemático basado en los postulados de Irikura toma un número de subeventos mucho menor que en el modelado estadístico, por lo que tiene que multiplicarse por un factor de escalado (en el modelado cinemático $C$ ) mucho mayor, que en el modelado estadístico $(\xi)$, lo cual puede generar distorsiones más importantes al utilizar registros a modo de función de Green empírica con algún tipo de distorsiones o ruido.

\subsubsection{Acción sísmica en un edificio de importancia normal ubicado en Santa Fé (Granada)}

\subsubsection{Espectros de respuesta elástica de la normativa:}

La zona del Cementerio de Santa Fe (Granada), tenemos los siguientes valores de la normativa, para el cálculo de los espectros de respuesta elástica para cada uno de los ejes. $a_{b}=0.24 g, \rho=1.0, K=1.0, C=2.0$ y $S=1.3203$, con lo que la aceleración de cálculos es $a_{c}=S \cdot \rho \cdot a_{b}=310.85 \mathrm{~cm} / \mathrm{s}^{2}$. Los espectros elásticos de respuesta de proyecto se muestran a continuación en la Fig.7.16.: 

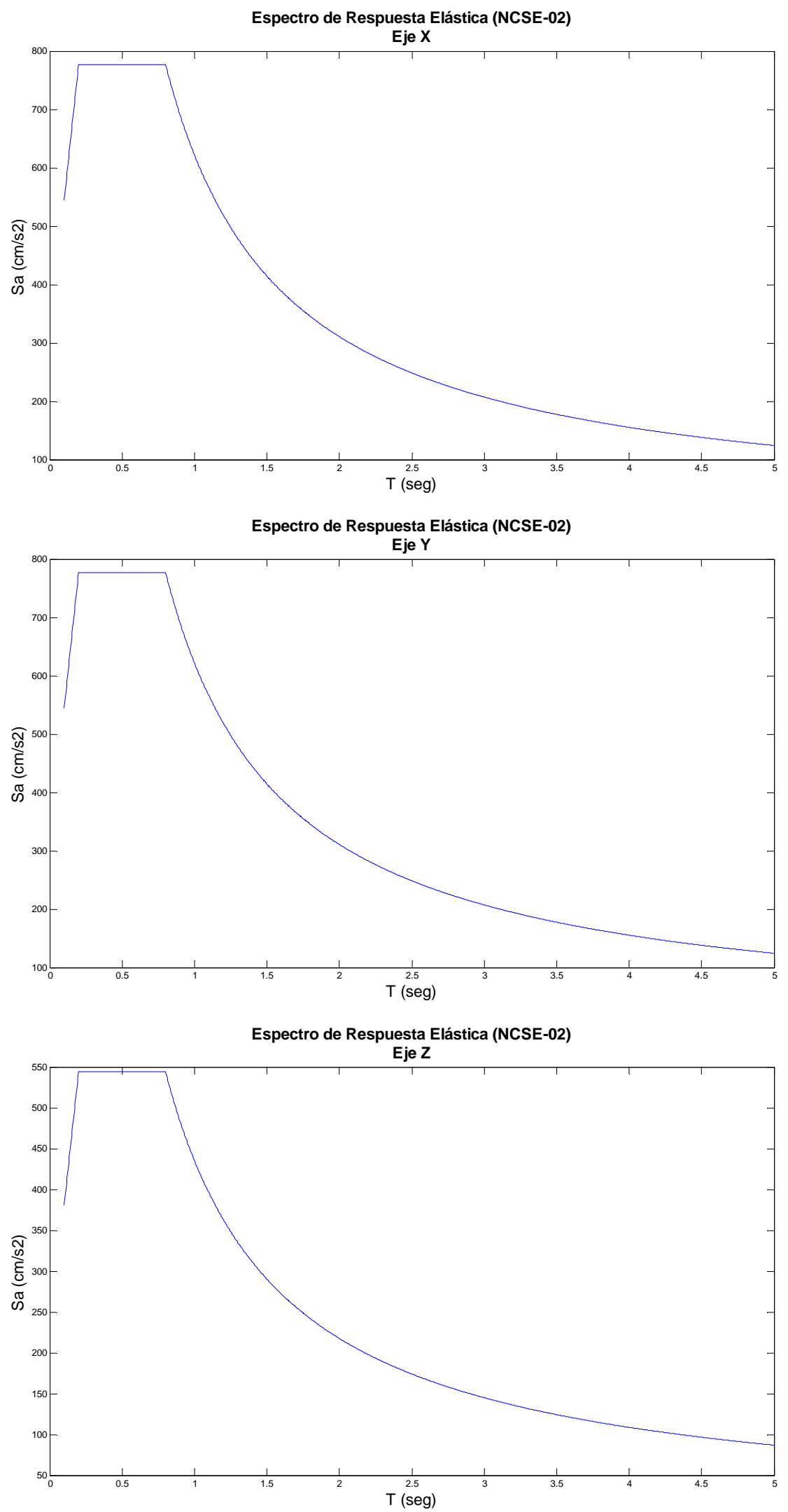

Figura 7.16. Espectros de respuesta elásticos de la normativa según los ejes 


\subsubsection{Simulación por el modelo estadístico:}

Usando el terremoto del 5 de Enero de 2007 de Sierra Elvira, que se puede ver en el Anejo 1, realizamos una simulación del modelo estadístico, con una magnitud momento esperada 5.0 y una frecuencia de esquina esperada de $0.5 \mathrm{~Hz}$, para un seísmo registrado en la un acelerógrafo ubicado en el cementerio de Santa Fé (Granada), el cual está catalogado como que se encuentra sobre rellenos, y da unas lecturas un tanto singulares. Estos nos hace pensar que se trata de un registro malo, además de estar situado en una zona de suelos blandos a muy blandos, por lo que tendrán que aparecer grandes amplificaciones de sitio.

De la simulación con el modelado estadístico obtenemos los siguientes resultados mostrados en la Tabla 7.6, Fig.7.17., Fig.7.18 y Fig.7.19.

Tabla 7.6. Datos de la simulación

\begin{tabular}{|c|c|c|c|}
\hline Datos del Medio & & & \\
\hline Velocidad de Ondas S & \multicolumn{2}{|r|}{3.5} & \\
\hline Velocidad de Ruptura & \multicolumn{2}{|c|}{2.975} & \\
\hline Datos de los Eventos & Evento EGF & Evento Objetivo & \\
\hline Magnitud Momento & 4.1 & 5 & \\
\hline Momento Sísmico & $1.58 \mathrm{E}+15$ & $3.55 \mathrm{E}+16$ & \\
\hline Frecuencia de Esquina & 1.4 & 0.5 & \\
\hline Caida de Esfuerzos & 8.6217 & 8.7926 & \\
\hline Factor C & \multicolumn{2}{|c|}{1.023} & \\
\hline Factor $\eta$ & \multicolumn{2}{|r|}{61} & \\
\hline Factor $\xi$ & \multicolumn{2}{|c|}{0.3654} & \\
\hline
\end{tabular}

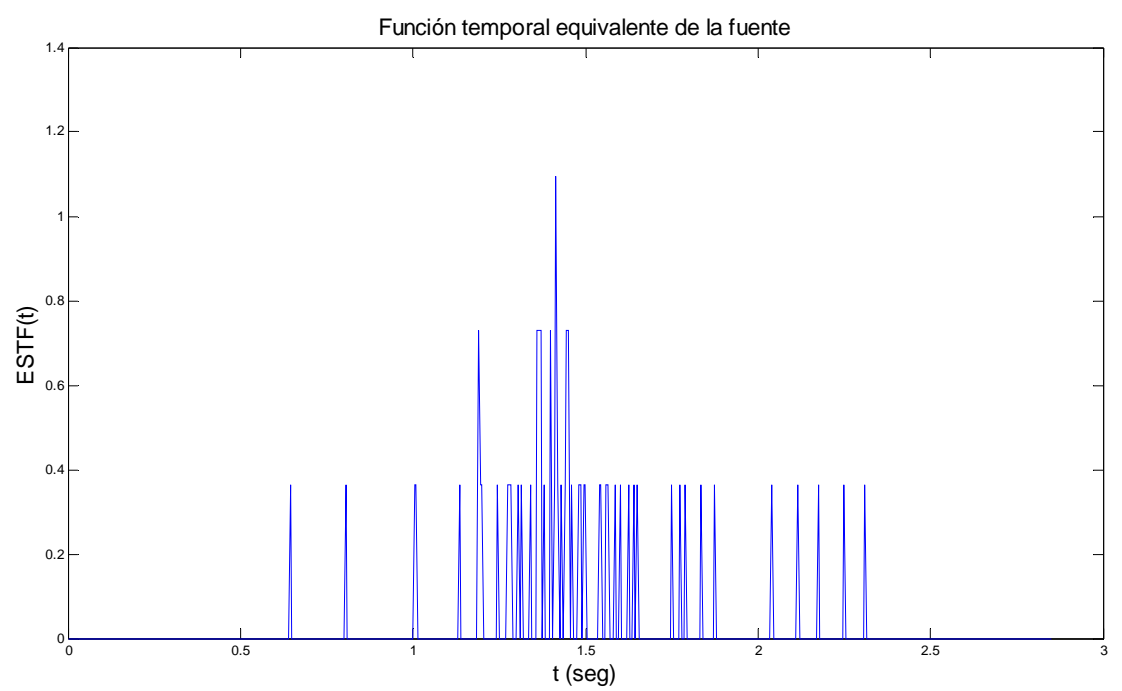

Figura 7.17. Función temporal equivalente de la fuente 

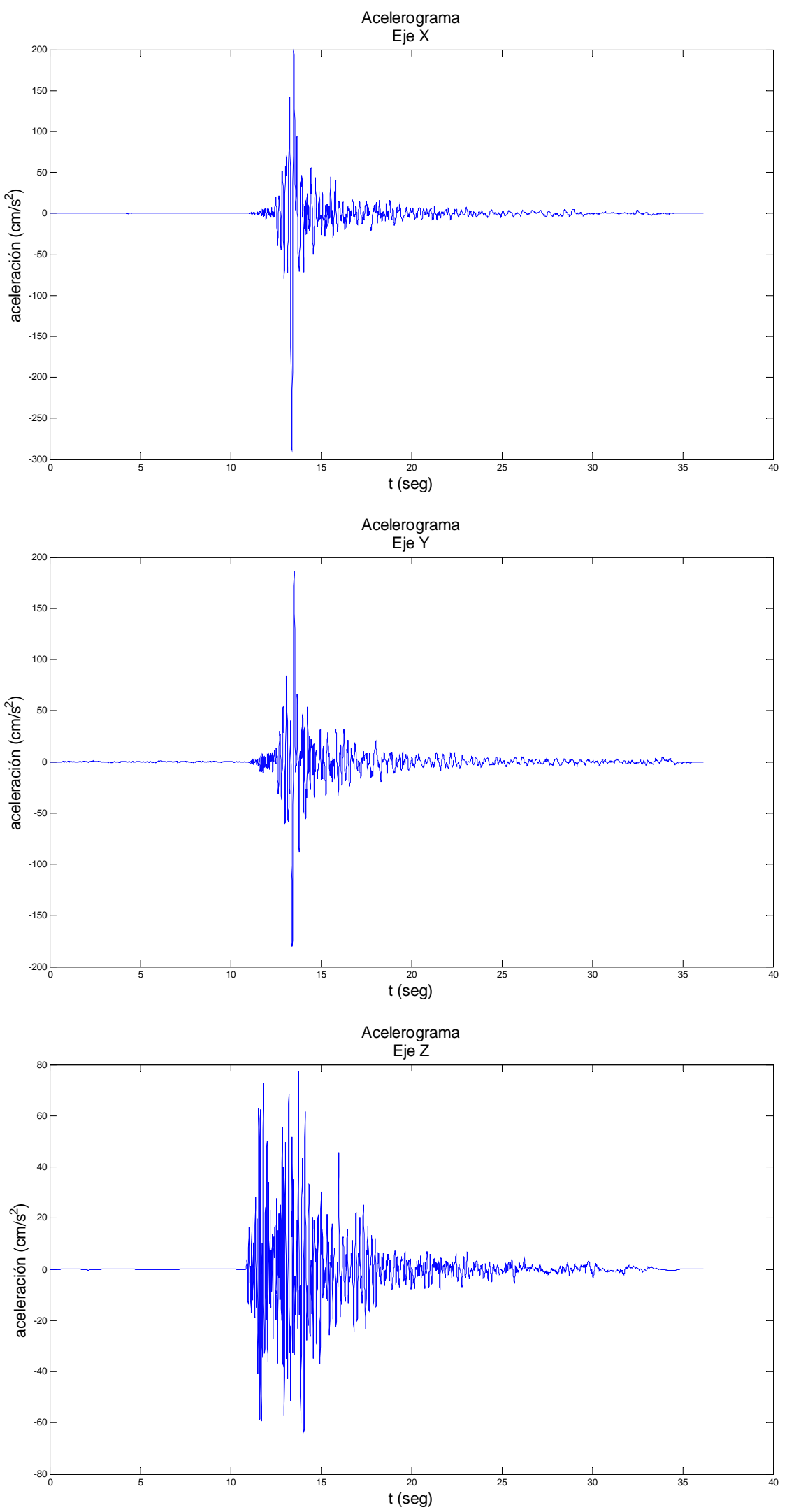

Figura 7.18. Acelerogramas simulados 

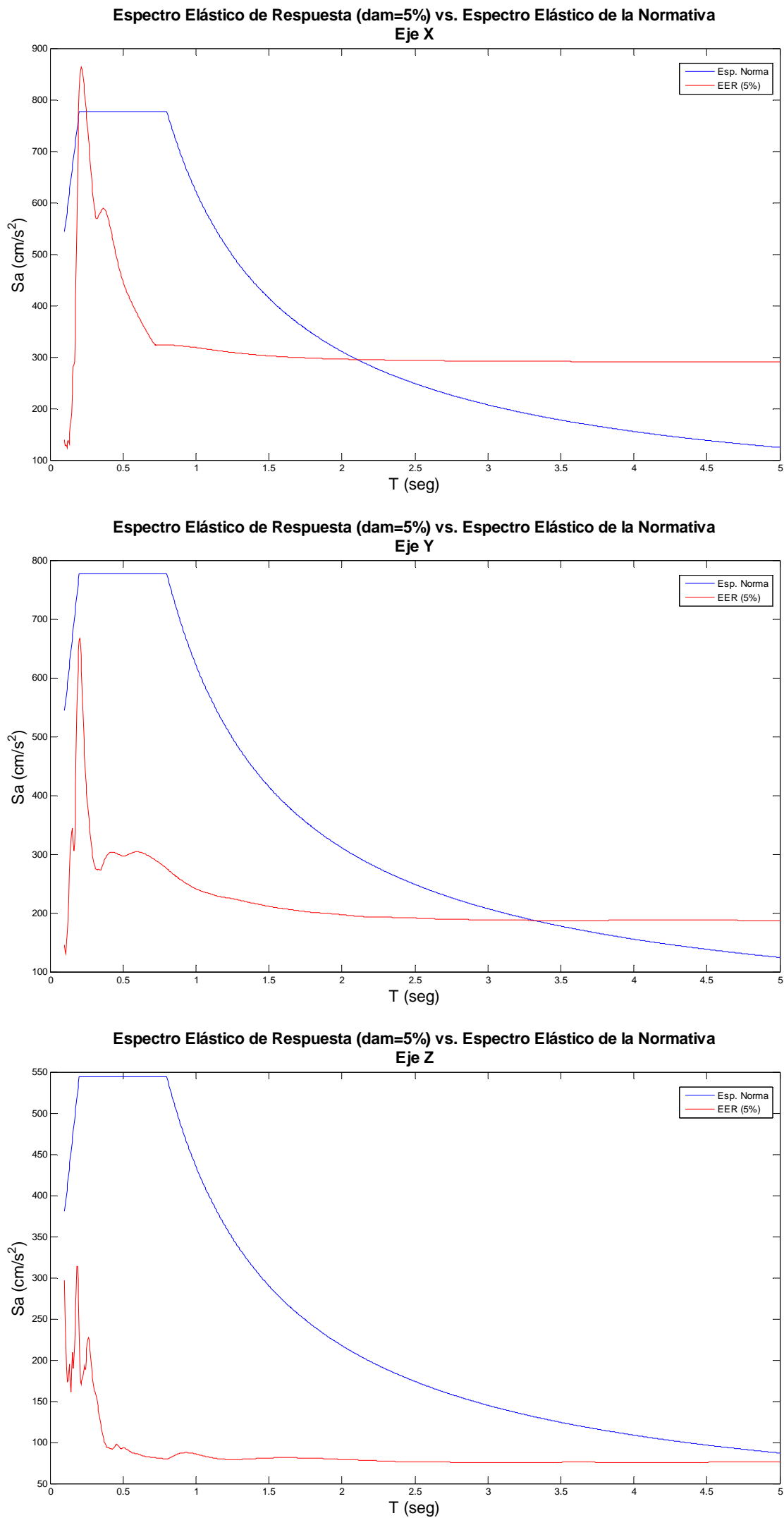

Figura 7.19. Espectros de evento simulado comparados con los espectros de la normativa 
Tanto en los acelerogramas simulados, como en los espectros, se ve un pulso que distorsiona la simulación con este modelo, además de ser un registro en suelo blando, que también procura grandes amplificaciones.

\subsubsection{Simulación por el modelo cinemático.}

Vistos los resultados de la simulación para el registro anterior de Santa Fé (Granada), vamos a realizar la misma simulación pero con el modelo cinemático, así podremos observar el comportamiento de cada uno de los métodos para el caso de un registro muy distorsionado.

Los resultados que obtenemos vienen dados por la tabla de datos de la simulación (Tabla 7.7.), la función temporal equivalente de a la fuente (Fig.7.20), los acelerogramas simulados (Fig.7.21.) y espectros comparados por cada eje (Fig.7.22.).

Tabla 7.7. Datos de la simulación

\begin{tabular}{|c|c|c|c|}
\hline Datos del Medio & & & \\
\hline Velocidad de Ondas S & \multicolumn{2}{|r|}{3.5} & \\
\hline Velocidad de Ruptura & \multicolumn{2}{|c|}{2.975} & \\
\hline Datos de los Eventos & Evento EGF & Evento Objetivo & \\
\hline Magnitud Momento & 4.1 & 5 & \\
\hline Momento Sísmico & $1.58 \mathrm{E}+15$ & $3.55 \mathrm{E}+16$ & \\
\hline Frecuencia de Esquina & 1.4 & 0.5 & \\
\hline Caida de Esfuerzos & 8.6217 & 8.7926 & \\
\hline Factor $\mathrm{N}$ & \multicolumn{2}{|r|}{3} & \\
\hline Factor C & \multicolumn{2}{|c|}{1.023} & \\
\hline
\end{tabular}

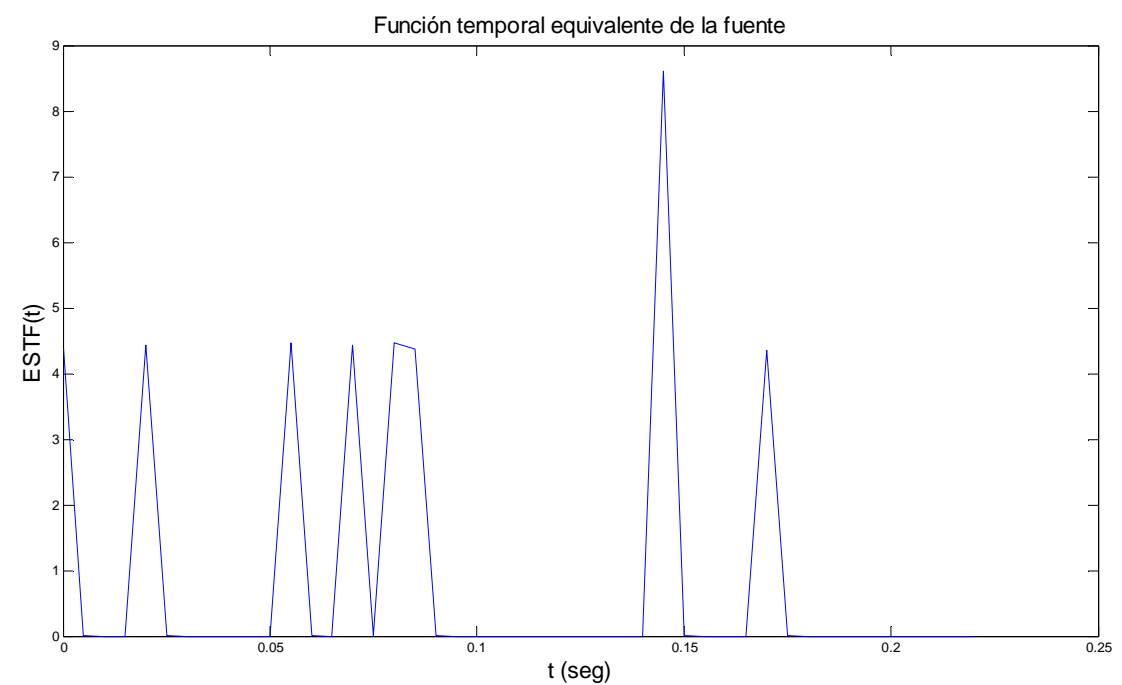

Figura 7.20. Función temporal equivalente de la fuente 

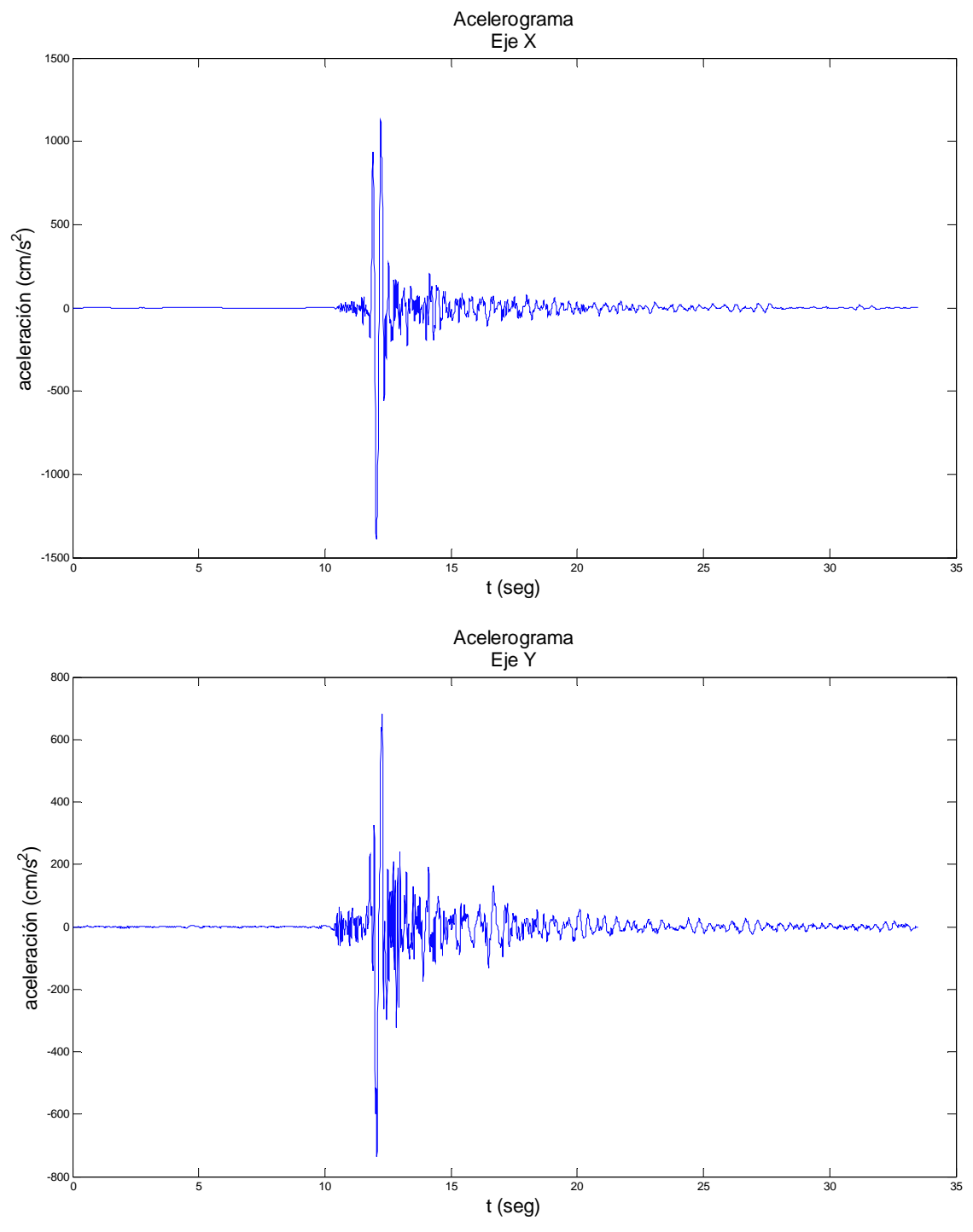

Acelerograma

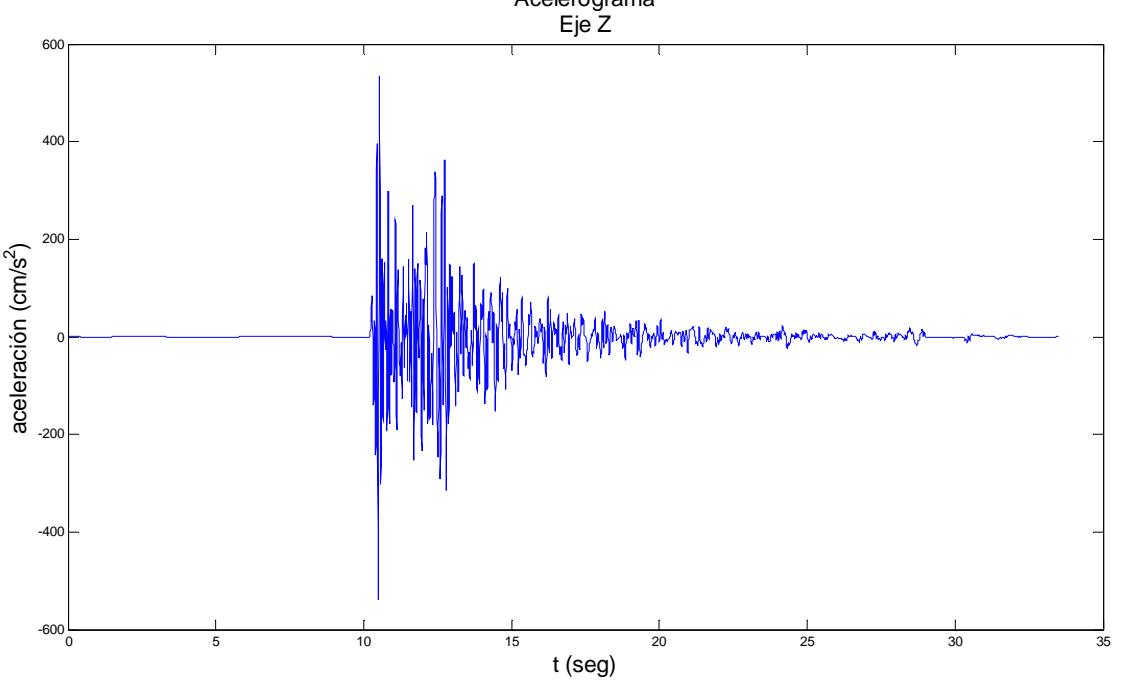

Figura 7.21. Acelerogramas simulados 

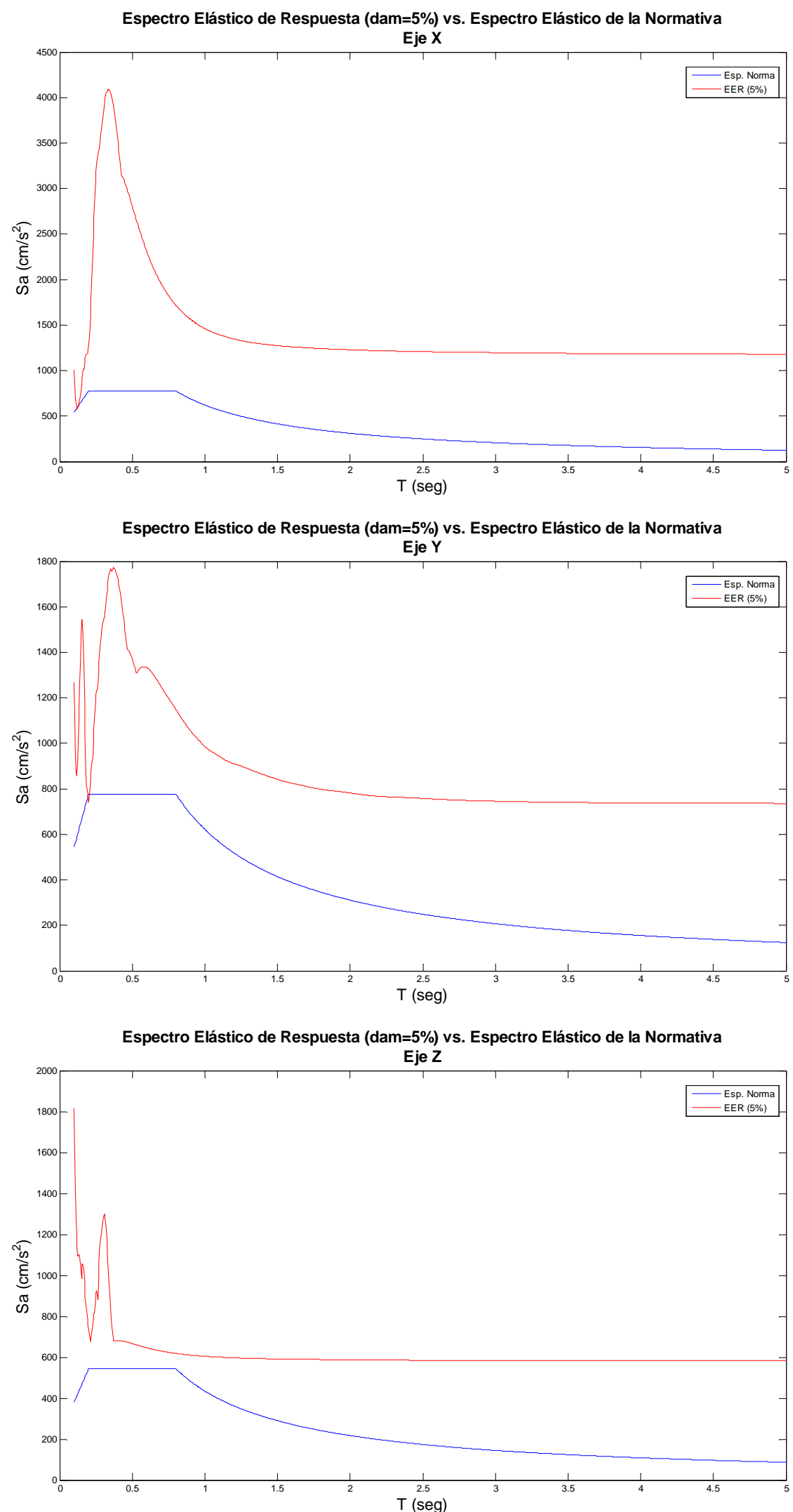

Figura 7.22. Espectros de evento simulado comparados con los espectros de la normativa 
Claramente se observa, que se trata de una simulación irreal, nada plausible, para una magnitud "tan baja", que ocurran aceleraciones de este tipo. Se trata de un registro malo, con un pulso que hace que todas las simulaciones se vean muy afectadas.

Que esta afección se note más en el modelo cinemático que en el modelo estadístico es por el número de subeventos que se utilizan para las respectivas simulaciones. Si se utilizan pocos, y "escalados" con un parámetro de rango igual a la unidad, la interferencia de un mal registro, como función de Green empírica, será mucho mayor, que si se usan mas subeventos "escalados" por un parámetro mucho menor que la unidad.

\subsection{Discusión sobre la aplicación práctica de los modelos:}

En este apartado se ha intentado poner un orden y rango a la hora de realizar las simulaciones, para que no se generen registros irreales poco útiles, y que incluso puedan llevar a error al aplicarlos al cálculo de estructuras.

El parámetro $C$, que relaciona la caída de esfuerzos del evento objetivo, con el evento EGF (función de Green empírica), tiene que ser siempre igual o superior a la unidad. Y además hay que tener en cuenta que a mayor $C$ las aceleraciones que vamos a encontrarnos son mucho mayores, por lo que hay que tener cuidado qué frecuencia de esquina se usa para la obtención del evento objetivo.

La relación entre las frecuencias de esquina EGF y objetivo, $N$ nos da el orden de subeventos que vamos a tener que usar en cada una de las simulaciones, $N^{2}$ para el modelo cinemático y $N^{4}$ para el modelo estadístico. Este número de subeventos luego están "escalados" por $C$ y por $\xi$, para el modelo cinemático y estadístico, respectivamente. Por tanto como $C$ siempre será mucho mayor que $\xi$ y en el modelado cinemático se utilizan muchos menos subeventos, este modelado se verá muy afectado ante registros EGF distorsionados, mientras que para el modelado estadístico al tener muchos más subeventos pero que contribuyen en menor medida por estar multiplicados por $\xi$, esta afección será mucho menor.

En líneas generales, para los dos tipos de modelados, se puede decir que las simulaciones que se pueden realizar serán tan buenas como bueno sea el registro usado como función de Green empírica. Ya que afecciones en este registro, distorsionarán en menor o mayor medida el registro simulado. 


\section{CONCLUSIONES Y FUTURAS LÍNEAS DE INVESTIGACIÓN.}

Del desarrollo de este Trabajo Fin de Máster podemos sacar las siguientes conclusiones:

- La acción sísmica representada en el dominio del tiempo es un complemento en la etapa de diseño de una estructura. Los espectros de respuesta de proyecto expuestos por la normativa pueden servir para el predimensionamiento de la estructura, al igual que los espectros de input de energía nos son muy necesarios para el diseño e inclusión de disipadores u otros elementos del proyecto sismorresistente. Los acelerogramas sintéticos que podemos obtener de los modelados desarrollados en los puntos anteriores nos ayudan a poner a prueba el diseño de la estructura.

- Para comprender correctamente la acción sísmica es necesario tener una base sólida de conceptos de la sismología como la magnitud momento, el momento sísmico, el tensor de momentos, el mecanismo focal, el teorema de representación, etc.

- El enfoque teórico a la simulación de movimientos intensos del terreno requiere de muchas simplificaciones y solo está desarrollado para modelos concretos, de ahí la clara ventaja que obtiene el enfoque semiempírico al usar registros de eventos menores para simular eventos mayores.

- En este trabajo se han desarrollado sendos códigos en matlab, aplicando las múltiples publicaciones de Ordaz, Singh y Arboleda y de Irikura et al., de los que hemos denominado método OSA y método de Irikura, respectivamente. Estos códigos son la herramienta para lograr los registros simulados que nos pusimos como objetivo principal.

- El modelado estadístico y en concreto el expuesto de Ordaz, Singh y Arboleda, requiere de pocos parámetros para obtener el evento objetivo, de hecho es el método más sencillo y rápido de entre los dos desarrollados en este trabajo. Solo basta fijar los valores objetivo, de magnitud momento y frecuencia de esquina, para realizar la simulación.

- El modelado cinemático desarrollado, el método de Irikura, por el contrario es algo más complicado de determinar los parámetros iniciales, como son el mecanismo focal y en particular el acimut y el buzamiento del plano de falla, o la determinación de una manera determinista del punto de inicio de la ruptura o hipocentro. Pero tiene la ventaja de poder simular terremotos con efectos de 
directividad, en los cuales la geometría y propagación de la ruptura tienen suma importancia, efecto muy relevante en campo cercano (caso del sismo de Lorca).

- En conjunto se han conseguido dos herramientas para obtener acelerogramas sintéticos, pero a la hora de ponerlos en práctica tenemos el problema de asemejarlos a los espectros elásticos de respuesta que aparecen en la normativa. Estos espectros vienen caracterizados por la aceleración básica de cálculo, el coeficiente de contribución y el coeficiente de los tipos de terreno contemplados en la normativa. Por tanto el obtener una relación entre estos espectros y los espectros elásticos de respuesta del acelerogramas simulado que se rigen por la magnitud momento y la frecuencia de esquina del evento objetivo, se ha de realizar a base de simulaciones reiterativas, cambiando los valores requeridos hasta encontrar un espectro que se asemeje. También la componente estocástica de los dos modelados, añade algo más de dificultad a la obtención de una simulación más semejante a los espectros de la normativa.

Como futuras líneas de investigación a desarrollar, se indican las siguientes:

- El desarrollar métodos semiempíricos que contemplen la dinámica de la ruptura a la hora de simular la función fuente equivalente.

- Calibrar los métodos desarrollados con mayor número de eventos, registrados en más estaciones, y a la vez refinar más la obtención de las frecuencias de esquina, mecanismos focales etc., para así conseguir unos resultados más exactos. Pudiendo realizar así simulaciones con claros efectos de directividad en la zona de ruptura.

- Ampliar el uso de los métodos desarrollados para poder modelar rupturas más complejas. Procedimientos que se harían usando varios eventos función de Green empírica para una misma simulación.

- Intentar aunar los modelados estadístico y cinemático para que la dispersión de tiempos de retardo quede restringida al subespacio formado por el plano de falla. 


\section{AGRADECIMIENTOS}

Este trabajo ha sido parcialmente realizado dentro del marco del proyecto CGL2011-30187-C0201-02, al cual muestro mi agradecimiento.

Al igual que al Instituto Andaluz de Geofísica y al Instituto Geográfico Nacional, por la información que me han proporcionado para la realización de este Trabajo Fin de Máster.

Mi más sincero agradecimiento a Gerardo Alguacil y Francisco Vidal por su tiempo y buena disposición para guiarme a lo largo de la realización de esta investigación.

Y también, mi agradecimiento a los compañeros y profesores del Máster Universitario de Estructuras del curso 2011-2012, por sus apoyos y colaboraciones a lo largo de todo este año. 


\section{REFERENCIAS}

AIJ. (1993). Earthquake motion and Ground conditions, The Architectural Institute of Japan (AIJ).

Aki, K., Richards P. G. (2002). Quantitative Seismology, second edition. University Science Books.

Akiyama, H. (1999). Metodología de proyecto sismorresistente de edificios basada en el balance energético. Editorial Reverté.

Bansal, B. K., Singh, S. K., Dharmaraju, R., Pacheco, J. F., Ordaz, M., Dattatrayam, R. S., Suresh, G. (2009). Source study of two small earthquakes of Delhi, India, and estimation of ground motion from future moderate local events, J. Seismol(2009) 13, 89-105.

Benavent-Climent, A. (2010). Estructuras Sismorresistentes, Ediciones Maia.

Birgören, G., Irikura, K. (2005). Estimation of Site Response in Time Domain Using the MeyerYamada Wavelet Analysis, Bull. Seism. Soc. Am. 95, 1447-1456.

Boore, D.M. (1983). Stochastic simulation of high-frequency ground motions based on seismological models of the radiated spectra, Bull. Seism. Soc. Am. 73, 1865-1894.

Bozorgnia, Y., Bertero, V. V. (2004). Earthquake Engineering from Engineering Seismology to Performance-Based Engineering, CRC Press.

Bykova, V. V., Aref'ev, S. S., Rivera, L. (2010). Simulation of Ground Motion in the Moscow Region Using the Empirical Green's Function, Physics of the Solid Earth, 2010, Vol. 46, No. 1, pp. 19-33.

Causse, M., Chaljub, E., Cotton, F., Cornou, C., Bard, P.Y. (2009). New approach for coupling k ${ }^{-2}$ and empirical Green's functions: application to the blind prediction of broad-band ground motion in the Grenoble basin, Geophys. J. Int. (2009) 179, 1627-1644.

Cervený, V. (2001). Seismic Ray Theory, Cambridge University Press.

Courboulex, F., Converset, J., Balestra, J., Delouis, B. (2010). Ground-Motion Simulations of the 2004 Mw 6.4 Les Saintes, Guadeloupe, Earthquake Using Ten Smaller Events, Bull. Seism. Soc. Am. 100, 116-180.

Courboulex, F., Larroque, C., Deschamps, A., Kohrs-Sansorny, C., Gélis, C., Got, J. L., Charreau, J., Stéphan, J. F., Béthoux, N., Virieux, J., Brunel, D., Maron, C. (2007). Seismic hazard on the French Riviera: observations, interpretations and simulations, Geophys. J. Int. (2007) 170, 387-400.

Courboulex, F., Virieus, J., Deschamps, A., Gibert, D., Zollo, A. (1996). Source investigation of a small event using empirical Green's functions and simulated annealing, Geophys. J. Int. 125, 768-780. 
Deichmann, N. (1999). Empirical Green's Functions: A comparison between pulse width measurements and deconvolution by spectral division, Bull. Seism. Soc. Am. 89, 178-186.

Elnashai, A. S., Di Sarno, L. (2008). Fundamentals of Earthquake Engineering, John Wiley \& Sons, Ltd.

Fischer, T. (2005). Modeling of multiple events using empirical Green's functions: method, application to swarm earthquakes and implications for their rupture propagation, Geophys. J. Int. (2005) 163, 991-1005.

Honoré, L., Courboulex, F., Souriau, A. (2011). Ground motion simulations of a major historical earthquake (1660) in the French Pyrenees using recent moderate size earthquakes, Geophys. J. Int. (2011) 187, 1001-1018.

Hough, S. E. (2001). Empirical Green's Function Analysis of Recent Moderate Events in California, Bull. Seism. Soc. Am. 93, 456-467.

Irikura K., Kagawa, T., Sekiguchi, H. (1997). Revision of the empirical Green's function method, B25 of "Programme and Abstracts", Seismological Sciety of Japan, Vol. 2. 1997 (in Japanese).

Irikura, K. (1983). Semi-empirical estimation of strong ground motions during large earthquakes, Bull. Disaster. Prevention. Res. Insti. (Kyoto University) 32, 63-104.

Irikura, K. (1986). Prediction of strong acceleration motions using empirical Green's function, Proceedings of the Seventh Japan Earthquake Engineering Simp. 26, 151-156.

Irikura, K., Kamae, K. (1994). Estimation of strong ground motion in broad-frequency band based on a seismic source scaling model and an empirical Green's function technique, Annali di Geofisica. Vol XXXVII, N.6, 1721-1743.

Jaimes, M. A., Reinoso, E., Ordaz, M. (2008). Empirical Green's Functions Modified by Attenuation for Sources Located at Intermediate and Far Distances from the Original Source, Journal of Earthquake Engineering 12, 584-595.

Jarpe, S. P., Kasameyer, P. W. (1996). Validation of a Procedure for Calculating Broadband Strong Motion Time Histories with Empirical Green's Functions, Bull. Seism. Soc. Am. 86, 1116-1129.

Kagawa, T., Irikura, K., Somerville, P. G. (2004). Diferences in ground motion and fault ruptura process between the surface and buried ruptura earthquakes, Earth Planets Space, 56, 3-14, 2004.

Kamae, K., Bard, P.Y., Irikura, K. (1998). Prediction of strong ground motion at EURO-SEISTEST site using the empirical Green's function method, Journal of Seismology2, 193-207, 1998.

Kamae, K., Irikura, K, Pitarka, A. (1998). A technique for simulating strong ground motion using Hybrid Green's Function, Bull. Seism. Soc. Am. 88, 357-367.

Kamae, K., Kawabe, H. (2004). Source model composed of asperities for the 2003 Tokachi-oki, Japan, earthquake $\left(\mathrm{M}_{\mathrm{JMA}}=8.0\right)$ estimated by the empirical Green's function method, Earth Planets Space, 56, 323-327.

Kanamori, H. (1979). A semi-empirical approach to prediction of long-period ground motions from great earthquakes, Bull. Seism. Soc. Am. 69, 1645-1670. 
Khojastchfar, E., Daryan A. S., Assarch, M. A. (2009). Probabilistic Empirical Green's Function Method in Ground Motion Simulation, American J. of Engineering and Applied Sciences 2 (1) 160, 160-164.

Midorikawa, S. (1993). Semi-empirical estimation of peak ground acceleration from large earthquakes, Tectonophysics. 218, 287-295.

Miyake, H., Iwata, T., Irikura, K. (2003). Source Characterization for Broadband Ground-Motion Simulation: Kinematic Heterogeneous Source Model and Strong Motion Generation Area, Bull. Seism. Soc. Am. 93, 2531-2545.

Miyake, H., Tanaka, Y., Sakaue, M, Koketsu, K, Ishigaki, Y. (2006). Empirical Green's function simulation of broadband ground motions on Genkai Island during the 2005 West Off Fukuoka Prefecture earthquake, Earth Planets Space, 58, 1637-1642.

Morales, J., Singh, S.K., Ordaz, M. (1996). Analysis of the Granada (Spain) earthquake of 24 June $1984(\mathrm{M}=5)$ with emphasis on seismic hazard in the Granada Basin, Tectonophysics. 257, 253-263.

Motazeidian, D., Atkinson, G. M. (2005). Stochastic Finite Fault Modeling Based on a Dynamic Corner Frequency, Bull. Seism. Soc. Am. 95, 995-1010.

Nozu, A., Uwabe, T. (2000). Applicability of Empirical Green's Function Method to strong motion records on Man-Made Island in Kobe, 12WCEE2000, 0538.

Ordaz, M., Arboleda, J., Singh S.K. (1995). A Scheme of Random Summation of an Empirical Green's Function to Estimate Ground Motions from Future Large Earthquakes, Bull. Seism. Soc. Am. 85, 1635-1647.

Pavic, R., Koller, M. G., Bard P. Y., Lacavc-Lachct, C. (2000). Ground motion prediction with the empirical Green's function technique: an assessment of uncertainties and confidence level, Journal of Seismology 4, 59-77, 2000.

Pitarka, A., Somerville, P., Fukushima, Y, Uetake, T., Irikura, K. (2000). Simulation of near-fault strong-ground motion using Hybrid Green's Functions, Bull. Seism. Soc. Am. 90, 566-586.

Poiata, N., Miyake, H. (2006). Broadband ground motion simulation of Romanian Earthquakes using empirical Green's Function Method, 1st ECEES 2006, paper no. 506.

Salichon, J., Kohrs-Sansorny, C., Bertrand, E., Courboulex, F. (2010). A Mw 6.3 earthquake scenario in the city of Nice (sourtheast France): ground motion simulation, J. Seismol(2010) 14, 523-541.

Sanz de Galdeano, C., Peláez Montilla, J.A., López Casado, C. (2003). Seismic potenctial of the main active faults in the Granada Basin (Southern Spain), Pure appl. Geophys. 160 (2003) 1537-1556.

Shearer, P. M. (1999). Introduction to Seismology, second edition, Cambridge University Press. Shomali, Z.H. (2001). Empirical Green's functions calculated from the inversion of earthquake radiation patterns, Geophys. J. Int. (2001) 144, 647-655.

Sonley, E., Atkinson G.M. (2001). Apparent source spectra for earthquakes in the Charlevoix Seismic Zone: a comparison of Direct and Empirical Green's Function Methods, Bull. Seism. Soc. Am. 91, 1729-1740. 
Vallée, M. (2004). Stabilizing the Empirical Green Function Analysis: Development of the Projected Landweber Method, Bull. Seism. Soc. Am. 94, 394-409.

Velasco, A. A., Ammon, C. J., Lay, T. (1994). Empirical Green function deconvolution of broadband surface waves: Rupture directivity of the 1992 Landers, California (Mw=7.3), earthquake, Bull. Seism. Soc. Am. 84, 735-750.

Wennergerg, L. (1990). Stochastic summation of empirical Green's functions, Bull. Seism. Soc. Am. 80, 1418-1432.

Wössner, J., Treml, M, Wenzel, F. (2002). Simulation of Mw=6.0 earthquakes in the Upper Rhinegraben using empirical Green functions, Geophys. J. Int. (2002) 151, 487-500. 


\section{ANEJO 1: CATALOGO DE TERREMOTOS UTILIZADOS PARA LAS SIMULACIONES}

En este apartado se muestran los datos y acelerogramas de los terremotos usados a lo largo de todo el documento.

\section{Terremoto del 24 de Febrero de 1997, Agrón}

$\begin{array}{llr}\text { Evento } & \text { Agrón } & \\ & \text { Fecha: } & \text { 24/02/1997 } \\ & \text { Hora: } & 7: 09: 50\end{array}$

\begin{tabular}{|l|c|c|r|l|}
\hline$\underline{\text { Coordenadas }}$ & $\mathrm{X}$ & $\mathrm{Y}$ & \multicolumn{1}{c|}{$\mathrm{Z}$} & (m) \\
\cline { 1 - 4 } Hipocentro & 425815 & 4098148 & -16000 & ED50 \\
\hline$\underline{\text { Estación 1 }}$ & 447145 & 4116199 & 775 & CRT \\
\hline & & & \\
\hline
\end{tabular}

Huso 30

Terr. Tipo I

Mecanismo Focal

\begin{tabular}{|c|c|}
\hline Acimut & 300 \\
\hline Buzamiento & 80 \\
\hline Rake & -90 \\
\hline
\end{tabular}

\begin{tabular}{|l|r|r|}
\hline Magnitud Momento & 4.3 \\
\hline Momemento sísmico & & $2.94 \mathrm{E}+15$ \\
\hline Frecuencia de & $\mathrm{N}-\mathrm{m}$ \\
Esquina & 1.2 & $\mathrm{~Hz}$ \\
\hline Caida de Esfuerzos & 11 & $\mathrm{bar}$ \\
\hline
\end{tabular}

\begin{tabular}{|l|r|l}
\hline Velocidad de las ondas S & 3.5 & $\mathrm{Km} / \mathrm{s}$ \\
\hline Densidad & 3.2 & $\mathrm{gr} / \mathrm{cm} 3$
\end{tabular}

Frecuencia de muestreo:

250

Registro en la Estación 1: Observatorio de Cartuja (Granada): 

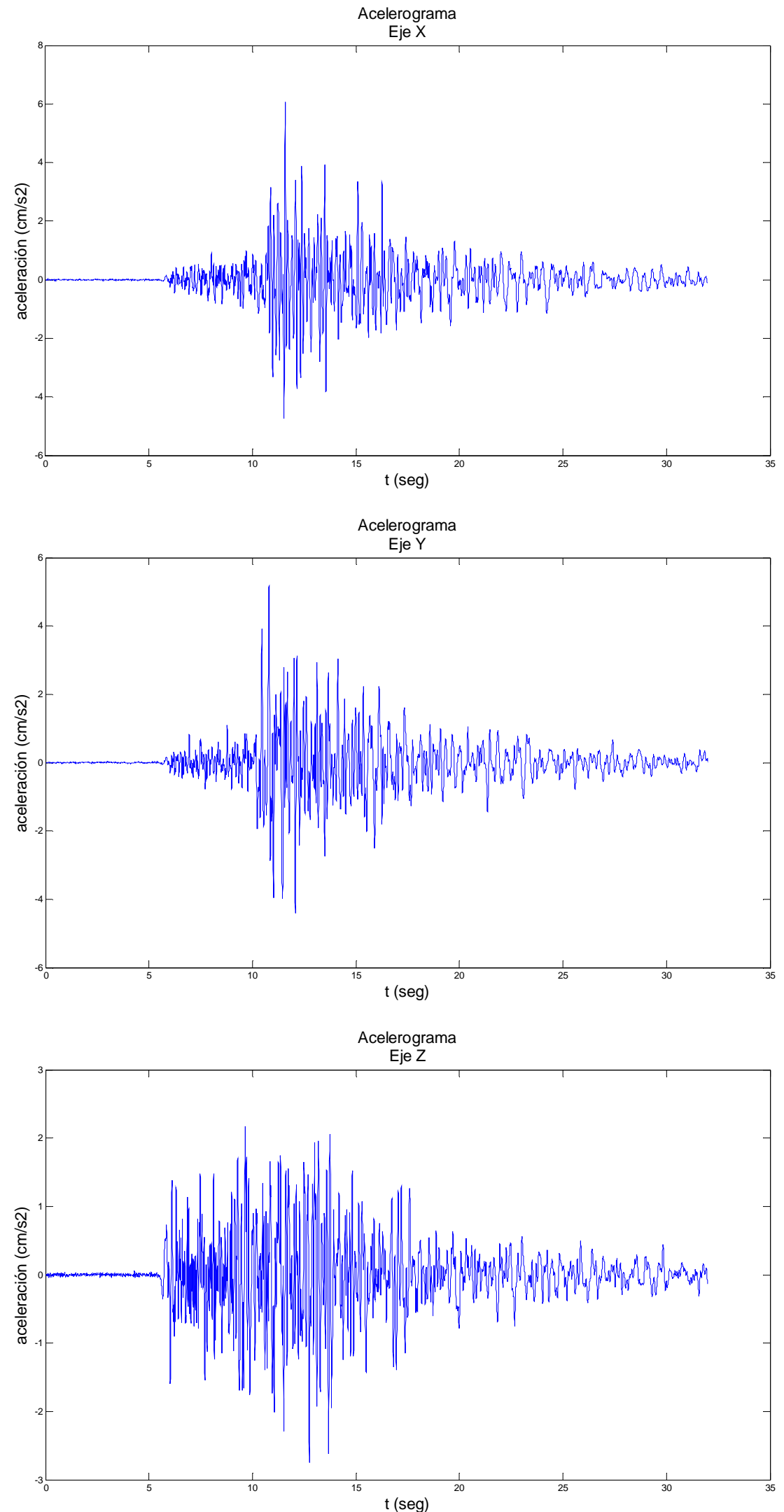
Terremoto del 5 de Enero de 1997, Sierra Elvira

$\begin{array}{llr} & \text { Sierra } & \\ \text { Evento } & \text { Elvira } & \\ & \text { Fecha: } & 05 / 01 / 2007 \\ & \text { Hora: } & 0: 32: 32\end{array}$

\begin{tabular}{|l|c|c|rlll}
\hline Coordenadas & X & Y & \multicolumn{2}{|l}{ Z } & (m) & \\
\cline { 1 - 4 } Hipocentro & 431832.294 & 4116182.59 & -1000 & ED50 & Huso 30 \\
\cline { 1 - 3 } Estación 1 & 445942.056 & 4115075.73 & 666 & FaculCC & Terr.Tipo III \\
\cline { 1 - 4 } Estación 2 & 437125.398 & 4115840.58 & 585 & Cem.S.Fé & Terr.Tipo IV
\end{tabular}

Mecanismo Focal

\begin{tabular}{|c|c|}
\hline Acimut & 90 \\
\hline Buzamiento & 45 \\
\hline Rake & \\
\hline
\end{tabular}

\begin{tabular}{|l|r|r|}
\hline Magnitud Momento & 4.1 \\
\hline$\underline{\text { Momemento sísmico }}$ & $1.58 \mathrm{E}+15$ & N-m \\
\hline Frecuencia de & 1.4 & $\mathrm{~Hz}$ \\
\hline$\underline{\text { Esquina }}$ & 8.6217 & bar \\
\hline$\underline{\text { Caida de Esfuerzos }}$ & $\underline{y}$ &
\end{tabular}

\begin{tabular}{|l|r|r|}
\hline Velocidad de las ondas S & 3.5 & $\mathrm{Km} / \mathrm{s}$ \\
\cline { 1 - 2 } Densidad & 3.2 & $\mathrm{gr} / \mathrm{cm} 3$
\end{tabular}

Frecuencia de muestreo:

200

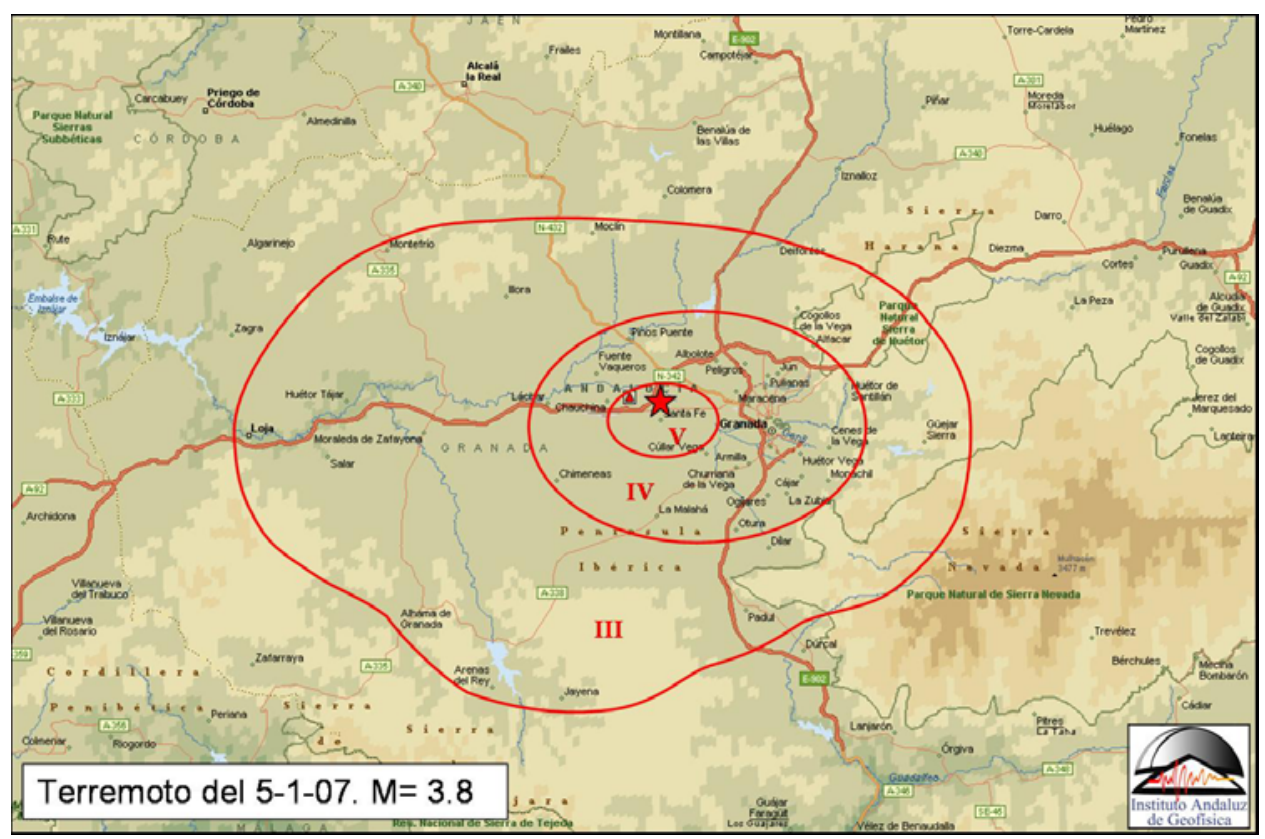


Registro en la Estación 1: Facultad de Ciencias de la Universidad de Granada: Acelerograma

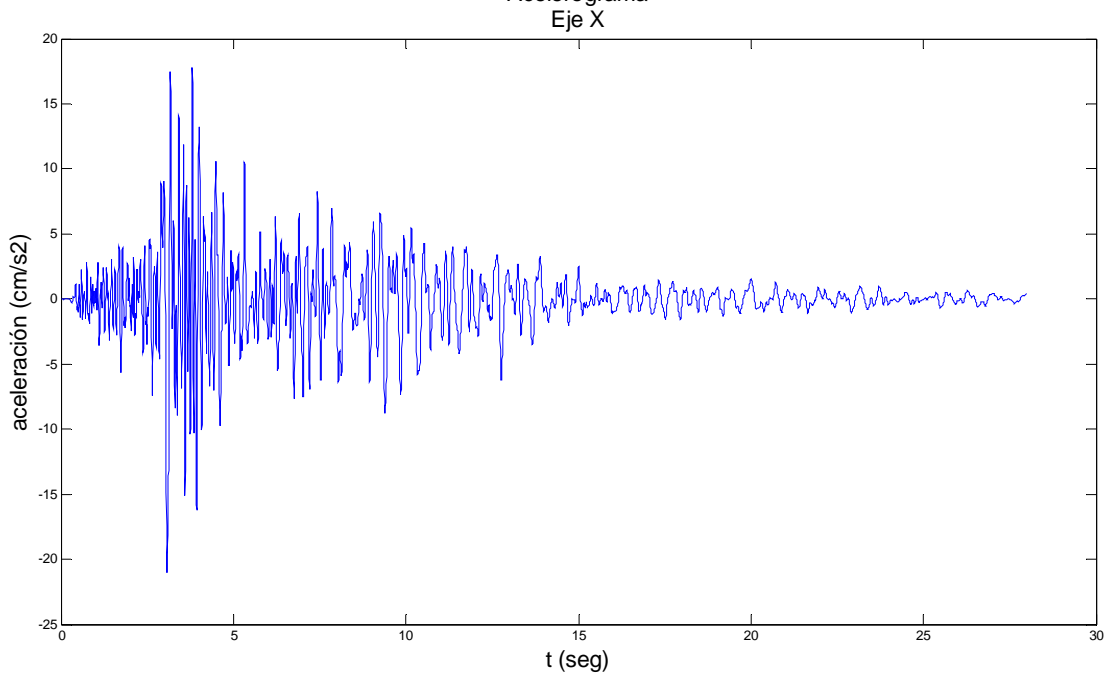

Acelerograma

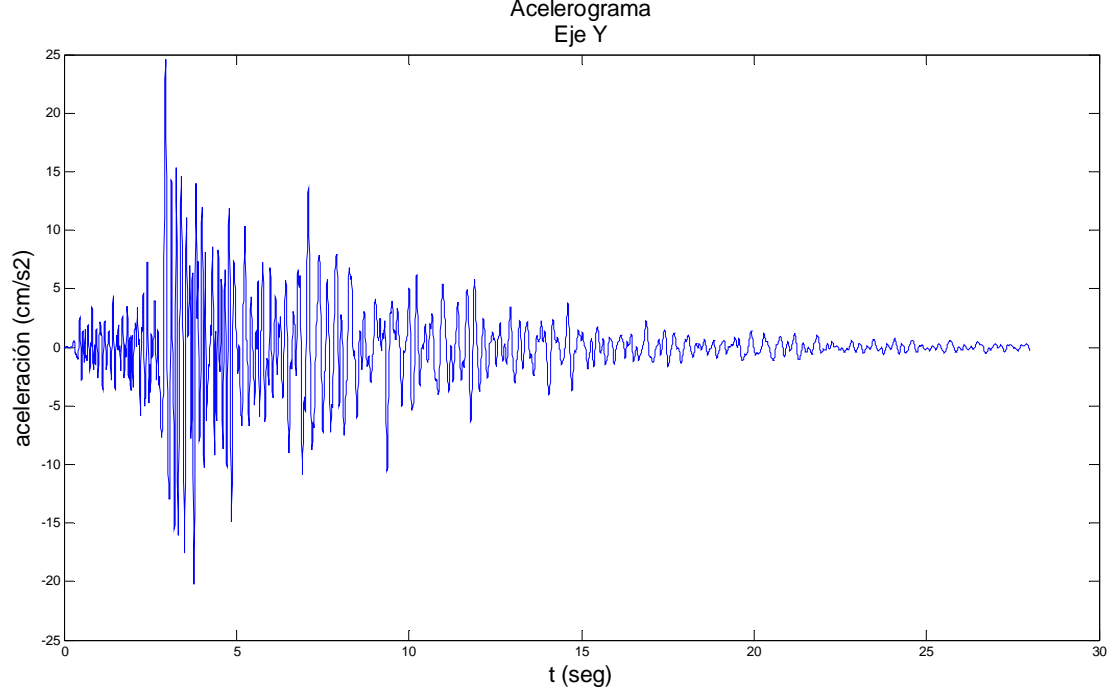

Acelerograma

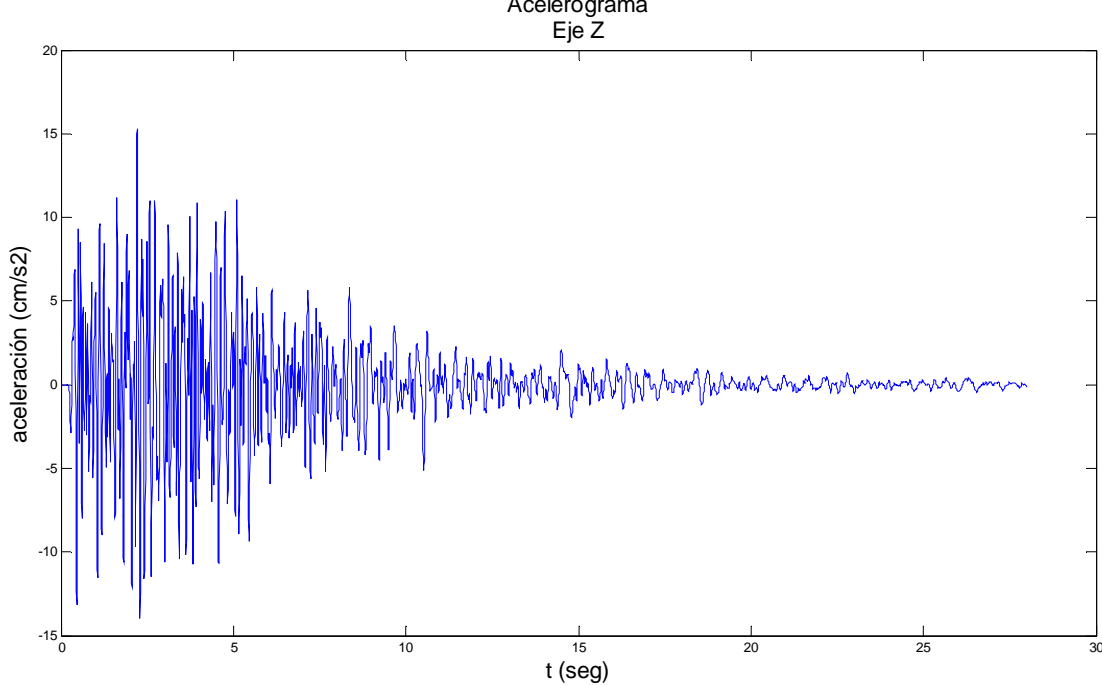


Registro en la Estación 2: Cementerio de Santa Fé:
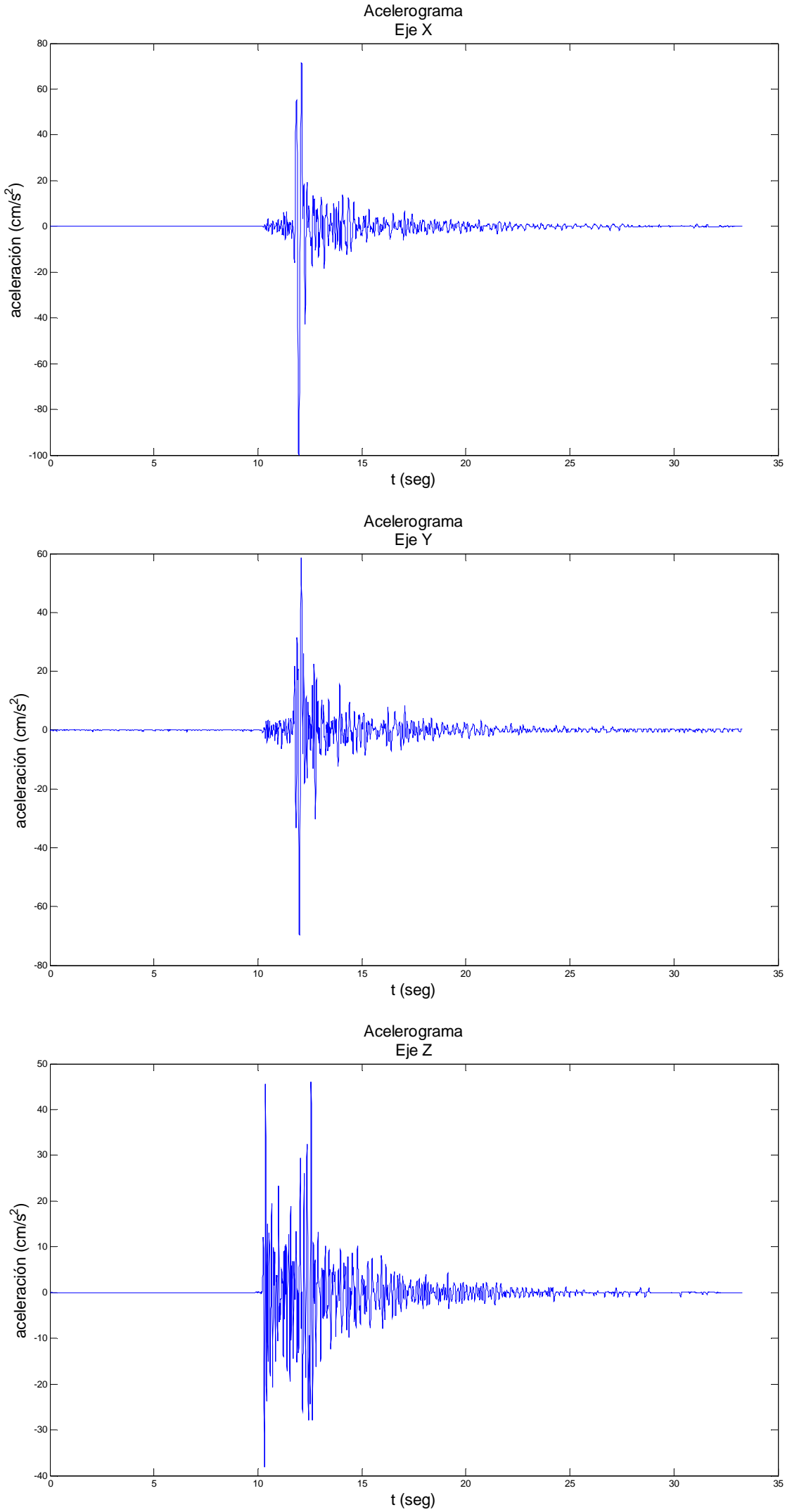
Autor:

Ing. Francisco Antonio García Villena

Tutores:

Dr. Gerardo Alguacil de la Blanca

Dr. Francisco Vidal Sánchez 
